\title{
Lie Groups of Fourier Integral Operators on Open Manifolds
}

\author{
JÜRgEN EICHHORN AND RUdOLF SCHMID ${ }^{1}$
}

\begin{abstract}
We endow the group of invertible Fourier integral operators on an open manifold with the structure of an ILH Lie group by endowing the invertible pseudodifferential operators and the contact transformations on an open manifold of bounded geometry with an ILH Lie group structure.
\end{abstract}

\section{Introduction.}

For finite dimensional Hamiltonian systems, the embedding as a coadjoint orbit is a very well known and convenient method for integration, at least for the construction of integrals. The same is valid in the infinite dimensional case. But, as in the finite dimensional case, the main problem is to find appropriate Lie groups such that the Hamiltonian system can be embedded as a coadjoint orbit. There are not many such candidates. For example, consideration of completed diffeomorphism groups leads to the following complications. First, they have a good Hilbert manifold structure but left multiplication and forming the inverse are only continuous operations, i.e., they don't have a Lie group structure. Secondly, considering the tangent space at the identity as a candidate for a Lie algebra causes additional difficulties. Namely, forming the Lie bracket decreases the Sobolev order, i.e., it is not a closed operation. One way out of this difficulty has been indicated by Omori [17], forming the inverse limit of such groups and algebras, labeled by the Sobolev index. In the compact case, this is an old and very well known story. In the open case, there arise tremendous difficulties which have been essentially overcome e.g., in [10], [15]. There are not many other candidates for infinite-dimensional Lie groups. Another very important class are the invertible pseudodifferential operators $\Psi D O$ s and Fourier integral operators FIOs on a manifold where invertibility means invertibility within this class. In the compact case, this has been established in [1], [2] and have been

\footnotetext{
${ }^{1}$ Research supported by the Emory-Greifswald Exchange Program.
} 
applied by Adler in [4] and Adams, Ratiu and Schmid [3] to the complete integrability of the $\mathrm{KdV}$ equation. Adler followed a suggestion of J. Moser and constructed an infinite sequence of integrals, tacitly assuming the Lie group structure of invertible FIOs on $S^{1}$. In the open case, there has not yet been any approach until today and we present here the complete solution of the topological side of the problem. The details are rather long and complicated. Hence we sketch here the main ideas and constructions and connect them with surrounding problems. The paper is organized as follows. In Section 2 we recall the for us main important facts concerning Sobolev spaces on open manifolds and completed diffeomorphism groups. Section 3 is devoted to open contact manifolds with bounded contact form $\theta$ associated to a metric $g$ of bounded geometry satisfying the spectral assumption

$$
\inf \sigma_{e}\left(\left.\Delta_{1}\right|_{\left(\operatorname{ker} \Delta_{1}\right)^{\perp}}\right)>0
$$

Here $\Delta_{1}$ means the Laplace operator on 1-forms and $\sigma_{e}$ denotes the essential spectrum. We prove that the identity component $\mathcal{D}_{\theta, 0}^{r+1}$ of the completed group of contact transformations is a Hilbert manifold and a topological group, $f \in \mathcal{D}_{\theta}^{r+1}$ if and only if $f^{*} \theta=\alpha \cdot \theta$ and $f$ is a Sobolev diffeomorphism. If additionally the metric $g$ has bounded geometry of infinite order then $\mathcal{D}_{\theta, 0}^{\infty}=\lim _{\leftarrow} \mathcal{D}_{\theta, 0}^{r}$ is an ILH Lie group. Here an ILH Lie group is roughly speaking the inverse $\operatorname{limit} \underset{\leftarrow}{\lim } G^{r}$ where $G^{r}$ is a topological group and Hilbert manifold modeled over a Sobolev space of index $r$. The exact definition will be given in section 3. Moreover, we introduce the group $\mathcal{D}_{\theta, e, 0}^{r+1}$ of restricted contact transformations, $f \in \mathcal{D}_{\theta, e, 0}^{r+1}$ if and only if $f^{*} \theta=\theta$ and $f$ is a Sobolev diffeomorphism belonging to the identity component. In Section 4 we apply these constructions to the restricted cotangent bundle $T^{*} M \backslash 0$ and the cosphere bundle $S\left(T^{*} M\right)$. Then the canonical 1-form $\theta$ on $S\left(T^{*} M\right)$ is a contact form associated to the Sasaki metric $g_{S} . \mathcal{D}_{\theta, 0}^{r+1}(S)$ is well defined, and we define $\mathcal{D}_{\theta, 0}^{r+1}\left(T^{*} M \backslash 0\right)$ by homogeneous of degree one extension. At the end we give several isomorphic descriptions of the tangent space $T_{i d} \mathcal{D}_{\theta, 0}^{r+1}\left(T^{*} M \backslash 0\right)$. Section 5 is devoted to the general notions and theorems concerning uniform $\Psi$ DO's and FIO's on open manifolds of bounded geometry. We restrict to FIO's whose homogeneous canonical relation is the graph $\Gamma(f), f \in \mathcal{D}_{\theta, 0}^{r+1}(S)$ and write $\mathcal{U} F^{q}(f)$ for this set. Denote $\mathcal{U} F^{q}(r+1)=\bigcup_{f \in \mathcal{D}_{\theta, 0}^{r+1}} \mathcal{U} F^{q}(f)$ and $\mathcal{U} F^{q, k}(r+1)=\mathcal{U} F^{q}(r+1) / \mathcal{U} F^{-k-1}(r+1)$. Similarly we have spaces $\mathcal{U} F^{q, k}(\infty)$ based on $\mathcal{D}_{\theta, 0}^{\infty}\left(T^{*} M \backslash 0\right)$. Denote by ()$_{*}$ the group of invertible elements. Then we construct a local section $\sigma$ of the 
exact sequence

$$
I \longrightarrow\left(\mathcal{U} \Psi^{0, k}\right)_{*} \stackrel{j}{\longrightarrow}\left(\mathcal{U} F^{0, k}\right)_{*} \stackrel{\pi}{\longrightarrow} \mathcal{D}_{\theta, 0}^{\infty} \longrightarrow e .
$$

Here $j$ is the inclusion and $\pi$ the map ( $\Gamma$ with canonical relation $\Gamma(f)) \rightarrow f$. This section is given by (5.60). Using certain Sobolev uniform structures, we obtain in section 6 Hilbert Lie groups $\left(\mathcal{U} \Psi^{0, k, s}\right)_{*}$. Then $\left(\mathcal{U} \Psi^{0, k}\right)_{*}=$ $\lim _{\leftarrow}\left(\mathcal{U} \Psi^{0, k, s}\right)_{*}$ has the structure of an ILH Lie group. Hence the outer terms in $(E S)$ have such a structure. We use these, the local section $\sigma$ and several group theoretic constructions to establish in section 7 an ILH Lie group structure for $\left(\mathcal{U} F^{0, k}\right)_{*}$.

The ILH Lie algebras are quite different from those in the compact case.

The main result of the paper is theorem 7.12. A remarkable feature of many geometric analytic investigations on open manifolds is the fact that we get interesting, meaningful results if we assume two conditions: bounded geometry and the spectral condition $\inf \sigma_{e}\left(\left.\Delta_{1}\right|_{\left(\operatorname{ker} \Delta_{1}\right)^{\perp}}\right)>0$. Our space $\left(\mathcal{U} F^{0, k}\right)_{*}$ is now the right group to embed infinite dimensional Hamiltonian systems and to construct an infinite sequence of integrals, e.g., for the KdV equation on the real line.

In a forthcoming paper, we extend the construction performed here to the group of all invertible Fourier integral operators and establish a KostantSymes theorem for the open case.

\section{Bounded Geometry and Sobolev Diffeomorphism Groups.}

We give a short summary of the basic facts. For details and proofs we refer to [9], [10], [12]. Let $\left(M^{n}, g\right)$ be an open Riemannian manifold. Consider the following conditions $(I)$ and $\left(B_{k}\right)$.

$$
\begin{aligned}
& r_{i n j}(M, g)=\inf _{x \in M} r_{i n j}(x)>0, \\
& \left|\nabla^{i} R\right| \leq C_{i}, \quad 0 \leq i \leq k,
\end{aligned}
$$

where $r_{i n j}$ denotes the injectivity radius and $R$ the curvature. We say $\left(M^{n}, g\right)$ has bounded geometry up to order $k$ if it satisfies $(I)$ and $\left(B_{k}\right)$.

Lemma 2.1. The condition (I) implies completeness.

Let $0 \leq k \leq \infty$ and $M^{n}$ be open. Then there always exists a metric $g$ satisfying $(I)$ and $\left(B_{k}\right)$, i. e. there is no topological obstruction against metrics of bounded geometry of any order. 
Let $T_{v}^{u}$ be the bundle of $u$-fold covariant and $v$-fold contravariant tensors and define

$$
\begin{aligned}
\Omega_{r}^{p}\left(T_{v}^{u}, g\right) & =\left\{\left.t \in C^{\infty}\left(T_{v}^{u}\right)|| t\right|_{g, p, r}:=\right. \\
& \left.=\left(\int \sum_{i=0}^{r} \mid \nabla^{i} t_{g, x}^{p} \operatorname{dvol}_{x}(g)\right)^{\frac{1}{p}}<\infty\right\} .
\end{aligned}
$$

Let $\bar{\Omega}^{p, r}\left(T_{v}^{u}, g\right)$ be the completion of $\Omega_{r}^{p}\left(T_{v}^{u}, g\right)$ with respect to ||$_{g, p, r}$, $\stackrel{\circ}{\Omega}^{p, r}\left(T_{v}^{u}, g\right)$ the completion of $C_{c}^{\infty}\left(T_{v}^{u}\right)$ with respect to ||$_{g, p, r}$ and $\Omega^{p, r}\left(T_{v}^{u}, g\right)$ the space of all distributional tensor fields $t$ with $|t|_{g, p, r}<\infty$. Then we have

$$
\stackrel{\circ}{\Omega}^{p, r}\left(T_{v}^{u}, g\right) \subseteq \bar{\Omega}^{p, r}\left(T_{v}^{u}, g\right) \subseteq \Omega^{p, r}\left(T_{v}^{u}, g\right) .
$$

Lemma 2.2. If $g$ satisfies $(I),\left(B_{k}\right)$, then

$$
\stackrel{\circ}{\Omega}^{p, r}\left(T_{v}^{u}, g\right)=\bar{\Omega}^{p, r}\left(T_{v}^{u}, g\right)=\Omega^{p, r}\left(T_{v}^{u}, g\right), \quad 0 \leq r \leq k+2 .
$$

Define

$$
\begin{aligned}
{ }^{b, m}|t|_{g} & :=\sum_{i=0}^{m} \sup _{x \in M}\left|\nabla^{i} t\right|_{g, x}, \\
{ }_{m}^{b} \Omega\left(T_{v}^{u}, g\right) & :=\left\{\left.t \in C^{\infty}\left(T_{v}^{u}\right)\left|{ }^{b, m}\right| t\right|_{g}<\infty\right\},
\end{aligned}
$$

${ }^{b, m} \Omega\left(T_{v}^{u}, g\right)$ the completion of ${ }_{m}^{b} \Omega\left(T_{v}^{u}, g\right)$ with respect to ${ }^{b, m}||_{g}$ and ${ }_{b, m} \stackrel{\circ}{\Omega}\left(T_{v}^{u}, g\right)$ the completion of $C_{c}^{\infty}\left(T_{v}^{u}\right)$ with respect to ${ }^{b, m}||_{g}$. Then ${ }^{b, m} \Omega\left(T_{v}^{u}, g\right)=\left\{t \mid t\right.$ is a $C^{m}$ tensor field and $\left.{ }^{b, m}|t|_{g}<\infty\right\}$.

If $(E, h, \nabla) \rightarrow\left(M^{n}, g\right)$ is a Riemannian vector bundle over $\left(M^{n}, g\right)$ with metric connection $\nabla$ then we make the analogous definitions, e. g. for $s \in C_{c}^{\infty}(E)$

$$
|s|_{p, r}:=\left(\int \sum_{i=0}^{r}\left|\nabla^{i} s\right|_{x}^{p} d v o l_{x}(g)\right)^{\frac{1}{p}}
$$

and obtain analogous spaces

$$
\begin{gathered}
\stackrel{\circ}{\Omega}^{p, r}(E, g, h, \nabla), \quad \bar{\Omega}^{p, r}(E, g, h, \nabla), \quad \Omega^{p, r}(E, g, h, \nabla), \\
{ }^{b, m} \stackrel{\circ}{\Omega}(E, g, h, \nabla), \quad{ }^{b, m} \Omega(E, g, h, \nabla) .
\end{gathered}
$$


For $(E, h, \nabla)$ there is an analogous condition $\left(B_{k}(E, \nabla)\right)$ as for $\left(B_{k}(M, g)\right)$,

$$
\left(B_{k}(E, \nabla)\right) \quad\left|\nabla^{i} R^{E}\right| \leq C_{i}, \quad 0 \leq i \leq k,
$$

where $R^{E}$ denotes the curvature of $(E, \nabla)$. The lemma 2.2 remains true correspondingly,

$(2.1) \stackrel{\circ}{\Omega}^{p, r}(E, g, h, \nabla)=\bar{\Omega}^{p, r}(E, g, h, \nabla)=\Omega^{p, r}(E, g, h, \nabla), \quad 0 \leq r \leq k+2$.

if $\left(M^{n}, g\right)$ satisfies $(I)$ and $\left(B_{k}\right)$.

Proposition 2.3. Let $(E, h, \nabla) \rightarrow\left(M^{n}, g\right)$ be a Riemannian vector bundle satisfying $(I),\left(B_{k}\left(M^{n}, g\right)\right)$ and $\left(B_{k}(E, \nabla)\right)$.

a. Assume $k \geq r, k \geq 1, r-\frac{n}{p} \geq s-\frac{n}{q}, r \geq s, q \geq p \geq 1$. Then the inclusion

$$
\Omega^{p, r}(E) \hookrightarrow \Omega^{q, s}(E)
$$

is continuous.

b. If $k \geq 0, r>\frac{n}{p}+s$, then the inclusion

$$
\Omega^{p, r}(E) \hookrightarrow^{b, s} \Omega(E)
$$

is continuous.

We refer to [12] for the proof.

A key role for everything below plays the module structure theorem for Sobolev spaces.

Theorem 2.4. Let $\left(E_{i}, h_{i}, \nabla_{i}\right) \rightarrow\left(M^{n}, g\right)$ be vector bundles with $(I)$, $\left(B_{k}\left(M^{n}, g\right)\right),\left(B_{k}\left(E_{i}, \nabla_{i}\right)\right), i=1,2$. Assume $0 \leq r \leq r_{1}, r_{2} \leq k$. If $r=0$ assume

$$
\begin{gathered}
\left\{\begin{aligned}
r-\frac{n}{p} & <r_{1}-\frac{n}{p_{1}} \\
r-\frac{n}{p} & <r_{2}-\frac{n}{p_{2}} \\
r-\frac{n}{p} & \leq r_{1}-\frac{n}{p_{1}}+r_{2}-\frac{n}{p_{2}} \\
\frac{1}{p} & \leq \frac{1}{p_{1}}+\frac{1}{p_{2}}
\end{aligned}\right\} \text { or } \\
\left\{\begin{aligned}
r-\frac{n}{p} \leq r_{1}-\frac{n}{p_{1}} \\
0<r_{2}-\frac{n}{p_{2}} \\
\frac{1}{p} \leq \frac{1}{p_{1}}
\end{aligned}\right\} \text { or }\left\{\begin{aligned}
0 & -\frac{n}{p_{1}} \\
r-\frac{n}{p} & \leq r_{2}-\frac{n}{p_{2}} \\
\frac{1}{p} & \leq \frac{1}{p_{2}}
\end{aligned}\right\} .
\end{gathered}
$$


If $r>0$ assume $\frac{1}{p} \leq \frac{1}{p_{1}}+\frac{1}{p_{2}}$ and

$$
\left\{\begin{aligned}
r-\frac{n}{p} & <r_{1}-\frac{n}{p_{1}} \\
r-\frac{n}{p} & <r_{2}-\frac{n}{p_{2}} \\
r-\frac{n}{p} & \leq r_{1}-\frac{n}{p_{1}}+r_{2}-\frac{n}{p_{2}}
\end{aligned}\right\} \text { or }\left\{\begin{aligned}
r-\frac{n}{p} \leq r_{1}-\frac{n}{p_{1}} \\
r-\frac{n}{p} \leq r_{2}-\frac{n}{p_{2}} \\
r-\frac{n}{p}<r_{1}-\frac{n}{p_{1}}+r_{2}-\frac{n}{p_{2}}
\end{aligned}\right\} \text {. }
$$

Then the tensor product of sections defines a continuous bilinear map

$$
\Omega^{p_{1}, r_{1}}\left(E_{1}, \nabla_{1}\right) \times \Omega^{p_{2}, r_{2}}\left(E_{2}, \nabla_{2}\right) \longrightarrow \Omega^{p, r}\left(E_{1} \otimes E_{2}, \nabla_{1} \otimes \nabla_{2}\right) .
$$

We refer to [12] for the proof.

Consider now $\left(M^{n}, g\right),\left(N^{n^{\prime}}, h\right)$ open, satisfying $(I),\left(B_{k}\right)$ and $f \in$ $C^{\infty}(M, N)$. Then the differential $d f=f_{*}$ is a section of $T^{*} M \otimes f^{*} T N$, where $f^{*} T N$ is endowed with the induced connection $f^{*} \nabla^{h}$. The connections $\nabla^{g}$ and $f^{*} \nabla^{h}$ induce connections $\nabla$ in all tensor bundles $T_{s}^{q}(M) \otimes f^{*} T_{v}^{u} N$. Therefore $\nabla^{m} d f$ is well defined. Assume $m \leq k$. We denote by $C^{\infty, m}(M, N)$ the set of all $f \in C^{\infty}(M, N)$ satisfying

$$
{ }^{b, m}|d f|:=\sum_{i=0}^{m-1} \sup _{x \in M}\left|\nabla^{i} d f\right|_{x}<\infty .
$$

Let $Y \in \Omega\left(f^{*} T N\right):=C^{\infty}\left(f^{*} T N\right)$. Then $Y_{x}$ can be written as $\left(Y_{f(x)}, x\right)$, and we define a map $f_{Y}: M \rightarrow N$ by

$$
f_{Y}(x):=(\exp Y)(x):=\exp Y_{x}:=\exp _{f(x)} Y_{f(x)} .
$$

Then the map $f_{Y}$ defines an element of $C^{\infty}(M, N)$. Moreover we have

Proposition 2.5. Assume $m \leq k$ and ${ }^{b, m}|Y|=\sum_{i=0}^{m} \sup _{x \in M}\left|\nabla^{i} Y\right|_{x}<\delta_{N}<$ $r_{i n j}(N), f \in C^{\infty, m}(M, N)$. Then

$$
f_{Y}=\exp Y \in C^{\infty, m}(M, N) .
$$

We refer to [10] for the proof. The main point is that one shows that $\left|\nabla^{u}(d \exp Y-d(i d))\right|$ makes sense and that

$$
\left|\nabla^{\mu}(d \exp Y-d(i d))\right| \leq P_{\mu}\left(\left|\nabla^{i} d f\right|,\left|\nabla^{j} Y\right|\right), \quad i \leq \mu, \quad j \leq \mu+1,
$$

where the $P_{\mu}$ are certain universal polynomials in the indicated variables without constant terms and each term has at least one $\left|\nabla^{j} Y\right|, 0 \leq j \leq \mu+1$ as a factor. 
Now we consider manifolds of maps in the $L_{p}$-category. Assume that $\left(M^{n}, g\right),\left(N^{n^{\prime}}, h\right)$ are open, of bounded geometry up to order $k, r \leq m \leq$ $k, 1 \leq p<\infty, r>\frac{n}{p}+1$. Consider $f \in C^{\infty, m}(M, N)$. According to 2.3, for $r>\frac{n}{p}+s$

$$
\begin{aligned}
& \Omega^{p, r}\left(f^{*} T N\right) \hookrightarrow{ }^{b, s} \Omega\left(f^{*} T N\right) \\
& { }^{b, s}|Y| \leq D \cdot|Y|_{p, r},
\end{aligned}
$$

where $|Y|_{p, r}=\left(\int \sum_{i=0}^{r}\left|\nabla^{i} Y\right|^{p} d v o l\right)^{\frac{1}{p}}$ and $\nabla=f^{*} \nabla^{h}$. Set for $\delta>0, \delta \cdot D \leq$ $\delta_{N}<r_{i n j}(N) / 2,1 \leq p<\infty$

$$
\begin{array}{r}
V_{\delta}:=\left\{(f, g) \in C^{\infty, m}(M, N)^{2} \mid \text { there exists } Y \in \Omega_{r}^{p}\left(f^{*} T N\right)\right. \text { such that } \\
\left.g=f_{Y}=\exp Y \text { and }|Y|_{p, r}<\delta\right\} .
\end{array}
$$

Theorem 2.6. Under the conditions above $\mathcal{V}:=\left\{V_{\delta}\right\}_{0<\delta<r_{\text {inj }}(N) / 2 D}$ is a basis for a metrizable uniform structure $\mathcal{A}^{p, r}\left(C^{\infty, m}(M, N)\right)$.

We refer to [12] for the rather complicated proof.

Let ${ }^{m} \Omega^{p, r}(M, N)$ be the completion of $C^{\infty, m}(M, N)$ with respect to this uniform structure. From now on we assume $r=m$ and denote $\Omega^{p, r}(M, N):=$ ${ }^{r} \Omega^{p, r}(M, N)$.

Theorem 2.7. Let $\left(M^{n}, g\right),\left(N^{n^{\prime}}, h\right)$ be open and of bounded geometry of order $k, 1 \leq p<\infty, k \geq r>\frac{n}{p}+1$. Then each component of $\Omega^{p, r}(M, N)$ is a $C^{k+1-r}-$ Banach manifold, and for $p=2$ it is a Hilbert manifold.

We refer to [12] for the proof.

Let $\left(M^{n}, g\right)$ be as above. A choice of an orthonormal basis in each $T_{x} M$ implies that $|\lambda|_{\min }(d f)$, the minimum of the absolute value of the eigenvalues of the Jacobian of $f$, is well defined. Set

$$
\mathcal{D}^{p, r}:=\left\{f \in \Omega^{p, r}(M, N) \mid f \text { is injective, surjective and }|\lambda|_{\min }(d f)>0\right\} .
$$

Theorem 2.8. $\mathcal{D}^{p, r}$ is open in $\Omega^{p, r}(M, N)$. In particular, each component is a $C^{k+1-r}-$ Banach manifold, and for $p=2$ it is a Hilbert manifold. 
Theorem 2.9. Assume $\left(M^{n}, g\right), k, p, r$ as above.

a. Assume $f, h \in \mathcal{D}^{p, r}, h \in \operatorname{comp}\left(i d_{M}\right) \subset \mathcal{D}^{p, r}$. Then $h \circ f \in \mathcal{D}^{p, r}$ and $h \circ f \in \operatorname{comp}(f)$.

b. Assume $f \in \operatorname{comp}\left(i d_{M}\right) \subset \mathcal{D}^{p, r}$. Then $f^{-1} \in \operatorname{comp}\left(i d_{M}\right) \subset \mathcal{D}^{p, r}$.

c. $\mathcal{D}_{0}^{p, r}:=\operatorname{comp}\left(i d_{M}\right)$ is a metrizable topological group.

Theorem 2.10 ( $\alpha$-lemma). Assume $k \geq r>\frac{n}{p}+1, f \in \mathcal{D}^{p, r}$. Then the right multiplication $\alpha_{f}: \mathcal{D}_{0}^{p, r} \rightarrow \mathcal{D}^{p, r}, \alpha_{f}(h)=h \circ f$, is of class $C^{k+1-r}$.

Theorem 2.11 ( $\omega$-lemma). Let $k+1-(r+s)>s, f \in \mathcal{D}_{0}^{p, r+s} \subset \mathcal{D}_{0}^{p, r}$, $r>\frac{n}{p}+1$. Then the left multiplication $\omega_{f}: \mathcal{D}^{p, r} \rightarrow \mathcal{D}^{p, r}, \omega_{f}(h)=f \circ h$, is of class $C^{s}$.

We defined for $C^{\infty, m}(M, N)$ a uniform structure $\mathcal{A}^{p, r}$. Consider now $C^{\infty, \infty}(M, N)=\bigcap_{m} C^{\infty, m}(M, N)$. Then we have an inclusion $i$ : $C^{\infty, \infty}(M, N) \hookrightarrow C^{\infty, m}(M, N)$ and hence a well defined uniform structure $\mathcal{A}^{\infty, p, r}=(i \times i)^{-1} \mathcal{A}^{p, r}$. After completion we obtain once again the manifold $\Omega^{\infty, p, r}(M, N)$, where $f \in \Omega^{\infty, p, r}(M, N)$ if and only if for every $\varepsilon>0$ there exist an $\tilde{f} \in C^{\infty, \infty}(M, N)$ and a $Y \in \Omega^{p, r}\left(\tilde{f}^{*} T N\right)$ such that $f=\exp Y$ and $|Y|_{p, r} \leq \varepsilon$. Moreover, each component of $\Omega^{\infty, p, r}(M, N)$ is a Banach manifold and $T_{f} \Omega^{\infty, p, r}(M, N)=\Omega^{p, r}\left(f^{*} T N\right)$. As above we set

$$
\begin{aligned}
\mathcal{D}^{\infty, p, r}=\left\{f \in \Omega^{\infty, p, r}(M, N) \mid f\right. \text { is injective, surjective } \\
\left.\quad \text { and }|\lambda|_{\min }(d f)>0\right\} .
\end{aligned}
$$

Theorem 2.12. Assume the conditions for defining $\mathcal{D}^{p, r}$. Then

$$
\mathcal{D}_{0}^{\infty, p, r}=\mathcal{D}_{0}^{p, r}
$$

We refer to [15], p. 163 for the proof.

\section{The Group of Contact Transformations.}

From now on we restrict ourselves to $p=2$ and write $\mathcal{D}_{0}^{r} \equiv \mathcal{D}_{0}^{2, r}$. Moreover, we have to consider $q$-forms with values in a vector bundle $E$, i. e. elements of $\Omega^{q, 2, r}(E) \equiv \Omega^{2, r}\left(\Lambda^{q} T^{*} M \otimes E\right)$. Sections of $E$ are simply 0 -forms with values in $E$. Usual forms on $M$ are forms with values in $M \times R \rightarrow M$ and we write simply $\Omega^{q, 2, r} \equiv \Omega^{q, 2, r}(M)$. 
In [15] we studied the group $\mathcal{D}_{\omega, 0}^{r+1}$ of form preserving diffeomorphisms $f \in \mathcal{D}_{0}^{r+1}, f^{*} \omega=\omega, \omega$ a symplectic or volume form. We proved the following

Theorem 3.1. Assume $\left(M^{n}, g\right)$ with $(I),\left(B_{\infty}\right), \omega \in \in^{b, m} \Omega^{q}$ for all $m$, closed and strongly nondegenerate, $q=n$ or $q=2$, $\inf \sigma_{e}\left(\left.\triangle_{1}\right|_{\left(\operatorname{ker} \triangle_{1}\right)^{\perp}}\right)>0$. Let $\mathcal{D}_{\omega, 0}^{\infty}=\lim _{\leftarrow} \mathcal{D}_{\omega, 0}^{r}$. Then $\left\{\mathcal{D}_{\omega, 0}^{\infty}, \mathcal{D}_{\omega, 0}^{r} \mid r>\frac{n}{2}+1\right\}$ is an ILH Lie group in the sense of [17], [3] and the Lie algebra of $\mathcal{D}_{\omega, 0}^{\infty}$ consists of divergence free $(q=n)$ or locally Hamiltonian $(q=2)$ vector fields $X$, respectively, with $|X|_{2, r}<\infty$ for all $r$.

Here strongly nondegenerate means that $\inf _{x \in M}|\omega|_{x}>0$.

A similar theorem for the group of contact transformations would be desirable and will be necessary for the constructions in Sections 5 and 6 . As a result of our efforts, such a theorem can be established but it is once again a long, complicated story and will appear together with results on other diffeomorphism groups in [13]. Hence we only sketch the proof here.

Let $\left(M^{2 n+1}, g_{0}, \theta\right)$ be an oriented Riemannian contact manifold, $g_{0}$ satisfying $(I)$ and $\left(B_{k+2}\right)$ and $\theta$ a contact form. We assume additionally ${ }^{b, k+3}|\theta|_{g_{0}}<\infty$ and $\inf _{x}|d \theta|_{x}>0 . \quad \theta$ is a 1 -form with $\mu:=\theta \wedge(d \theta)^{n} \neq 0$ everywhere and we assume that $\mu$ coincides with the given orientation. $\theta$ defines the Reeb vector field $\xi$ on $M, \theta(\xi)=1, i_{\xi} d \theta=0$. Denote $E=\operatorname{ker} \theta$. Clearly $T M=R \xi \oplus E$. A Riemannian metric $g$ is called associated to $\theta$ if there exists a $(1,1)$ tensor field $\varphi$ on $M$ such that for any vector fields $X, Y$ on $M$ we have

1) $g(X, \xi)=\theta(X)$

2) $\varphi^{2}=-I+\theta \otimes \xi$

3) $d \theta(X, Y)=g(X, \varphi Y)$.

These conditions imply

4) $g(\xi, \xi)=1$,

5) $E \perp \xi$,

6) $\varphi(\xi)=0, \varphi(E)=E$,

7) $d \theta(\varphi X, \varphi Y)=d \theta(X, Y)$

8) $g(X, Y)=\theta(X) \cdot \theta(Y)+d \theta(\varphi X, Y)$.

Given $g_{0}$ with $(I)$ and $\left(B_{k+2}\right), \theta$ as above, we want to construct a metric $g$ of bounded geometry associated to $\theta$. This can be achieved as follows:

Proposition 3.1. Assume $\left(M^{2 n+1}, g_{0}, \theta\right)$ as above. Then there exists a metric $g$ associated to $\theta$ satisfying $(I),\left(B_{k}\right)$ and ${ }^{b, k}|\theta|_{g}<\infty$. 
Proof. We sketch the simple proof. Start with $g_{0}$ and define $h$ by $h(X, Y)=$ $g_{0}(-X+\theta(X) \xi,-Y+\theta(Y) \xi)+\theta(X) \theta(Y)$. Then $\xi \perp_{h} \operatorname{ker} \theta$ and $|\xi|_{h}=1$. Let $\left(X_{1}, \ldots, X_{2 n}, \xi\right)$ be a local orthonormal basis with respect to $h$ and write

$$
\left((d \theta)_{i j}\right)=\left(d \theta\left(X_{i}, Y_{j}\right)\right)=F \cdot G,
$$

where $F$ is orthogonal and $G$ is symmetric and positive definite. Then, according to [6], $\left(\begin{array}{cc}G & 0 \\ 0 & 1\end{array}\right)$ defines a Riemannian metric $g$ on $M$ and $\left(\begin{array}{cc}F & 0 \\ 0 & 0\end{array}\right)$ defines a global $(1,1)$ tensor field $\varphi$ with $\varphi^{2}=-I+\theta \otimes \xi$ and $d \theta(X, Y)=g(X, \varphi Y)$.

Denote by " $"$ " in a symbolic notation the (euclidean) differentiation. Then $(G)^{\prime}=\left(\frac{(d \theta)_{i j}}{F}\right)^{\prime}=\left((d \theta)_{i j}\right)^{\prime} \cdot \frac{1}{F}-\left((d \theta)_{i j}\right) \cdot \frac{F^{\prime}}{F^{2}}$. Similarly for higher derivatives. This implies $\left(B_{k}\right)$ for $g$. Finally the condition $(I)$ for $g_{0}$ and the fact that replacement of $g_{0}$ by $g$ implies uniformly boundedness from above and below and the change of local volumes yields (I) for $g$. Here we use theorem 4.7 of [7].

From now on we assume $g$ with conditions $(I),\left(B_{k}\right)$ and properties 1)-8), $k \geq r+1,{ }^{b, r+1}|\theta|_{g}<\infty, r+1>\frac{2 n+1}{2}+2$. Consider the space

$$
\mathcal{F}:=\left\{\alpha \in C^{\infty}(M)\left|{ }^{b, r+1}\right| \alpha \mid<\infty\right\} .
$$

Set for $\delta>0$

$$
\begin{aligned}
V_{\delta}:=\left\{\left(\alpha_{1}, \alpha_{2}\right) \in \mathcal{F}^{2} \mid\right. & \left|\alpha_{1}-\alpha_{2}\right|_{2, r+1} \\
& \left.=\left(\int \sum_{i=0}^{r+1}\left|\left(\nabla^{g}\right)^{i}\left(\alpha_{1}-\alpha_{2}\right)\right|_{g, x}^{2} d v o l_{x}(g)\right)^{\frac{1}{p}}<\delta\right\} .
\end{aligned}
$$

Lemma 3.2. $\mathcal{B}=\left\{V_{\delta}\right\}_{\delta>0}$ is a basis for a metrizable uniform structure on the space $\mathcal{F}$.

Let $\mathcal{F}^{r+1}$ be the completion of $\mathcal{F}$ with respect to $\mathcal{B}$. Then $\mathcal{F}^{r+1}$ is locally contractible, hence locally arcwise connected, hence components coincide with arc components. The elements of $\mathcal{F}$ are dense in each component. 
Proposition 3.3. Let $\alpha \in \mathcal{F}$. Then the component of $\alpha$ is given by

$$
\operatorname{comp}(\alpha)=\left\{\alpha^{\prime} \in \mathcal{F}^{r+1}|| \alpha-\left.\alpha^{\prime}\right|_{2, r+1}<\infty\right\}=\alpha+\Omega^{0,2, r+1}(M) .
$$

In particular each component is open and a Hilbert manifold modeled over $\Omega^{0,2, r+1}(M)$.

Remark. For $\alpha=0$ the component $\operatorname{comp}(\alpha)$ coincides with the Sobolev space $\Omega^{0,2, r+1}(M)$, but for nonzero $\alpha$ its component comp $(\alpha)$ equals to the affine space $\alpha+\Omega^{0,2, r+1}(M)$.

Corollary 3.4. $\mathcal{F}^{r+1}$ has a representation as a topological sum of its components,

$$
\mathcal{F}^{r+1}=\sum_{i \in I} \operatorname{comp}\left(\alpha_{i}\right)
$$

Set $\mathcal{F}_{0}^{r+1}=\left\{\alpha \in \operatorname{comp}(1) \mid \inf _{x \in M} \alpha(x)>0\right\}$. Then $\mathcal{F}_{0}^{r+1}$ is an open subset of comp (1), in particular $T_{\alpha} \mathcal{F}_{0}^{r+1}=\Omega^{0,2, r+1}(M), \alpha \in \mathcal{F}_{0}^{r+1}$. Moreover, $\mathcal{F}_{0}^{r+1}$ is a Hilbert Lie group.

Now we define

$$
\mathcal{D}_{\theta, 0}^{r+1}:=\left\{(\alpha, f) \in \mathcal{F}_{0}^{r+1} \rtimes \mathcal{D}_{0}^{r+1} \mid \alpha f^{*} \theta=\theta\right\},
$$

where $>\triangleleft$ denotes the semidirect product.

Proposition 3.5. $\mathcal{D}_{\theta, 0}^{r+1}$ is a closed subgroup of $\mathcal{F}_{0}^{r+1} \rtimes \mathcal{D}_{0}^{r+1}$ and a topological group.

Theorem 3.6. Assume $\left(M^{2 n+1}, g, \theta\right)$ with $(I),\left(B_{k}\right), g$ associated to $\theta, k \geq$ $r+1>\frac{2 n+1}{2}+2,{ }^{b, r+1}|\theta|<\infty$ and $\left.\inf \sigma_{e}\left(\triangle_{1}\right)\right|_{\left(\operatorname{ker} \triangle_{1}\right)^{\perp}}>0$. Then $\mathcal{D}_{\theta, 0}^{r+1}$ is a closed $C^{k-r}$ Hilbert submanifold of $\mathcal{F}_{0}^{r+1} \rtimes \mathcal{D}_{0}^{r+1}$.

The sketched proof will occupy the remaining part of this section. As usual, we will show that $\mathcal{D}_{\theta, 0}^{r+1}$ is the preimage of a point by a submersion.

Lemma 3.7. Let $\alpha \in \mathcal{F}_{0}^{r+1}, f \in \mathcal{D}_{0}^{r+1}$. Then

$$
\alpha f^{*} \theta-\theta \in \Omega^{1,2, r}
$$

and

$$
d \alpha \wedge f^{*} \theta-\alpha f^{*} d \theta-d \theta \in \Omega^{2,2, r}
$$


Proof. The proof will be based on the Lemmas 3.8-3.10.

Write

$$
\begin{aligned}
\alpha f^{*} \theta-\theta & =\alpha\left(f^{*} \theta-\theta\right)+(\alpha-1) \theta \\
\alpha\left(f^{*} \theta-\theta\right) & =(\alpha-1)\left(f^{*} \theta-\theta\right)+f^{*} \theta-\theta .
\end{aligned}
$$

Assume $f^{*} \theta-\theta \in \Omega^{1,2, r}$. We have $(\alpha-1) \in \Omega^{0,2, r+1}$. The module structure theorem 2.4 applied to $(\alpha-1)\left(f^{*} \theta-\theta\right)$ gives $(\alpha-1)\left(f^{*} \theta-\theta\right) \in \Omega^{1,2, r}$ and $\alpha\left(f^{*} \theta-\theta\right) \in \Omega^{1,2, r}$. Moreover, $(\alpha-1) \theta \in \Omega^{1,2, r}$ since $(\alpha-1) \in \Omega^{0,2, r+1}$ and ${ }^{b, r+1}|\theta|<\infty$.

Hence the proof of (3.1) reduces to the following

Lemma 3.8. Assume $f \in \mathcal{D}_{0}^{r+1}$. Then $f^{*} \theta-\theta \in \Omega^{1,2, r}$.

Proof. Any $f \in \mathcal{D}_{0}^{r+1}$ has a representation $f=\exp X_{u} \circ \cdots \circ \exp X_{1}$. We start with the simplest case $f=\exp X, X \in \Omega^{0,2, r+1}(T M)$. The main steps in the proof are done already in [15]. We recall them. Let $I=[0,1]$ and $i_{t}: M \rightarrow I \times M$ the embedding $i_{t}(x)=(t, x)$. We put on $I \times M$ the product metric $\left(\begin{array}{ll}1 & 0 \\ 0 & g\end{array}\right)$.

Lemma 3.9. For every $q \geq 0$ there exists a linear bounded mapping

$$
K:{ }^{b, m} \Omega^{q+1}(I \times M) \rightarrow{ }^{b, m} \Omega^{q}(M)
$$

such that $d K+K d=i_{1}^{*}-i_{0}^{*}$.

This is Lemma 3.1 of [15].

Lemma 3.10. Let $f, h: M \rightarrow N$ be $C^{1}$-mappings and $F: I \times M \rightarrow N a$ $C^{1}$-homotopy between $f$ and $h$. Let

$$
f^{*}, h^{*}:{ }^{b, 1} \Omega^{q}(N) \rightarrow{ }^{b, 1} \Omega^{q}(M), \quad F^{*}:{ }^{b, 1} \Omega^{q}(N) \rightarrow{ }^{b, 1} \Omega^{q}(I \times M)
$$

be bounded. Then for $\Phi \in{ }^{b, 1} \Omega^{q}(N)$

$$
\left(h^{*}-f^{*}\right) \Phi=(d K+K d) F^{*} \Phi .
$$

This is Lemma 3.2 of [15]. 
Hence we have to estimate $(d K+K d) F^{*} \theta$ in our case $h=i d, f=\exp X$, $F=\exp t X$. This is done in Theorem 3.2 of [15] and its proof, [15] pp. 154158. The proof is rather involved. We conclude

$$
(\exp X)^{*} \theta-\theta \in \Omega^{1,2, r} .
$$

Assume now $f=\exp X_{n} \circ \cdots \circ \exp X_{1}$. A simple induction now yields $f^{*} \theta-\theta \in \Omega^{1,2, r}$ (cf. [15] p. 160). This finishes the proof of Lemma 3.8 and hence of (3.1).

Now we consider (3.2) which is the differential of (3.1). From this it is clear that the expression (3.2) is in $\Omega^{2,2, r-1}$. But can we prove more.

$$
d \alpha \wedge f^{*} \theta+\alpha f^{*} d \theta-d \theta=d(\alpha-1) \wedge f^{*} \theta+(\alpha-1) f^{*} d \theta+f^{*} d \theta-d \theta
$$

and

$$
\begin{gathered}
d(\alpha-1) \wedge f^{*} \theta=d(\alpha-1) \wedge\left(f^{*} \theta-\theta\right)+d(\alpha-1) \wedge \theta, \\
(\alpha-1) f^{*} d \theta=(\alpha-1)\left(f^{*} d \theta-d \theta\right)+(\alpha-1) d \theta .
\end{gathered}
$$

Now we use that $(\alpha-1) \in \Omega^{0,2, r}$ and $f^{*} d \theta \in \Omega^{2,2, r}$ (according to the first part of the proof) and $d \theta \in{ }^{b, r} \Omega^{2}$. Application of the module structure Theorem 2.4 yields the assertion. This finishes the proof of Lemma 3.7.

Define

$$
\begin{gathered}
\Psi: \mathcal{F}_{0}^{r+1} \rtimes \mathcal{D}_{0}^{r+1} \rightarrow \Omega^{1,2, r} \oplus \Omega^{2,2, r}, \\
\Psi(\alpha, f):=\left(\alpha f^{*} \theta-\theta, d \alpha \wedge f^{*} \theta+\alpha f^{*} \theta-d \theta\right) .
\end{gathered}
$$

Lemma 3.11. The map $\Psi$ is of class $k-r$.

We omit the considerations and estimates, refer to [13] and discuss in the sequel only the special case of $\left.D \Psi\right|_{(1, i d)}$.

Lemma 3.12. Let $A: \Omega^{0,2, r+1} \oplus \Omega^{0,2, r+1}(T M) \rightarrow \Omega^{1,2, r} \oplus \Omega^{2,2, r}$ be defined by

$$
A(u, X):=\left(u \cdot \theta+L_{X} \theta, d(u \cdot \theta)+d\left(i_{X} d \theta\right)\right) .
$$

Then

$$
\left.D \Psi\right|_{(1, i d)}(u, X)=A(u, X)
$$


Proof. From the facts that ${ }^{b, r+1}|\theta|<\infty, u \in \Omega^{0,2, r+1}$ and $X \in \Omega^{0,2, r+1}(T M)$ follows immediately that $u \cdot \theta, L_{X} \theta \in \Omega^{1,2, r}$ and $d(u \cdot \theta), d\left(i_{X} d \theta\right) \in \Omega^{2,2, r}$. Considering $\left.\frac{d}{d t} \Psi(1+t \cdot u, i d)\right|_{t=0}$, and $\left.\frac{d}{d \tau} \Psi(1, \exp \tau X)\right|_{\tau=0}$ yields the desired result.

Define

$$
B: \Omega^{1,2, r} \oplus \Omega^{2,2, r} \longrightarrow \Omega^{2,2, r-1} \oplus \Omega^{3,2, r-1}
$$

by

$$
B(\rho, \sigma):=(d \rho-\sigma, d \sigma)
$$

Clearly $B A=0$.

Lemma 3.13. Let the adjoints $A^{*}, B^{*}$ be defined with respect to the $L_{2}$ scalar product of forms. Then for $\square:=A A^{*}+B B^{*}$, we have

$$
\square(\rho, \sigma)=(\triangle \rho+\rho, \triangle \sigma+\sigma) .
$$

We refer to Lemma 8.3.2 of [17].

Corollary 3.14. The operator $\square$ is extendable to any Sobolev space of order $\leq k$ and

$$
\square: \Omega^{1,2, r} \oplus \Omega^{2,2, r} \longrightarrow \Omega^{2,2, r-2} \oplus \Omega^{3,2, r-2}
$$

is injective, surjective and bounded.

Consider ker $B \subset \Omega^{1,2, r} \oplus \Omega^{2,2, r}$. Then $\Psi$ maps $\mathcal{F}^{r+1} \rtimes \mathcal{D}_{0}^{r+1}$ into $\Omega^{1,2, r} \oplus$ $\Omega^{2,2, r}$. The following is immediately clear from the definitions.

Lemma 3.15. $i m \Psi \subseteq \operatorname{ker} B$

Proposition 3.16. Assume that $\inf \sigma_{e}\left(\left.\triangle_{1}\right|_{\left(\operatorname{ker} \triangle_{1}\right)^{\perp}}\right)>0$. Then the operator

$$
\left.D \Psi\right|_{(1, i d)}=A: \Omega^{0,2, r+1} \oplus \Omega^{0,2, r+1}(T M) \longrightarrow T_{(0,0)} \operatorname{ker} B=\operatorname{ker} B
$$

is surjective.

Proof. Consider

$$
\Omega^{0,2, r+1} \oplus \Omega^{2,2, r+1}(T M) \stackrel{A_{l}}{\longrightarrow} \Omega^{1,2, r} \oplus \Omega^{2,2, r} \stackrel{B}{\longrightarrow} \Omega^{2,2, r-1} \oplus \Omega^{3,2, r-1} .
$$


This is an elliptic complex. Hence

$$
\Omega^{1,2, r} \oplus \Omega^{2,2, r}=\operatorname{ker} \square \oplus \overline{i m A} \oplus \overline{i m B^{*}}=\overline{i m A} \oplus \overline{i n B^{*}},
$$

where the summands are $L_{2}$-orthogonal and the completion is taken with respect to ||$_{2, r}$. Moreover, it is easy to see that $\operatorname{ker} B \subseteq \overline{i m A}$. Hence we are done if we can show that

$$
\overline{i m A}=i m A \text {. }
$$

Now it is a well known fact from elementary functional analysis that $A$ is closed if and only if $i m A A^{*}$ is closed. A longer calculation yields

$$
A A^{*}(\rho, \sigma)=(d \delta \rho+\rho+\delta \sigma, d \delta \sigma+d \rho) .
$$

Hence $i m A A^{*}$ is closed if and only if the operators

$$
(\rho, \sigma) \longrightarrow d \delta \rho+\rho+\delta \sigma
$$

and

$$
(\rho, \sigma) \longrightarrow d \delta \sigma+d \rho
$$

have closed image, respectively. Now a careful analysis shows that this is the case if $i m \triangle_{1}$ is closed. The latter is equivalent to inf $\sigma_{e}\left(\left.\triangle_{1}\right|_{\left(\operatorname{ker} \triangle_{1}\right)^{\perp}}\right)>0$. We refer to [13] for details. This finishes the proof of proposition 3.16.

A series of shifting arguments yield the same result at any other point $(\alpha, f)$, i.e., $\left.D \Psi\right|_{(\alpha, f)}$ is surjective. Hence $\Psi$ is a submersion and

$$
\mathcal{D}_{\theta, 0}^{r+1}=\Psi^{-1}(0,0)
$$

is closed submanifold. The proof of Proposition 3.6 is finished.

Corollary 3.17. Assume $\left(M^{2 n+1}, g, \theta\right)$ satisfying $(I),\left(B_{\infty}\right), \sup _{x \in M}\left|\nabla^{i} \theta\right|_{x}<$ $\infty$ for all $i$ and $\inf \sigma_{e}\left(\left.\triangle_{1}\right|_{\left(\operatorname{ker} \triangle_{1}\right)^{\perp}}\right)>0$. Set $\mathcal{D}_{\theta, 0}^{\infty}:=\lim _{\overleftarrow{r}} \mathcal{D}_{\theta, 0}^{r+1}$. Then

$$
\left\{\mathcal{D}_{\theta, 0}^{\infty}, \mathcal{D}_{\theta, 0}^{r+1} \mid r+1 \geq \frac{2 n+1}{2}+2\right\}
$$

is an ILH Lie group. 


\section{Contact Transformations of the Restricted Cotangent Bundle $T^{*} M \backslash 0$.}

The most important example for us of contact manifolds of bounded geometry is the cotangent sphere bundle $S=S\left(T^{*} M\right) \cong\left(\left(T^{*} M\right) \backslash 0\right) / \mathbf{R}_{+}$. We consider the Sasaki metric on $T^{*} M$. Let $\pi: T^{*} M \rightarrow M$ be the projection and $K$ the connection map of the Levi-Civita connection in the cotangent bundle. Then the Sasaki metric is defined by

$$
g_{T^{*} M}(X, Y)=g_{M}\left(\pi_{*} X, \pi_{*} Y\right)+g_{M}(K X, K Y), \quad X, Y \in T T^{*} M
$$

Let $g_{S}:=\left.g_{T^{*} M}\right|_{S\left(T^{*} M\right)}$ be the restriction of the Sasaki metric to the cosphere bundle.

Lemma 4.1. If $\left(M, g_{M}\right)$ satisfies $(I),\left(B_{k+1}\right), 0 \leq k \leq \infty$ fixed, then $\left(S\left(T^{*} M\right), g_{S}\right)$ satisfies $(I),\left(B_{k}\right)$.

We refer to [15], p. 165 for the proof.

Let $\theta$ be the canonical one form on $T^{*} M$, i. e. for $X \in T_{z} T^{*} M, \theta(X):=$ $z\left(\pi_{*} X\right)$. Then $\theta_{s}=i^{*} \theta$ is a contact form on $S\left(T^{*} M\right)$, where $i: S\left(T^{*} M\right) \rightarrow$ $T^{*} M$ is the inclusion. As pointed out in [5], $g_{S}$ and $\theta_{S}$ are not associated but this is true for $g_{S}^{\prime}:=\frac{1}{4} g_{S}$ and $\theta_{S}^{\prime}:=\frac{1}{2} \theta_{S}$. In [5], pp. 132-135 the Reeb vector field $\xi$, the $(1,1)$ tensor field $\varphi$ and the covariant derivatives are explicitly calculated. Denote for the sake of simplicity the new $\theta_{S}^{\prime}$ from now on by $\theta=\theta_{S}^{\prime}$ and $g_{S}^{\prime}$ by $g_{S}$.

Lemma 4.2. Suppose $(M, g)$ with $(I),\left(B_{k+2}\right)$. Then $\theta=\theta_{s} \in{ }^{b, k+1} \Omega^{1}$, i.e., $\sup _{z \in S}\left|\nabla^{i} \theta\right|_{g_{S}, z}<\infty, 0 \leq i \leq k+1$.

Proof. Start with $i=0$. Let $e_{1}, \ldots, e_{2 n-1}$ be an orthonormal basis in $T_{z_{0}} S$ such that $e_{2 n-1}=\xi$. Then

$$
|\Omega|_{g_{S}, z_{0}}^{2}=\sum_{i=1}^{2 n-1} \Omega_{z_{0}}\left(e_{i}\right)^{2}=1
$$

It is well known that on a contact Riemannian manifold the integral curves of the Reeb vector field $\xi$ are geodesics (cf. [5] p. 54), i.e., $\nabla_{\xi} \xi=0$. Fix 
at $z_{0}$ the orthonormal basis $e_{1}, \ldots, e_{2 n-1}, e_{2 n-1}=\xi$. According to $\theta(X)=$ $g(\xi, X), \theta$ is the covariant form of $\xi$. Hence $\left|\nabla^{\nu} \theta\right|=\left|\nabla^{\nu} \xi\right|$.

$$
|\nabla \theta|^{2}=|\nabla \xi|^{2}=\sum_{i=1}^{2 n-1}\left|\nabla_{e_{i}} \cdot \xi\right|^{2}=\sum_{i=1}^{2 n-2}\left|\nabla_{e_{i}} \cdot \xi\right|^{2} .
$$

According to [5], p. 133-135, formulas (3)-(8),

$$
\begin{aligned}
\left|\nabla_{e_{i}} \cdot \xi\right| & \leq C_{1}^{\prime} \cdot|\varphi|+C_{2}^{\prime}\left|R^{g_{S}}\right|, \quad|\nabla \xi| \leq C_{1}|\varphi|+C_{2}\left|R^{g_{S}}\right| \\
\left|\nabla^{\nu} \theta\right| & \leq C_{1, \nu}\left|\nabla^{\nu-1} \varphi\right|+C_{2, \nu}\left|\nabla^{\nu-1} R^{g_{S}}\right| \\
\left|\nabla^{\mu} \varphi\right| & \leq D_{1, \mu}\left|\nabla^{\mu-1} \varphi\right|+D_{2, \mu}\left|\nabla^{\mu-1} R^{g_{S}}\right|
\end{aligned}
$$

which yields together with 4.1 the assertion.

Theorem 4.3. Suppose $\left(M^{n}, g\right)$ with $(I)$ and $\left(B_{k+1}\right), k \geq r+1>\frac{2 n-1}{2}+$ 2 , and $\left.\inf \sigma_{e}\left(\triangle_{1}\left(g_{S}\right)\right)\right|_{\left.\left(\operatorname{ker} \triangle_{1}\left(g_{S}\right)\right)^{\perp}\right)}>0$. Then $\mathcal{D}_{\theta, 0}^{r+1}\left(S\left(T^{*} M\right), g_{S}\right)$ is well defined and a $C^{k-r}$ submanifold of $\mathcal{F}_{0}^{r+1} \rtimes \mathcal{D}_{0}^{r+1}$.

This follows immediately from Theorem 3.6.

Corollary 4.4. Suppose $\left(M^{n}, g\right)$ with $(I)$ and $\left(B_{\infty}\right)$ and

$$
\left.\inf \sigma_{e}\left(\triangle_{1}\left(g_{S}\right)\right)\right|_{\left.\left(\operatorname{ker} \triangle_{1}\left(g_{S}\right)\right)^{\perp}\right)}>0 .
$$

Set $\mathcal{D}_{\theta, 0}^{\infty}:=\lim _{\leftarrow} \mathcal{D}_{\theta, 0}^{r+1}$. Then $\left\{\mathcal{D}_{\theta, 0}^{\infty}, \mathcal{D}_{\theta, 0}^{r+1} \mid r+1>\frac{2 n-1}{2}+2\right\}$ is an ILH Lie group.

For our later applications we must rewrite 4.3 and 4.4 by rewriting $\mathcal{D}_{\theta, 0}^{r+1}\left(S\left(T^{*} M\right), g_{S}\right)$ in an isomorphic version as (writing $\mathcal{D}_{\theta, 0}^{r+1}(S)$ for short)

$$
\mathcal{D}_{\theta, 0}^{r+1}(S)=\left\{(f, \beta) \in \mathcal{D}_{0}^{r+1}(S) \rtimes \mathcal{F}_{0}^{r+1}(S) \mid f^{*} \theta=\beta \theta\right\},
$$

where $(\alpha, f) \mapsto\left(f, \alpha^{-1}\right)$ is the canonical isomorphism (w.r.t. $\alpha$ antiisomorphism). The "Lie algebra" of $\mathcal{D}_{\theta, 0}^{r+1}\left(S\left(T^{*} M\right)\right)$ is

$$
\mathbf{d}_{\theta, 0}^{r+1}(S)=\left\{(X, u) \in \Omega^{0,2, r+1}(T S) \rtimes \Omega^{0,2, r+1}(S) \mid L_{X} \theta=u \cdot \theta\right\}
$$

with

$$
[(X, u),(Y, v)]=([X, Y], X(v)-Y(u)) .
$$


From the last equation it is clear that it isn't a Lie algebra since the bracket decreases the Sobolev index. It is only the tangent space at $(i d, 1)$.

Define now a map $\Phi$ from $\mathcal{D}_{\theta, 0}^{r+1}(S)$ into the homogeneous of degree one $C^{2}$ diffeomorphisms $\tilde{f}$ of $T^{*} \backslash 0$ satisfying $\tilde{f}^{*} \theta=\theta$ (cf. 4.7 below). Given $(f, \beta) \in \mathcal{D}_{\theta, 0}^{r+1}(S)$, we define $\tilde{f}=\Phi(f, \beta)$ by

$$
\tilde{f}(z):=\frac{f\left(\frac{z}{|z|}\right) \cdot|z|}{\beta\left(\frac{z}{|z|}\right)}, \quad z \in T^{*} M \backslash 0 .
$$

From $\tilde{f} \in i m \Phi$ we can reproduce $(f, \beta)=\Phi^{-1}(\tilde{f})$ by

$$
\begin{aligned}
& f\left(\frac{z}{|z|}\right)=\frac{\tilde{f}(z)}{|\tilde{f}(z)|}=\frac{\tilde{f}\left(\frac{z}{|z|}\right)}{\left|\tilde{f}\left(\frac{z}{|z|}\right)\right|}=\frac{\left.\tilde{f}\right|_{S}\left(\frac{z}{|z|}\right)}{|\tilde{f}|_{S}\left(\frac{z}{|z|}\right) \mid}, \\
& \beta\left(\frac{z}{|z|}\right)=\frac{|z|}{|\tilde{f}(z)|}=\frac{\left|\frac{z}{|z|}\right|}{\left|\tilde{f}\left(\frac{z}{|z|}\right)\right|}=\frac{1}{|\tilde{f}|_{S}\left(\frac{z}{|z|}\right) \mid} .
\end{aligned}
$$

For our applications below we recall two lemmas from [18].

Lemma 4.5. Let $f: T^{*} M \backslash 0 \rightarrow T^{*} M \backslash 0$ be a diffeomorphism. Then the following conditions are equivalent

a) $f^{*} \theta=\theta$

b) $f$ is symplectic (i.e., $f^{*} \omega=\omega, \omega=-d \theta$ ) and homogeneous of degree one.

\section{Lemma 4.6.}

a) Let $H: T^{*} M \backslash 0 \rightarrow R$ be homogeneous of degree $\nu$. Then the Hamiltonian vector field $X_{H}$ is homogeneous of degree $\nu-1$ and $\theta\left(X_{H}\right)=H$.

b) A vector field $X$ on $T^{*} M \backslash 0$ is homogeneous of degree zero if and only if its flow is homogeneous of degree one.

c) $L_{X} \theta=0$ if and only if $X$ is globally Hamiltonian, homogeneous of degree zero with Hamiltonian $\theta(X)$ homogeneous of degree one.

Proof. For later use we recall the proof of c). Let $L_{X} \theta=0, F_{t}$ the flow of $X$. Then $F_{t}^{*} \theta=\theta$. Lemma 4.5 implies that $F_{t}$ is symplectic and homogeneous 
of degree one and hence, according to b), $X$ is of degree zero. $0=L_{X} \theta=$ $i_{X} d \theta+d i_{X} \theta$ yields $i_{X} \omega=d \theta(X)$, i.e., $X=X_{H}$ with $H=\theta(X)$ homogeneous of degree one. The converse implication follows from a).

Define now

$$
\begin{aligned}
\mathcal{D}_{\theta, 0}^{r+1}\left(T^{*} M \backslash 0\right) & \\
& :=\left\{\tilde{f}: T^{*} M \backslash 0 \cong T^{*} M \backslash 0 \mid \tilde{f}=\Phi(f, \beta), \quad(f, \beta) \in \mathcal{D}_{\theta, 0}^{r+1}(S)\right\} .
\end{aligned}
$$

Recall our assumptions, $\left(M^{n}, g\right)$ with $(I),\left(B_{k+2}\right), k \geq r+1>\frac{2 n-1}{2}+2$. This implies $\omega \in{ }^{b, r+1} \Omega^{2}\left(T^{*} M\right)$. Additionally we have in the case of $T^{*} M$ that $\omega$ is strongly nondegenerate, i. e. $\inf _{z \in T^{*} M}|\omega|_{z}^{2}>0$.

It follows immediately from the definition (4.5) that $\tilde{f}$ is a $C^{2}$ diffeomorphism. Thus we get a 1-1 mapping between $\mathcal{D}_{\theta, 0}^{r+1}(S)$ and $\mathcal{D}_{\theta, 0}^{r+1}\left(T^{*} M \backslash 0\right)$. We endow $\mathcal{D}_{\theta, 0}^{r+1}\left(T^{*} M \backslash 0\right)$ with the topology and differential structure of $\mathcal{D}_{\theta, 0}^{r+1}(S)$ such that $\Phi$ becomes a diffeomorphism. Evidently, $\Phi(i d, 1)=i d_{T^{*} M \backslash 0}$. Our next aim is to describe properties of $\Phi$, of the elements of $\mathcal{D}_{\theta, 0}^{r+1}\left(T^{*} M \backslash 0\right)$ and of $T_{i d} \mathcal{D}_{\theta, 0}^{r+1}\left(T^{*} M \backslash 0\right)$.

\section{Proposition 4.7.}

a) $\Phi$ is an isomorphism of groups.

b) Each $\tilde{f} \in \mathcal{D}_{\theta, 0}^{r+1}\left(T^{*} M \backslash 0\right)$ satisfies $\tilde{f}^{*} \theta=\theta$.

c) Let $(X, h) \in T_{(i d, 1)} \mathcal{D}_{\theta, 0}^{r+1}(S)$. Then $\Phi_{*(i d, 1)}(X, u)=X_{H}$ where $H$ is the Hamiltonian $H(z)=\theta_{S}\left(X_{\frac{z}{|z|}}\right) \cdot|z|$, i.e.,

$$
H=\theta_{S}(X \circ \pi) / f_{S}, \quad f_{S}(z)=\frac{1}{|z|} .
$$

In particular, $H$ is homogeneous of degree one, $X_{H}$ is homogeneous of degree 0 and $H=\theta\left(X_{H}\right)$.

d) If $Y=Y_{H} \in T_{i d} \mathcal{D}_{\theta, 0}^{r+1}\left(T^{*} M \backslash 0\right)$ then $Y$ projects to $X=\pi_{*} Y$ tangentially to $S$ and $\Phi_{*}^{-1}(Y) \equiv\left(\Phi^{-1}\right)_{*}(Y)=\left(\pi_{*} Y, u\right)$, where $u\left(\frac{z}{|z|}\right)=$ $\left\{\frac{1}{|z|}, H(z)\right\} \cdot|z|$, i.e.,

$$
u \circ \pi=\left\{f_{S}, H\right\} / f_{S}
$$

Here $\{$,$\} the Poisson bracket on T^{*} M$. 
e) $\Phi_{*}$ preserves the Lie brackets.

f) $T_{i d} \mathcal{D}_{\theta, 0}^{r+1}\left(T^{*} M \backslash 0\right)$ coincides with the space

$$
\begin{aligned}
& \mathcal{H}_{\theta}^{0} \Omega^{0,2, r+1}\left(T\left(T^{*} M \backslash 0\right)\right) \\
& =\left\{Y \mid Y \text { is a } C^{2} \text { vector field on } T^{*} M \backslash 0, L_{Y} \theta=0\right. \\
& \left.\quad \text { and }\left.Y\right|_{S} \in \Omega^{0,2, r+1}\left(i^{*}\left(T\left(T^{*} M \backslash 0\right)\right)\right), \quad i: S \rightarrow T^{*} M \backslash 0\right\} .
\end{aligned}
$$

g) $\mathcal{H}_{\theta}^{0} \Omega^{0,2, r+1}\left(T\left(T^{*} M \backslash 0\right)\right)$ is isomorphic to the space

$$
\begin{aligned}
\mathcal{H}^{1} \Omega^{0,2, r+2}\left(T^{*} M \backslash 0\right)= & \left\{h \mid h \text { is a } C^{3} \text { function on } T^{*} M \backslash 0,\right. \\
& h \text { is homogeneous of degree } 1 \text { and } \\
& \left.i^{*} h=\left.h\right|_{S} \in \Omega^{0,2, r+2}(S)\right\} .
\end{aligned}
$$

h) $\mathcal{H}^{1} \Omega^{0,2, r+2}\left(T^{*} M \backslash 0\right)$ is isomorphic to $\Omega^{0,2, r+2}(S)$.

Proof. For a) we refer to [18], p. 97. Let $\tilde{f} \in \mathcal{D}_{\theta, 0}^{r+1}\left(T^{*} M \backslash 0\right)$. Recall $\theta_{S}=\left.\theta\right|_{S}=i^{*} \theta, \theta$ on $T^{*} M \backslash 0$ the canonical one form, $\pi: T^{*} M \backslash 0 \rightarrow$ $S\left(T^{*} M\right)=S$ the projection, $\pi(z)=\frac{z}{|z|}$. Then $\tilde{f}^{*} \theta=\left(\frac{i \circ f \circ \pi}{(\beta \circ \pi) \cdot f_{S}}\right)^{*} \theta=$ $\frac{\pi^{*} f^{*} \theta_{S}}{(\beta \circ \pi) \cdot f_{S}}=\frac{(\beta \circ \pi) \pi^{*} \theta_{S}}{(\beta \circ \pi) f_{S}}=\theta$. This proves b). We conclude, according to 4.5.b) that $\tilde{f}$ is symplectic and homogeneous of degree one. Claims c), d) and e) are again simple calculations, performed in [18], pp. 97, 98. For claim f) we use the fact that $X_{H}$ is homogeneous of degree 0 to assure that $Y_{H}$ projects to $\pi_{*} Y_{H}$ tangentially to $S$. Let $Y=X_{H}=\Phi_{*}(X, u) \in T_{i d} \mathcal{D}_{\theta, 0}^{r+1}\left(T^{*} M \backslash 0\right) \equiv$ $\Phi_{*} T_{i d} \mathcal{D}_{\theta, 0}^{r+1}(S)$. Assuming for a moment $Y=X_{H} \in C^{2}$, we conclude from 4.6.c) that $L_{X} \theta=0$. Hence we have only to show that $Y=X_{H}$ is $C^{2}$ and $\left.Y\right|_{S} \in \Omega^{0,2, r+1}\left(i^{*}\left(T\left(T^{*} M \backslash 0\right)\right)\right)$. The latter would imply that $\left.Y\right|_{S} \in C^{2}$ (according to the Sobolev embedding theorem), hence $Y \in C^{2}$ since $Y$ is homogeneous of degree zero. Assuming $\left.Y\right|_{S}=\left.X_{H}\right|_{S} \in \Omega^{0,2, r+1}\left(i^{*}\left(T\left(T^{*} M \backslash\right.\right.\right.$ $0))$ ), we have $T_{i d} \mathcal{D}_{0}^{r+1}\left(T^{*} M \backslash 0\right) \subseteq \mathcal{H}_{\theta}^{0} \Omega^{0,2, r+1}\left(T\left(T^{*} M \backslash 0\right)\right)$. Consider $\supseteq$. Let $Y \in \mathcal{H}_{\theta}^{0} \Omega^{0,2, r+1}\left(T\left(T^{*} M \backslash 0\right)\right)$. Then, according to 4.6.c) $Y=Y_{H}$ for some $H$ and $Y$ has degree zero homogeneity. Hence it projects to $T S$, $\pi_{*} Y=X$. Define $u$ by (4.8). Assuming for a moment $X \in \Omega^{0,2, r+1}(T S)$ and $u \in \Omega^{0,2, r+1}(S)$, we see by an easy calculation $\Phi_{*}(X, u)=Y_{H}$, i.e., $\supseteq$ would be proved. Hence there remains to show

1. $Y=X_{H} \in T_{i d} \mathcal{D}_{\theta, 0}^{r+1}\left(T^{*} M \backslash 0\right)$ implies $\left.Y\right|_{S} \in \Omega^{0,2, r+1}\left(i^{*}\left(T\left(T^{*} M \backslash 0\right)\right)\right)$, 
2. $Y \in \mathcal{H}_{\theta}^{0} \Omega^{0,2, r+1}, Y=Y_{H}$ then $X=\pi_{*} Y \in \Omega^{0,2, r+1}(T S)$ and $u \in$ $\Omega^{0,2, r+1}(S)$.

Lets begin with the first assertion $Y=X_{H}=\Phi_{*}(X, u)$. We introduce local coordinates in $T\left(T^{*} M \backslash 0\right)$, say $\left(x, \xi, \psi_{1}, \psi_{2}\right), x$ coordinates im $M, \xi$ in $T^{*} M \backslash 0, \psi_{1}, \psi_{2}$ over them in $T\left(T^{*} M \backslash 0\right)$ with projections $\pi_{1}, \pi_{2}$. Then according to [18], p. 99 we have

$$
\left.X_{H}\right|_{S}(x, \xi)=\left.\Phi_{*}(X, u)\right|_{S}(x, \xi)=\left(x, \xi,(X)_{1}(x, \xi),(X)_{2}(x, \xi)-u(x, \xi) \cdot \xi\right)
$$

where ()$_{i}$ are the components of $X$ belonging to $i m \pi_{i}$. Using a uniformly locally finite cover of $S\left(T^{*} M \backslash 0\right),(4.9)$ and $X \in \Omega^{0,2, r+1}(T S), u \in \Omega^{0,2, r+1}(S)$, we conclude that $\left.Y\right|_{S}=\left.X_{H}\right|_{S} \in \Omega^{0,2, r+1}\left(i^{*}\left(T\left(T^{*} M \backslash 0\right)\right)\right)$.

To prove 2., we assume $Y=Y_{H} \in \mathcal{H}_{\theta}^{0} \Omega^{0,2, r+1}\left(T\left(T^{*} M \backslash 0\right)\right)$. By assumption $\left.Y\right|_{S} \in \Omega^{0,2, r+1}\left(i^{*}\left(T\left(T^{*} M \backslash 0\right)\right)\right)$. Set $X=\pi_{*} Y=\left.\pi_{*} Y\right|_{S}$. Denote by $\nabla^{S}$ the Levi-Civita connection of $\left(S\left(T^{*} M\right), g_{S}\right)$. Then by choice of local orthonormal bases $e_{1}, \ldots, e_{2 n-1}, e_{2 n}, e_{2 n} \perp S$, we see immediately for $i \leq r+1$

$$
|X|_{g_{S}} \leq|Y|_{S}|, \quad|\left(\nabla^{S}\right)^{i} X|\leq| \nabla^{i}\left(\left.Y\right|_{S}\right) \mid
$$

which implies $X \in \Omega^{0,2, r+1}(T S)$. Write as in (4.9)

$$
X(x, \xi)=\left(x, \xi,(X)_{1}(x, \xi),(X)_{2}(x, \xi)\right)
$$

Then, locally,

$$
\left(\left.X_{H}\right|_{S}-X\right)(x, \xi)=(0,0,0,-u(x, \xi) \cdot \xi)
$$

which immediately implies $u \in \Omega^{0,2, r+1}(S)$.

This finishes the proof of $\mathrm{f}$ ).

Consider g) and the map $Y=X_{H} \mapsto H=\theta\left(X_{H}\right)$ given by 4.6.c. We must prove that $H \in C^{3}$ and $\left.H\right|_{S}=i^{*} H \in \Omega^{0,2, r+2}(S)$. The latter will already imply $H \in C^{3}$. We immediately obtain from (4.7) that $\left.H\right|_{S} \in$ $\Omega^{0,2, r+1}(S)$ since $H=\theta\left(X_{H}\right),\left.H\right|_{S}=\theta\left(\left.X_{H}\right|_{S}\right), \theta \in \in^{b, r+1} \Omega^{1}$ and $\left.X_{H}\right|_{S} \in$ $\Omega^{0,2, r+1}(T S)$. Hence $H \in C^{2}$. The main point is that $\left.H\right|_{S}$ has even Sobolev order $r+2$. Denote again by $d_{S}, \nabla^{S}$ the operators $d, \nabla$ on $S$. We have

$$
\left|d_{S}\left(\left.H\right|_{S}\right)\right| \leq\left.|(d H)|_{S}|,|\left(\nabla^{S}\right)^{i}\left(\left.H\right|_{S}\right)|\leq|\left(\nabla^{i} H\right)\right|_{S} \mid .
$$

For nonsmooth objects we have (as usual) to understand this in the distributional sense. We always have to do with regular distributions. That $\left.H\right|_{S} \in \Omega^{0,2, r+2}(S)$ would be proved if we could show that 
$\left.d_{S} H\right|_{S} \in \Omega^{1,2, r+1}(S)$. According to (4.13) this would be done if we could show $\left.(d H)\right|_{S},\left.\left.(\nabla d H)\right|_{S} \ldots\left(\nabla^{r+1} d H\right)\right|_{S}$ are square integrable on $S$. But $\left.(d H)\right|_{S}=\left.\left(i_{X_{H}} \omega\right)\right|_{S}=i_{\left.X_{H}\right|_{S}} \omega$. Furthermore $\omega \in \epsilon^{b, r+2} \Omega^{2}, \omega$ is strongly nondegenerate and $\left.X_{H}\right|_{S} \in \Omega^{0,2, r+1}\left(i^{*}\left(T\left(T^{*} M \backslash 0\right)\right)\right)$ just imply that $\left.(d H)\right|_{S}, \ldots,\left.\left(\nabla^{r+1} d H\right)\right|_{S}$ are square integrable on $M$. This is Lemma 3.6, 3.7 in [15]. We obtained that $H \in \mathcal{H}^{1} \Omega^{0,2, r+2}\left(T^{*} M \backslash 0\right)$, and from 4.6 it follows that the map $X_{H} \mapsto \theta\left(X_{H}\right) \mapsto X_{\theta\left(X_{H}\right)}$ equals to the identity.

Now let $h \in \mathcal{H}^{1} \Omega^{0,2, r+2}\left(T^{*} M \backslash 0\right)$. The function $h$ defines a global Hamiltonian vector field $X_{h}$, homogeneous of degree zero, satisfying $L_{X_{h}} \theta=$ 0 . Moreover $X_{h}$ is $C^{2}$ and solves the equation $i_{X_{h}} \omega=d h$. We have to assure that $\left.X_{h}\right|_{S} \in \Omega^{0,2, r+1}\left(i^{*}\left(T\left(T^{*} M \backslash 0\right)\right)\right)$. From $\left.h\right|_{S} \in \Omega^{0,2, r+2}(S)$ we conclude that $|h|_{S},\left|d_{S} h\right|$ and $\left|\left(\nabla^{S}\right)^{i} d_{S} h\right|$ are square integrable on $S$. But for $i \geq 1$

$$
\left|\left(\nabla^{i} h\right)\right|_{S} \leq\left|\left(\nabla^{S}\right)^{i-1}\left(\left.h\right|_{S}\right)\right|+\left|\left(\nabla^{S}\right)^{i}\left(\left.h\right|_{S}\right)\right|,
$$

since $h$ is homogeneous of degree one. We obtain that $|(d h)|_{S}, \ldots,\left|\left(\nabla^{r+1} d h\right)\right|_{S}$ are square integrable on $S$, i.e., the right hand side of $i_{X_{h}} \omega_{S}=\left.d h\right|_{S}$ is an element of $\Omega^{1,2, r+1}(S)$ (with values in the conormal bundle of $S$ ). Then $X_{h} \in \Omega^{0,2, r+1}\left(i^{*}\left(T\left(T^{*} M \backslash 0\right)\right)\right)$ and altogether $X_{h} \in \mathcal{H}_{\theta}^{0} \Omega^{0,2, r+1}\left(T\left(T^{*} M \backslash 0\right)\right)$. According to 4.6, the map $h \mapsto X_{h} \mapsto \theta\left(X_{h}\right)$ coincides with $i d$. This finishes th proof of claim g).

Concerning claim $\mathrm{h}$ ), the isomorphism is given by $h \in \mathcal{H}^{1} \Omega^{0,2, r+2}\left(T^{*} M \backslash\right.$ $0)\left.\mapsto h\right|_{S}$. This map is well defined, according to g). It is injective since $h$ is homogeneous of degree one. It is surjective because for $u \in \Omega^{0,2, r+2}(S)$ let $h_{u}$ be its extension homogeneous of degree one. Then $h_{u} \in \mathcal{H}^{1} \Omega^{0,2, r+2}\left(T^{*} M \backslash 0\right)$ and $\left.h_{u}\right|_{S}=u$.

Remark. We constructed a topological isomorphism

$$
F: \mathbf{d}_{\theta, 0}^{r+1}(S) \longrightarrow \Omega^{0,2, r+2}(S) .
$$

This isomorphism is topological since all constructed maps in Proposition 4.7 are norm continuous. Here we essentially use Lemma 3.6, 3.7 of [15]. The isomorphism (4.14) will be very important in constructing local charts on $\mathcal{D}_{\theta, 0}^{r+1}(S)$. Proposition 4.7. justifies to denote $\mathcal{D}_{\theta, 0}^{r+1}(S)$ and $\mathcal{D}_{\theta, 0}^{r+1}\left(T^{*} M \backslash 0\right)$ by the same symbol. We can understand $\mathcal{D}_{\theta, 0}^{r+1}\left(T^{*} M \backslash 0\right)$ as a Hilbert manifold and a topological group with $T_{i d} \mathcal{D}_{\theta, 0}^{r+1}\left(T^{*} M \backslash 0\right) \cong \Omega^{0,2, r+2}(S)$.

Summarizing our results, we obtain in the case of $\left(B_{\infty}\right)$ the following:

Theorem 4.8. Suppose $\left(M^{n}, g\right)$ with $(I),\left(B_{\infty}\right)$ and

$$
\inf \sigma_{e}\left(\left.\triangle_{1}\left(g_{S}\right)\right|_{\text {ker } \triangle_{1}\left(g_{S}\right)^{\perp}}\right)>0 .
$$


Set $\mathcal{D}_{\theta, 0}^{\infty}\left(T^{*} M \backslash 0\right)=\lim _{\leftarrow} \mathcal{D}_{\theta, 0}^{r+1}\left(T^{*} M \backslash 0\right)$. Then

$$
\left\{\mathcal{D}_{\theta, 0}^{\infty}\left(T^{*} M \backslash 0\right), \mathcal{D}_{\theta, 0}^{r+1}\left(T^{*} M \backslash 0\right) \mid r+1>\frac{2 n-2}{2}+2\right\}
$$

is an ILH Lie group.

\section{Pseudodifferential and Fourier Integral Operators on Open Manifolds.}

Pseudodifferential ( $\Psi D O)$ and Fourier integral operators (FIO) are well defined for any manifold, open or closed. But on open manifolds the spaces of these operators don't have any reasonable structure. Moreover, many theorems for $\Psi$ DOs or FIOs on closed manifolds become wrong or don't make any sense in the open case, e.g., certain mapping properties between Sobolev spaces of functions are wrong. The situation rapidly changes if we restrict ourselves to bounded geometry and adapt these operators to the bounded geometry. This means, roughly speaking, that the family of local symbols together with their derivatives should be uniformly bounded. For FIOs we additionally restrict ourselves to comparatively smooth Lagrangian submanifolds $\Lambda$ of $T^{*} M \backslash 0 \times T^{*} M \backslash 0$ and phase functions also adapted to the bounded geometry.

A good reference for $\Psi D O$ 's are [16] and [20]. Further results are in preparation (cf. [14]). Since we restrict our applications to the case where the Riemannian manifold $\left(M^{n}, g\right)$ satisfies the conditions of bounded geometry $(I)$ and $\left(B_{\infty}\right)$, we assume these conditions from now on. Moreover, we restrict ourselves to the scalar case, i.e., we consider only operators acting on functions.

We first recall two classical lemmas which play a key role in all forthcoming constructions.

Lemma 5.1. Assume $\left(M^{n}, g\right)$ with $(I)$ and $\left(B_{\infty}\right)\left(\left(B_{0}\right)\right.$ is sufficient here $)$, $\delta<\frac{r_{i n j}}{2}$. Then there exists a uniformly locally finite cover $\mathcal{U}=\left\{U_{i}\right\}_{i}$ of $M$ by geodesic $\delta$-balls.

Lemma 5.2. Assume $\mathcal{U}=\left\{U_{i}\right\}_{i}$ as above (and $\left(B_{\infty}\right)$ ). Then there exists an associated partition of unity $\left\{\psi_{i}\right\}_{i}$ such that

$$
\left|\nabla^{k} \psi_{i}\right| \leq C_{k}, \quad k=0,1,2, \ldots,
$$


We define now $U \Psi^{-\infty}(M)$ to be the set of all linear operators $R$ : $C_{c}^{\infty}(M) \rightarrow C_{c}^{\infty}(M)$ which have the following properties.

(5.2) 1. $R$ has Schwartz kernel $\mathcal{K}_{R} \in C^{\infty}(M \times M)$.

(5.3) 2. There exists a constant $C_{R}$ s. t. $\mathcal{K}_{R}(x, y)=0$ for $d(x, y)>C_{R}$.

(5.4) 3. $\nabla_{x}^{i} \nabla_{y}^{j} \mathcal{K}_{R}$ is bounded for all $i$ and $j$.

It follows from the conditions $(I)$ and $\left(B_{\infty}\right)$ that for any point $m \in M^{n}$ there exists a diffeomorphism $\Phi_{m}$

$$
M \supset B_{\varepsilon}(m) \stackrel{\Phi_{m}}{\longrightarrow} B=B_{\varepsilon}(0) \subset \mathbf{R}^{n}
$$

such that $\Phi_{m}$ induces bounded isomorphisms

$$
\begin{gathered}
{ }^{b, k} \Omega^{0}\left(B_{\varepsilon}(m)\right) \stackrel{\cong}{b, k} \Omega^{0}(B), \\
{ }^{b, \infty} \Omega^{0}\left(B_{\varepsilon}(m)\right) \stackrel{\cong}{b, \infty} \Omega^{0}(B)
\end{gathered}
$$

with bounds independent of $m$. After fixing an orthonormal basis in $T_{m} M$, $\Phi_{m}$ is essentially given by the exponential map.

We now define the class of uniform symbols for our pseudodifferential operators as follows. Let $q \in \mathbf{R}$ and denote by $\mathcal{U} S^{q}(B)$ the set of all families $\left\{a_{m}\right\}_{m \in M}$ with $a_{m} \in C^{\infty}\left(B \times \mathbf{R}^{n}\right)$ and

$$
\left|\partial_{\xi}^{\alpha} \partial_{x}^{\beta} a_{m}(x, \xi)\right| \leq C_{\alpha, \beta}(1+|\xi|)^{q-|\alpha|},
$$

where $C_{\alpha, \beta}$ is independent of $m$. Then $\left\{a_{m}\right\}_{m}$ defines a family of operators

$$
a_{m}\left(x, D_{x}\right): C_{c}^{\infty}(B) \longrightarrow C^{\infty}(B)
$$

by

$$
a_{m}\left(x, D_{x}\right) u(x):=(2 \pi)^{-n} \int_{\mathbf{R}^{n}} \int_{B} a_{m}(x, \xi) u(y) d y d \xi,
$$

where supp $u \subset B$.

Define $\mathcal{U} \Psi^{-\infty}(B)$ as the set of all families $\left\{R_{m}: C_{c}^{\infty}(B) \rightarrow C^{\infty}(B)\right\}_{m \in M}$ such that $R_{m}$ has Schwartz kernel $\mathcal{K} R_{m} \in C^{\infty}(B \times B)$ with

$$
\left|\partial_{x}^{\alpha} \partial_{y}^{\beta} \mathcal{K}_{R_{m}}(x, y)\right| \leq C_{\alpha, \beta},
$$


where $C_{\alpha, \beta}$ is independent of $m$. Finally let $\mathcal{U} \Psi^{q}(B)$ be the set of all families $\left\{A_{m}: C_{c}^{\infty}(B) \rightarrow C^{\infty}(B)\right\}_{m \in M}$ such that

$$
A_{m}=a_{m}\left(x, D_{x}\right)+R_{m},
$$

$\left\{a_{m}\right\}_{m} \in \mathcal{U} S^{q}(B),\left\{R_{m}\right\}_{m} \in \mathcal{U} \Psi^{-\infty}(B)$.

Now we define the space $\mathcal{U} \Psi^{q}(M)$ of uniform pseudodifferential operators of order $q$ on $M^{n}$ as follows: A pseudodifferential operator $A$ on $M^{n}$ with Schwartz kernel $\mathcal{K}_{A}$ belongs to $\mathcal{U} \Psi^{q}(M)$ iff it satisfies the following conditions:

1. There exists a constant $C_{A}>0$ s. t.

$$
\mathcal{K}_{A}(x, y)=0 \text { for } d(x, y)>C_{A}, x, y \in M .
$$

2. $\mathcal{K}_{A}$ is smooth outside the diagonal of $M \times M$.

3. For any $\delta>0$ and $i, j$ there exists a constant $C_{\delta, i, j}>0$ s. t.

$$
\left|\nabla_{x}^{i} \nabla_{y}^{j} \mathcal{K}_{A}(x, y)\right| \leq C_{\delta, i, j} \text { for } d(x, y)>\delta .
$$

4. If $A_{m}$ is defined by the following commutative diagram

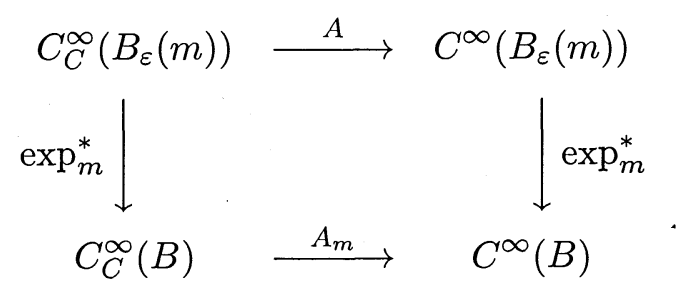

then the family $\left\{A_{m}\right\}_{m \in M}$ belongs to $\mathcal{U} \Psi^{q}(B)$.

Remark. We have $\mathcal{U} \Psi^{-\infty}(M)=\bigcap_{q} \mathcal{U} \Psi^{q}(M)$.

A convenient description for the elements $A \in \mathcal{U} \Psi^{q}(M)$ is given by

Proposition 5.3. Assume $A \in \mathcal{U} \Psi^{q}(M)$, and $\varepsilon>0$ arbitrary. Then there exists a representation $A=A_{1}+A_{2}$
a. $A_{1} \in \mathcal{U} \Psi^{-\infty}(M)$
b. $A_{2} \in \mathcal{U} \Psi^{q}(M)$ 
c. $\mathcal{K}_{A_{2}(x, y)}=0$ for $d(x, y)>\varepsilon$

i.e., up to smoothing operators in $\mathcal{U} \Psi^{-\infty}(M)$ we can always assume that the support of $\mathcal{K}_{A}$ is arbitrary dense to the diagonal.

We refer to [16], p. 230/231 for the proof.

For our applications we additionally restrict ourselves to classical symbols and classical $\Psi D O$ s, i.e., we assume homogeneity in the $\xi$-variable on $\mathbf{R}^{n} \backslash\{0\}$ and an asymptotic expansion

$$
a_{m}(x, \xi) \sim \sum_{j=0}^{\infty} a_{m, q-j}(x, \xi)
$$

such that $a_{m, q-j}(x, \xi)$ is positive homogeneous of degree $q-j$ in $\xi$. Here $a_{m}(x, \xi) \sim \sum_{j=0}^{\infty} a_{m, q-j}(x, \xi)$ means

$$
\left\{(1-\chi(x, \xi))\left(a_{m}(x, \xi)-\sum_{j=1}^{k-1} a_{m, q-j}(x, \xi)\right)\right\}_{m} \in \mathcal{U} S^{q-k}(B)
$$

for all $k$ and $\chi(x, \xi)$ is compactly supported in the $\xi$-direction with $\chi=1$ in a neighborhood of $B \times\{0\}$.

Remark. Shubin [20] calls such pseudodifferential operators $\Psi$ DOs with polyhomogeneous local symbols and writes $\mathcal{U} \Psi_{p h g}^{q}(M)$ but we omit the subscript phg and write simply $\mathcal{U} \Psi^{q}(M)$.

We recall some mapping properties and refer to [16] for the proofs.

Proposition 5.4. Any $R \in \mathcal{U} \Psi^{-\infty}(M)$ defines continuous maps and extensions as follows

$$
\begin{aligned}
& R: C_{c}^{\infty}(M) \longrightarrow C_{c}^{\infty}(M), \\
& R: C^{\infty}(M) \longrightarrow C^{\infty}(M), \\
& R: \mathcal{E}^{\prime} \longrightarrow C_{c}^{\infty}(M) \\
& R:{ }^{b, \infty} \Omega^{0}(M) \longrightarrow{ }^{b, \infty} \Omega^{0}(M) .
\end{aligned}
$$

Proposition 5.5. $A \in \mathcal{U} \Psi^{q}(M)$ defines linear continuous maps

$$
A: \Omega^{0,2, r} \longrightarrow \Omega^{0,2, r-q}
$$

and

$$
A:{ }^{b, \infty} \Omega^{0} \longrightarrow{ }^{b, \infty} \Omega^{0}
$$


Finally we have

\section{Proposition 5.6.}

a. If $R_{1}, R_{2} \in \mathcal{U} \Psi^{-\infty}(M)$ then $R_{1} \circ R_{2} \in \mathcal{U} \Psi^{-\infty}(M)$.

b. If $R \in \mathcal{U} \Psi^{-\infty}(M)$ then $R^{*} \in \mathcal{U} \Psi^{-\infty}(M)$.

c. If $A \in \mathcal{U} \Psi^{q}(M)$ then $A^{*} \in \mathcal{U} \Psi^{q}(M)$

d. If $A \in \mathcal{U} \Psi^{q_{1}}(M), B \in \mathcal{U} \Psi^{q_{2}}(M)$ then $A \circ B \in \mathcal{U} \Psi^{q_{1}+q_{2}}(M)$.

All proofs are performed locally. Using the uniform boundedness (5.5), one gets these results for the formulas of the symbols of the adjoint operators and the product (composition) of operators.

Finally we recall uniform ellipticity. $A \in \mathcal{U} \Psi^{q}(M)$ is called uniformly elliptic if there exist constants $C_{1}, C_{2}, R>0$, independent of $m \in M$, such that

$$
C_{1}|\xi|^{q} \leq\left|a_{m}(x, \xi)\right| \leq C_{2}|\xi|^{q}
$$

for all $|\xi|>R, x \in B, m \in M$. We denote this class of operators by $E \mathcal{U} \Psi^{q}(M)$.

Remark. Given any real number $s$, then there exists a uniformly elliptic operator in $E \mathcal{U} \Psi^{s}(M)$, e.g., $(1+\triangle)^{\frac{s}{2}} \in E \mathcal{U} \Psi^{s}(M)$.

Theorem 5.8. Given $A \in E \mathcal{U} \Psi^{q}(M)$, then there exists a parametrix, i.e., a $P \in \mathcal{U} \Psi^{-q}(M)$ s. $t$.

$$
P \circ A=I+R_{1}, \quad A \circ P=I+R_{2}, \quad R_{1}, R_{2} \in \mathcal{U} \Psi^{-\infty}(M) .
$$

The proof is performed locally by establishing explicit formulas for the symbol $\left\{p_{m}\right\}_{m}$. This is done as usual by calculation of the terms of the asymptotic expansion (5.10). Then one fits the local operators together by a partition of unity. To assure $P \in \mathcal{U} \Psi^{-q}(M)$, one essentially uses (5.1).

Remark. In contrast to the case of compact manifolds, (5.12) does not mean the invertibility of $A$ modulo compact operators. On open manifolds, the kernels $\mathcal{K}_{R_{1}}, \mathcal{K}_{R_{2}}$ are far from being square integrable, i.e., $R_{1}, R_{2}$ are far from being compact operators. As a simple consequence, $P$ is far from being Fredholm (except in very special cases). 
Taking 5.3 into account, we define as in [1] a formal $\Psi D O$ of order $q$ as an element of $\mathcal{U} \Psi^{q}(M) / \mathcal{U} \Psi^{-\infty}(M)$. Denote by $(\mathcal{U} \Psi(M))_{*}$ the set of all invertible elements in $\mathcal{U} \Psi(M)$,

$$
\mathcal{U} \Psi(M):=\bigcup_{q} \mathcal{U} \Psi^{q}(M) / \mathcal{U} \Psi^{-\infty}(M)=\left(\bigcup_{q} \mathcal{U} \Psi^{q}(M)\right) / \mathcal{U} \Psi^{-\infty}(M) .
$$

$(\mathcal{U} \Psi(M))_{*}$ is a graded group under multiplication and non-empty as 5.8 shows.

Quite similarly we define uniform Fourier integral operators $\mathcal{U} F^{q}(M, C)$. A Fourier integral operator (FIO) on $M$ has essentially 3 ingredients

1. a family $a=\left\{a_{m}\right\}_{m}$ of local uniform symbols as above,

2. a conic Lagrangian submanifold (or homogeneous canonical relation) $C \subset T^{*} M \backslash 0 \times T^{*} M \backslash 0$,

3. a family $\varphi=\left\{\varphi_{m}\right\}_{m \in M}$ of phase functions.

We will make this precise. Recall that we now always assume that $\left(M^{n}, g\right)$ satisfies the conditions $(I)$ and $\left(B_{\infty}\right)$. A homogeneous canonical relation $C$ is a closed submanifold (not in the sense of compactness) $C \subset T^{*} M \backslash 0 \times T^{*} M \backslash 0$ which is conical, i.e., $((x, \xi),(y, \eta) \in C$ and $\tau>0$ imply $((x, \tau \xi),(y, \tau \eta) \in C$, and which is Lagrangian with respect to the canonical symplectic form $\omega \ominus \omega=p_{1}^{*} \omega-p_{2}^{*} \omega$, where $p_{j}: T^{*} M \times T^{*} M \longrightarrow T^{*} M, j=1,2$ are the projections and $\omega=-d \theta$. A very important class of examples are the graphs $\Gamma(\tilde{f})$ of contact transformations. Let $f \in \mathcal{D}_{\theta, 0}^{r+1}\left(S\left(T^{*} M\right)\right)$ and $\tilde{f}=\Phi(f)$ the corresponding homogeneous diffeomorphism $\tilde{f}: T^{*} M \backslash 0 \longrightarrow T^{*} M \backslash 0$ satisfying $\tilde{f}^{*} \theta=\theta$, which is given by $(4.5)$. Then $\Gamma(\tilde{f})=\{((x, \xi),(y, \eta)) \mid \tilde{f}(x, \xi)=(y, \eta)\}$ is conical and Lagrangian according to Lemma 4.5.

Uniform families $a=\left\{a_{m}\right\}_{m \in M}$ of local symbols are already defined but we consider here a slight generalization of (5.5) admitting additional dependence of a second variable $y$, i.e., we require

$$
\left|\partial_{\xi}^{\alpha} \partial_{x}^{\beta} \partial_{y}^{\gamma} a_{m}(x, y, \xi)\right| \leq C_{\alpha, \beta, \gamma}(1+|\xi|)^{q-|\alpha|},
$$

where $C_{\alpha, \beta, \gamma}$ is independent of $m$. We write $\mathcal{U} S^{q}\left(B \times B \times \mathbf{R}^{n} \backslash 0\right)$ for all such symbols $a=\left\{a_{m}\right\}_{m}$. Consider $\varphi=\left\{\varphi_{m}\right\}_{m \in M}$ with the following properties. Each $\varphi_{m}: B \times B \times \mathbf{R}^{n} \backslash 0 \longrightarrow \mathbf{R}$ is a smooth map, positive homogeneous of degree one with respect to $\xi \in \mathbf{R}^{n} \backslash 0$, i.e., $\varphi_{m}(x, y, \tau \xi)=\tau \cdot \varphi_{m}(x, y, \xi)$ 
and $d_{x \xi} \varphi_{m}, d_{y \xi} \varphi_{m}$ are $\neq 0$ on the canonical support of $a_{m}$. Furthermore, the map

$$
\begin{array}{r}
\left\{(x, y, \xi) \in \text { canonical support of } a_{m} \mid d_{\xi} \varphi_{m}(x, y, \xi)=0\right\} \\
\longmapsto \\
\left\{\left(x, d_{x} \varphi_{m}(x, y, \xi), y,-d_{y} \varphi_{m}(x, y, \xi)\right) \mid(x, y, \xi) \text { as above }\right\}
\end{array}
$$

is a diffeomorphism onto a conical submanifold $C_{B} \subset T^{*} B \backslash 0 \times T^{*} B \backslash 0$, where $C_{B}$ corresponds to $C \subset T^{*} M \times T^{*} M$ under the exponential map.

Such a family $\varphi=\left\{\varphi_{m}\right\}_{m \in M}$ is called a uniform family of phase functions associated to $a=\left\{a_{m}\right\}$ and we write $\mathcal{U} P h(a)\left(B \times B \times \mathbf{R}^{n}\right)$ for the space of all such families. We say $A$ is a uniform Fourier integral operator of order $q$, associated to the homogeneous canonical relation $C \subset T^{*} M \backslash 0 \times T^{*} M \backslash 0$, written as $A \in \mathcal{U} F^{q}(M, C)$, if it satisfies the following conditions:

1. $A$ is a continuous linear map $A: C_{c}^{\infty}(M) \longrightarrow C^{\infty}(M)$,

2. If the family $\left\{A_{m}\right\}_{m \in M}$ is defined by the commutative diagram

$$
\begin{array}{ccc}
C_{c}^{\infty}\left(B_{\varepsilon}(m)\right) & \stackrel{A}{\longrightarrow} & C^{\infty}\left(B_{\varepsilon}(m)\right) \\
\exp _{m}^{*} \downarrow & & \downarrow \exp _{m}^{*} \\
C_{c}^{\infty}(B) & \stackrel{A_{m}}{\longrightarrow} & C^{\infty}(B)
\end{array}
$$

then there exist $a=\left\{a_{m}\right\}_{m} \in \mathcal{U} S^{q}\left(B \times B \times \mathbf{R}^{n} \backslash 0\right)$ such that

$$
A_{m} u(x)=(2 \pi)^{-n} \int_{\mathbf{R}^{n}} \int_{B} e^{i \varphi_{m}(x, y, \xi)} a_{m}(x, y, \xi) u(y) d y d \xi,
$$

supp $u \subset B$, and for $\psi:=\left(\exp _{m}^{*}\right)^{-1} \varphi_{m}$ the map

$$
(x, y, \xi) \longmapsto\left(x, d_{x} \psi(x, y, \xi), y,-d_{y} \psi(x, y, \xi)\right)
$$

is a diffeomorphism from the zero set of $d_{\xi} \psi$ onto a submanifold of $C$.

Because of our applications, we restrict ourselves to the case where $C=\Gamma(\tilde{f}), f \in \mathcal{D}_{\theta, 0}^{r+1}\left(S\left(T^{*} M\right)\right)$, and we write $\operatorname{simply} \mathcal{U} F^{q}(f)$ for the corre- 
sponding class of uniform Fourier integral operators. We set

$$
\begin{aligned}
\mathcal{U} F^{-\infty}(f) & :=\bigcap_{q} \mathcal{U} F^{q}(f), \\
\mathcal{U} F^{q}(r+1) & :=\bigcup_{f \in \mathcal{D}_{\theta, 0}^{r+1}\left(S T^{*} M\right)} \mathcal{U} F^{q}(f), \\
\text { for }-k \leq q, \quad \mathcal{U} F^{q, k}(f) & :=\mathcal{U} F^{q}(f) / \mathcal{U} F^{-k-1}(f), \\
\mathcal{U} F^{q, k}(r+1) & :=\bigcup_{f \in \mathcal{D}_{\theta, 0}^{r+1}} \mathcal{U} F^{q, k}(f) .
\end{aligned}
$$

For $A_{1} \in \mathcal{U} F^{q_{1}}\left(f_{1}\right)$ and $A_{2} \in \mathcal{U} F^{q_{2}}\left(f_{2}\right)$ we have

$$
A_{1} \circ A_{2} \in \mathcal{U} F^{q_{1}+q_{2}}\left(f_{1} \circ f_{2}\right) .
$$

Denote analogously to (5.18) for $-k \leq q$

$$
\mathcal{U} \Psi^{q, k}:=\mathcal{U} \Psi^{q} / \mathcal{U} \Psi^{-k-1}
$$

and $\mathcal{D}_{\theta, 0}^{\infty}=\lim _{\leftarrow} \mathcal{D}_{\theta, 0}^{r+1}$,

$$
\begin{aligned}
\mathcal{U} F^{q, k}(\infty) & :=\lim _{\leftarrow} \mathcal{U} F^{q, k}(r+1)=\bigcup_{f \in \mathcal{D}_{\theta, 0}^{\infty}} \mathcal{U} F^{q, k}(f) . \\
\mathcal{U} S^{q, k} & :=\mathcal{U} S^{q} / \mathcal{U} S^{-k-1} .
\end{aligned}
$$

Let $\left(\mathcal{U} F^{0, k}(r+1)\right)_{*},\left(\mathcal{U} F^{0, k}(\infty)\right)_{*}$ and $\left(\mathcal{U} \Psi^{0, k}\right)_{*}$ denote the groups of invertible elements of $\mathcal{U} F^{0, k}(r+1), \mathcal{U} F^{0, k}(\infty)$ and $\mathcal{U} \Psi^{0, k}$ respectively. It is clear from (5.20) that we must choose $q=0$ to get invertibility inside one homogeneous constituent of our graded structures.

Lemma 5.9. Assume the hypothesizes of 4.8. The following is an exact sequence of groups

$$
I \longrightarrow\left(\mathcal{U} \Psi^{0, k}\right)_{*} \stackrel{j}{\longrightarrow}\left(\mathcal{U} F^{0, k}(\infty)\right)_{*} \stackrel{\pi}{\longrightarrow} \mathcal{D}_{\theta, 0}^{\infty} \longrightarrow e,
$$

where $j$ is the inclusion and $\pi[A]=f$, where $A \in \mathcal{U} F^{0}(f),[A]=A+$ $\mathcal{U} F^{-k-1}(f)$.

Proof. The injectivity of $j$, the surjectivity of $\pi$ and $i m j \subseteq k e r \pi$ are clear. $i m j=k e r \pi$ follows from (5.26).

Generalizing the ideas of [1] and [2] our strategy is as follows: 
1. We want to construct a local section of $\pi$ in (5.24). For this we need a chart in $\mathcal{D}_{\theta, 0}^{r+1}$ at $i d=e$.

2. This yields a chart and local section in (5.24),

3. We endow $\left(\mathcal{U} \Psi^{0, k}\right)_{*}$ with the structure of an ILH Lie group by forming Sobolev completions and taking the inverse limit.

4. We endow $\left(\mathcal{U} F^{0, k}(\infty)_{*}\right.$ with the structure of an ILH Lie group, using these structures of $\left(\mathcal{U} \Psi^{0, k}\right)_{*}$ and $\mathcal{D}_{\theta, 0}^{\infty}$ in (5.24), the local section of $\pi$ and some group theoretical theorems presented in Section 7 .

This procedure is carried out in [1], [2] for compact manifolds, but in the case of open manifolds the analysis is much harder. We first start with the construction of a local section of $\pi$ in (5.24). This means the existence of a neighborhood $\mathcal{U}(i d) \subset \mathcal{D}_{\theta, 0}^{r+1}$ and a (at least continuous) map $\sigma: \mathcal{U} \longrightarrow$ $\left(\mathcal{U} F^{0, k}(r+1)\right)_{*}$ such that $\pi \circ \sigma=i d_{\mathcal{U}}$. For doing this we construct global phase functions and present an explicit formula for $\sigma$.

We call a $C^{2}$ function $\varphi: T^{*} M \times M \rightarrow \mathbf{R}$ a global phase function for $e=i d \in \mathcal{D}_{\theta, 0}^{r+1}$ iff

1. $d \varphi\left(T^{*} M \times M\right)$ is transversal to $N_{\pi}:=\left\{\alpha \in T^{*}\left(T^{*} M \times M\right) \mid \alpha(v)=0\right.$ for all $v \in$ ker $\left.\pi_{*}\right\} \subset T^{*}\left(T^{*} M \times M\right)$, where $\pi: T^{*}\left(T^{*} M \times M\right) \longrightarrow$ $M \times M, \pi\left(\alpha_{x}, y\right)=(x, y)$

and

2. $d \varphi\left(T^{*} M \backslash 0 \times M\right)_{N_{\pi}}:=\left[d \varphi\left(T^{*} M \backslash 0 \times M\right) \cap N_{\pi}\right] / \mathcal{N}_{\pi}^{\perp}=\Gamma(e) \subset\left(T^{*} M \backslash\right.$ $\left.0 \times T^{*} M \backslash 0, \omega \ominus \omega\right)$, where $\mathcal{N}_{\pi}^{\perp}$ is the foliation by isotropic submanifolds generated by the $\omega$-orthogonal bundle $\left(T N_{\pi}\right)^{\perp}$ in $T T^{*}\left(T^{*} M \times M\right)$.

Example. For $M^{n}=\mathbf{R}^{n}$ the function $\varphi: T^{*} \mathbf{R}^{n} \times \mathbf{R}^{n} \longrightarrow \mathbf{R} \varphi\left(\xi_{x}, y\right)=<$ $\xi, x-y>$, is a global phase function for $e$. Consider $0<\delta<r_{i n j}(M, g)$, $\Omega_{\delta}=\left\{\left(\xi_{x}, y\right) \in T^{*} M \times M \mid d(x, y)<\delta\right\} \subset T^{*} M \times M$. In the definition above it is possible to replace $\varphi: T^{*} M \times M \rightarrow \mathbf{R}$ by $\left.\varphi\right|_{\Omega_{\delta}}: \Omega_{\delta} \subset T^{*} M \times M \rightarrow \mathbf{R}$ if $\Omega_{\delta}$ contains the whole fibers of $T^{*} M$.

Lemma 5.10. The function $\varphi_{0}: \Omega_{\delta} \rightarrow \mathbf{R}$ defined by $\varphi_{0}\left(\xi_{x}, y\right)=$ $\xi_{x}\left(\exp _{x}^{-1}(y)\right)$, is a global phase function for $e=i d \in \mathcal{D}_{\theta, 0}^{r+1}$ on $\Omega_{\delta}$.

We refer to [1], p. 541, 42 for the proof which is local in character and does not depend on the compactness or openness of $M$. 
For $\delta<r_{i n j}(M, g)$ we can define global symbols $a(x, \xi) \in \mathcal{U} S^{q}(\Omega)$ on $\Omega=\Omega_{\delta}$ by requiring

$$
\left|\nabla_{\xi}^{\alpha} \nabla_{x}^{\beta} a(x, \xi)\right| \leq C_{\alpha, \beta}\left(1+|\xi|^{q-\alpha}\right.
$$

and assuming an asymptotic expansion of the type (5.10), (5.11).

Let $\chi(x, y)$ be a bump function on $M \times M$ such that supp $\chi \subset$ $\mathcal{U}_{\delta}($ diagonal $)=\mathcal{U}_{\delta}(\triangle)$ and $\chi \equiv 1$ on a neighborhood of the diagonal.

Proposition 5.11. Let $a(x, \xi) \in \mathcal{U} S^{q}(\Omega)$ be a global classical symbol of order $q$. Then

$$
u(x) \longmapsto A u(x):=(2 \pi)^{-n} \int_{T_{x}^{*} M} \int_{B_{\delta}(x)} \chi(x, y) e^{i \varphi_{0}\left(\xi_{x}, y\right)} a(x, \xi)\left|\operatorname{det} \exp _{x *}\right| d y d \xi
$$

is a classical pseudodifferential operator of order $q$ in the former sense, i.e., $A \in \mathcal{U} \Psi^{q}(m)$.

Proof. The fact that $A$ is a $\Psi \mathrm{DO}$ is well known and follows from the famous Kuranishi principle. The uniform boundedness (5.5) of the family $\left\{a_{m}\right\}_{m}$ follows from 5.10 and 5.25 .

Now we return to our first task to define a chart in $\mathcal{D}_{\theta, 0}^{r+1}$. The most simple idea would be to endow $\mathcal{D}_{0}^{r+1} \times \mathcal{F}_{0}^{r+1}$ with a Riemannian metric $G$, to take the induced metric $G_{\theta}$ on the submanifold $\mathcal{D}_{\theta, 0}^{r+1}$ and then apply the Riemannian exponential map of $G$ to a sufficiently small ball in $T_{(i d, 1)} \mathcal{D}_{\theta, 0}^{r+1}$. But this will not work since $\mathcal{D}_{\theta, 0}^{r+1}$ is definitely not a geodesic submanifold, at least for any reasonable metric on $\mathcal{D}_{0}^{r+1} \times \mathcal{F}_{0}^{r+1}$. We could take the Riemannian exponential of $G_{\theta}$, but we don't know it, i.e., we can't calculate or estimate this. Therefore we will construct a chart centered at $i d: T^{*} M \backslash$ $0 \rightarrow T^{*} M \backslash 0$ by another method. The framework of our approach here is already carried out for compact mànifolds in $4.2,4.3,4.4$ of [1], but the case $M^{n}$ open has its own features. It requires additional estimates at $\infty$.

Assume $(W, g)$ satisfies the conditions $(I)$ and $\left(B_{\infty}\right)$. Let $y \in W, \delta<$ $r_{i n j}$ and $\left(U^{\delta}(y), y^{1}, \ldots, y^{n}\right)$ a normal chart. Let $v=v^{1} \frac{\partial}{\partial y^{1}}+\cdots+v^{n} \frac{\partial}{\partial y^{n}}$ be a locally defined vector field, $|v|=\left(v^{i} v_{i}\right)^{\frac{1}{2}}$ its Riemannian norm and $|v|_{f l}=\left(\sum_{i=1}^{n}\left(v^{i}\right)^{2}\right)^{\frac{1}{2}}$ its euclidean flat pointwise norm respectively. We want to compare $|v|$ and $|v|_{f l}$. More generally, consider a locally defined tensor 
field $t=\left(t_{j_{1} \cdots j_{l}}^{i_{1} \cdots i_{k}}\right)$. Denote as before by $|t|$ its Riemannian norm and by $|t|_{f l}=\left(\sum\left(t_{j_{1} \cdots j_{l}}^{i_{1} \cdots i_{k}}\right)^{2}\right)^{\frac{1}{2}}$ its flat euclidean norm. Moreover, denote by $|t|_{U, r+1, f l}$ the $(r+1)$ - Sobolev norm based on Riemannian covariant derivatives, the Riemannian volume element and the flat pointwise norm.

\section{Lemma 5.12.}

a) There exists constants $c_{1}, c_{2}>0$ such that for any $(k, l)$-tensor $t$

$$
c_{1}\left|\nabla^{i} t\right|_{x, f l} \leq\left|\nabla^{i} t\right|_{x} \leq c_{2}\left|\nabla^{i} t\right|_{x, f l}
$$

for all $x \in U_{\delta}, 0 \leq i \leq r+1,, c_{1}, c_{2}$ independent of $y$, depending only on $k, l, r$.

b) If $t \in \Omega^{0,2, r+1}\left(T_{l}^{k} W\right)$ and $\mathcal{U}=\left\{\left(U_{\lambda}, \phi_{\lambda}\right)\right\}_{\lambda}$ is an uniform cover of $W$ by normal charts of radius $\delta$ then there exists constants $d_{1}, d_{2}>0$ such that

$$
d_{1} \sum_{\lambda}|t|_{U_{\lambda}, r+1, f l} \leq|t|_{r+1} \leq d_{2} \sum_{\lambda}|t|_{U_{\lambda}, r+1, f l}
$$

Proof. a) In $U_{\delta}(x)$ holds

$$
\begin{aligned}
& k_{1} \delta_{i j} \xi^{i} \xi^{j} \leq g_{i j} \xi^{i} \xi^{j} \leq k_{2} \delta_{i j} \xi^{i} \xi^{j} \\
& k_{3} \delta^{i j} \xi_{i} \xi_{j} \leq g^{i j} \xi_{i} \xi_{j} \leq k_{4} \delta^{i j} \xi_{i} \xi_{j} .
\end{aligned}
$$

b) This follows from a) using a partition of unity that is bounded up to order $r+1$, the module structure theorem and the fact that the cover is uniformly locally finite.

Now we generalize our notion of a global phase function $\varphi_{H}$ for $e=$ $i d \in \mathcal{D}_{\theta, 0}^{r+1}\left(T^{*} M \backslash 0\right)$ from above to other $f \in \mathcal{D}_{\theta, 0}^{r+1}\left(T^{*} M \backslash 0\right)$. Consider again the projection $\pi: T^{*} M \times M \longrightarrow M, \pi\left(\alpha_{x}, y\right)=(x, y)$, the conormals $N_{\pi} \subset T^{*}\left(T^{*} M \times M\right)$ of this submersion and the corresponding foliation $\mathcal{N}_{\pi}^{\perp}$ of $N_{\pi}$. A function $\varphi: T^{*} M \times M \longrightarrow \mathbf{R}$ is called a global phase function for a diffeomorphism $f$ of $T^{*} M \backslash 0$ iff:

1. $d \varphi\left(T^{*} M \times M\right) \subset T^{*}\left(T^{*} M \times M\right)$ is transversal to $N_{\pi}$, and

2. $\Gamma(f)=d \varphi\left(T^{*} M \times M\right)_{N_{\pi}}:=\left[\left(d \varphi\left(T^{*} M \times M\right) \cap N_{\pi}\right) / \mathcal{N}_{\pi}^{\perp}\right]^{\prime}$, where $C^{\prime}:=$ $\{(x, y, \xi,-\eta) \mid(x, y, \xi \eta) \in C\}$. 
For our purposes we can restrict ourselves to maps $\varphi: \Omega_{\delta} \subset T^{*} M \times$ $M \longrightarrow \mathbf{R}$.

In local charts we have the following representations:

$N_{\pi}=\{(x, \alpha, \vartheta, 0, y, \eta)\}$

$\mathcal{N}_{\pi}^{\perp}=\left\{\left(x, \mathbf{R}^{n}, \vartheta, 0, y, \eta\right)\right\}=$ one leaf

$\left.d \varphi\right|_{\left(\alpha_{x}, y\right)}=\left(x, \alpha, d_{x} \varphi, d_{\alpha} \varphi, y, d_{y} \varphi\right)$,

hence we have locally

$$
\begin{aligned}
d \varphi(\Omega)_{N_{\pi}} & =\left(d \varphi(\Omega) \cap N_{\pi}\right) / \mathcal{N}_{\pi}^{\perp} \\
& =\left\{\left(x, \alpha, d_{x} \varphi, d_{\alpha} \varphi=0, y, d_{y} \varphi\right) \mid(\alpha, y) \in \Omega\right\} \\
& =\left\{\left(x, y, d_{x} \varphi, d_{y} \varphi\right) \mid d_{\alpha} \varphi=0\right\} .
\end{aligned}
$$

We prescribe a $\varphi$ and want to construct the corresponding $f$. For $(x, y) \in U_{\delta}(\Delta) \subset M \times M$ denote $v(x, y)=\exp _{x}^{-1}(y) \in T_{x} M$ and as above $\varphi_{0}\left(\alpha_{x}, y\right)=<\alpha, v(x, y)>$.

Lemma 5.13. Let $h \in \Omega^{0,2, r+2}(S),|h|_{r+2}<\varepsilon, \varepsilon$ sufficiently small and $H$ be the extension homogeneous of degree one to $T^{*} M \backslash 0$. Define $\varphi_{H}: \Omega \subset$ $T^{*} M \backslash 0 \times M \rightarrow \mathbf{R}$ by

$$
\varphi_{H}\left(\alpha_{x}, y\right):=\varphi_{0}\left(\alpha_{x}, y\right)+H\left(\alpha_{x}\right)=\langle\alpha, v(x, y)\rangle+H(x, \alpha)
$$

Then there exists an $f \in \mathcal{D}_{\theta, 0}^{r+1}\left(T^{*} M \backslash 0\right)$ such that $\varphi_{H}$ is a global phase function for $\Gamma(f)$ i.e., $d \varphi_{H}\left(\left(T^{*} M \backslash 0\right) \times M\right)_{N_{\pi}}=\Gamma(f)$.

Proof. Let $y \in M$ and $e_{1}, \ldots, e_{n}$ be an orthonormal base of $T_{y} M$. Let $\delta<$ $r_{i n j}$ and $\left(U^{\delta}(y), y^{1}, \ldots, y^{n}\right)$ the corresponding normal chart. Then $v(x, y) \in$ $T_{x} M$ and $\alpha \in T_{x}^{*} M$ can be written uniquely as $v=\left.v^{1} \frac{\partial}{\partial y^{1}}\right|_{x}+\cdots+\left.v^{n} \frac{\partial}{\partial y^{n}}\right|_{x}$ and $\alpha=\alpha_{1} d y^{1}+\cdots+\alpha_{n} d y^{n}$ respectively. The transversality property is substantially a local property and has been established in [1]. We must show that $\left[d \varphi_{H}(\Omega)_{N_{\pi}}\right]^{\prime}=\Gamma(f)$ for some $f \in \mathcal{D}_{\theta, 0}^{r+1}\left(T^{*} M \backslash 0\right)$, if $|h|_{r+2}<\varepsilon$, for $\varepsilon$ sufficiently small. The Lagrangian submanifold in $T^{*} M \backslash 0 \times T^{*} M \backslash 0$ generated by $\varphi_{H}$ can, according to (5.31), locally be written as

$$
\left\{\left(x, y, d_{x} \varphi_{H}, d_{y} \varphi_{H} \mid d_{\alpha} \varphi_{H}=0\right\}\right.
$$

We assume that $(y, \eta) \in S\left(T^{*} M\right)$ be given and we have to solve for $(x, \alpha)$, i.e we have to solve the equations

$$
d_{y} \varphi_{H}=d_{y}\langle\alpha, v\rangle=\left\langle\alpha, d_{y} v\right\rangle=-\eta
$$




$$
d_{\alpha} \varphi_{H}=0 .
$$

(5.32) yields

$$
\left(\begin{array}{c}
\alpha_{1} \\
\cdot \\
\cdot \\
\cdot \\
\alpha_{n}
\end{array}\right)=-\left(\begin{array}{ccc}
\frac{\partial v^{1}}{\partial y^{1}} & \cdots & \frac{\partial v^{n}}{\partial y^{1}} \\
\cdot & & \cdot \\
\cdot & & \cdot \\
\cdot & & \cdot \\
\frac{\partial v^{1}}{\partial y^{n}} & \cdots & \frac{\partial v^{n}}{\partial y^{n}}
\end{array}\right)_{\mid y}^{-1}\left(\begin{array}{c}
y_{1} \\
\cdot \\
\cdot \\
\cdot \\
y_{n}
\end{array}\right)
$$

where $\eta=\left.\eta_{1} d y^{1}\right|_{y}+\cdots+\left.\eta_{n} d y^{n}\right|_{y}$. The matrix $(\cdots)^{-1}$ equals to $\frac{\partial\left(y^{1}, \ldots, y^{n}\right)}{\partial\left(v^{1}, \ldots, v^{n}\right)}$, i.e., we obtain

$$
\alpha_{i}=-\frac{\partial y^{j}}{\partial v^{i}} \eta^{j}, \quad \alpha=-\left.\frac{\partial y^{j}}{\partial v^{i}} \eta^{j} d y^{i}\right|_{x} .
$$

(5.34) can be reformulated as follows: The map $v:(x, y) \mapsto \exp _{x}^{-1}(y)$ maps $U_{\delta}(\Delta)$ into $T M$. Fixing (the unknown) $x$ for a moment, we obtain a map $\exp _{x}^{-1}():. U_{\delta}(x) \longrightarrow T_{x} M$, its $y$ - differential maps $T_{y} U_{\delta}(x) \longrightarrow T_{v(x, y)} T_{x} M$ and $\left(v(x, .)_{*, y}\right)^{-1}=\left(v(x, .)^{-1}\right)_{*, y}=\left(\exp _{x}\right)_{*, y}: T_{v(x, y)} T_{x} M \longrightarrow T_{y} U_{\delta}$, i.e., we can write

$$
\alpha=-\eta \circ\left(\exp _{x}\right)_{*, y}
$$

The equation (5.33) then becomes

$$
\begin{gathered}
d_{\alpha} \varphi_{H}=d_{\alpha}\langle\alpha, v(x, y)\rangle+d_{\alpha} H=0 \\
v^{1} d \alpha^{1}+\cdots v^{n} d \alpha^{n}=-\left.\left(\frac{\partial H}{\partial \alpha_{1}} d \alpha_{1}+\cdots \frac{\partial H}{\partial \alpha_{n}} d \alpha_{n}\right)\right|_{-\eta v_{*, y}^{-1}},
\end{gathered}
$$

which we have to solve for $x$. Equation (5.36) as an equation between locally defined vector fields is equivalent to $v^{i}=-\left.\frac{\partial H}{\partial \alpha_{i}}\right|_{-\eta v_{*, y}^{-1}}, \quad 1 \leq i \leq n$, which is equivalent to

$$
\left.v^{1} \frac{\partial}{\partial y^{1}}\right|_{x}+\cdots+\left.v^{n} \frac{\partial}{\partial y^{n}}\right|_{x}=-\left.\left(\left.\frac{\partial H}{\partial \alpha_{1}} \frac{\partial}{\partial y^{1}}\right|_{x}+\cdots+\left.\frac{\partial H}{\partial \alpha_{n}} \frac{\partial}{\partial y^{n}}\right|_{x}\right)\right|_{-\eta v_{*, y}^{-1}},
$$

which we have to solve for $x$. There are several way to solve (5.37). Write the right hand side of (5.37) as $-\left\langle\frac{\partial H}{\partial \alpha}, \frac{\partial}{\partial y} \mid x\right\rangle$, we can assume $c_{2}\left|\left\langle\frac{\partial H}{\partial \alpha}, \frac{\partial}{\partial y}\right\rangle\right|_{f l}=\delta_{1}<\delta$, (cf.(5.27)). Consider the map $F:[0,1] \times$ 
$U_{\delta}(y) \longrightarrow V_{\delta}\left(0_{T U_{\delta}}\right), F(t, x)=\exp _{x}^{-1}(y)+t\left\langle\frac{\partial H}{\partial \alpha},\left.\frac{\partial}{\partial y}\right|_{x}\right\rangle$ and set $L:=\{t \in$ $[0,1] \mid$ there exists a unique $x_{t}$ such that $\left.F\left(t, x_{t}\right)=0\right\}$. Then the following hold:

1) $L \neq \emptyset$, since $0 \in L$ with $x_{0}=y$.

2) There exists a $\lambda>0$ such that $[0, \lambda[\subset L$. This follows from the fact that $\left.d_{x} F\right|_{(0, y)}$ is invertible.

3) $L$ is open, which follows analogous to 2 ).

4) $L$ is closed: assume $t_{1}<t_{2}<\cdots \rightarrow t^{*}$, this implies $x_{t_{\nu}} \in U_{t^{*} \delta}$, there exists a convergent subsequence $x_{t_{\nu_{i}}} \rightarrow x^{*}$ and $F\left(t^{*}, x^{*}\right)=0$. Therefore $L=[0,1]$ and there exists a unique $x \in U_{\delta}(y)$ such that $\exp _{x}^{-1}(y)=$ $-\left.\left\langle\frac{\partial H}{\partial \alpha},\left.\frac{\partial}{\partial y}\right|_{x}\right\rangle\right|_{\alpha=-\eta v_{*, y}^{-1}}$.

What remains to assure is that $c_{2}\left|\left\langle\frac{\partial H}{\partial \alpha}, \frac{\partial}{\partial y}\right\rangle\right|_{f l}<\delta$. This can been seen as follows: For a covector $V \in T^{*} T^{*} M$ we have $|V|=g(K V, K V)+g\left(V_{h}, V_{h}\right)$ where $K: T^{*} T^{*} M \longrightarrow T_{v e r t}^{*} T^{*} M$ is the connection projection. Any $V \in$ $T^{*} T^{*} U_{\delta}$ can be written as $V=A_{1} d y^{1}+\cdots+A_{n} d y^{n}+B_{1} d \alpha^{1}+\cdots+B_{n} d \alpha^{n}$. If $V=B_{1} d y^{1}+\cdots+B_{n} d y^{n}$, then it is purely vertical and $g_{\text {Sasaki }}(V, V)=$ $g(K V, K V)=g(V, V)$. This can be calculated from the $g^{i j}(x)$ and the $B_{i}^{\prime} s$ i.e., we can apply (5.27). Using uniform boundedness, properties of $\exp$ (cf. [15]) we derive from (5.27) and (5.34), (5.35)

$$
\frac{1}{2}=\frac{1}{2}|\eta|_{y} \leq|\alpha|_{x} \leq \frac{3}{2}|\eta|_{y}=\frac{3}{2}
$$

if we choose $\delta<r_{i n j}$ small enough. If we assume this has been done, $\alpha$ lies in a $\frac{1}{2}$ - neighborhood of $S\left(T^{*} M\right)$. By assumption $\left.H\right|_{S}=h \in \Omega^{0,2, r+2}(S)$,

$$
\begin{gathered}
{ }^{b}|h| \leq C_{0}|h|_{r+2} \\
{ }^{b}\left|d_{\alpha} H\right|_{U_{\frac{1}{2}}(S)} \leq{ }^{b}|d H|_{U_{\frac{1}{2}}(S)} \leq \frac{1}{2}{ }^{b}\left|d_{S} h\right|+{ }^{b}|h| \\
\leq \frac{1}{2} C_{1}\left|d_{S} h\right|_{r+1}+C_{0}|h|_{r+2} \leq C_{2}|h|_{r+2},
\end{gathered}
$$

hence$$
\leq c_{2} c_{1}{ }^{b}\left|d_{\alpha} H\right|_{U_{\frac{1}{2}}(S)} \leq c_{2} c_{1}{ }^{b}|d H|_{U_{\frac{1}{2}}(S)} \leq c_{2} c_{1} C_{2}|h|_{r+2}=C_{3}|h|_{r+2} .
$$

Choosing $|h|_{r+2} \leq \frac{\delta}{C_{3}}$ we obtain a unique $x$ solving equation (5.33). We get an orthonormal base at $x$ by parallel translation of $e_{1}, \ldots, e_{n} \in T_{y} U_{\delta}$ 
from $y$ to $x$ along the unique connecting geodesic. This yields a normal chard $\left(U_{\delta}(x), x^{1}, \ldots, x^{n}\right), y \in U_{\delta}(x)$. We now insert $x$ into $\left\langle\alpha, d_{x} v(x, y)\right\rangle+$ $d_{x} H(x, \alpha)$ and obtain

$$
\begin{aligned}
\xi(x, y) & =\left\langle\alpha, d_{x} v(x, y)\right\rangle+d_{x} H(x, \alpha) \\
& =-\left(\frac{\partial y^{j}}{\partial v^{1}} \eta^{j} d_{x} v^{1}+\cdots+\frac{\partial y^{j}}{\partial v^{n}} \eta^{j} d_{x} v^{n}\right)+d_{x} H \\
& =-\left.\left.\frac{\partial v^{k}}{\partial x^{l}}\right|_{x} \frac{\partial y^{j}}{\partial v^{k}}\right|_{v^{-1}(y)} \eta_{j} d x^{l}+\left.\frac{\partial H}{\partial x^{l}}\right|_{x} d x^{l} .
\end{aligned}
$$

The coordinate free description of $\left\langle\alpha, d_{x} v(x, y)\right\rangle$ is given by

$$
\begin{aligned}
\left\langle\alpha, d_{x} v(x, y)\right\rangle= & -\beta \circ\left[\left(\exp _{x}\right)_{* y}: T_{v(x, y)} T_{x} M \rightarrow T_{y} U_{\delta}(y)\right] \circ \\
\circ & {\left[\left(\exp _{(.)}^{-1}(y)_{* y}: T_{x} U_{\delta}(x) \rightarrow T_{v(x, y)} T_{x} M\right] .\right.}
\end{aligned}
$$

If $0 \neq \eta \notin S\left(T^{*} M\right)$, then $\alpha=-\eta \circ v_{*, y}^{-1}$ remains, but to determine $x$ we apply the procedure above with $\eta^{\prime}=\eta /|\eta|$ and $\alpha^{\prime}=\eta^{\prime} \circ v_{*, y}^{-1}$ whereas $\xi$ is again defined by (5.41). Thus we finally get a map $f^{-1}(y, \eta)=(x(y, \eta), \xi(y, \eta))$. This map is well defined. The determining equations and its solutions can be described coordinate free as shown by (5.35) and (5.42). Using the geodesic convexity of normal charts and the implicit function theorem, it is easy to derive that $f^{-1}$ is $1-1$, onto and of class $C^{1}$ together with its inverse. $f$ and $f^{-1}$ are $C^{1}$ - diffeomorphisms of $T^{*} M \backslash 0$. The fact that $d \varphi_{H}\left(T^{*} M \backslash 0 \times M\right)$ is a conic Lagrangian submanifold implies that $f^{*} \theta=\theta$, hence $f \in C^{1} \mathcal{D}_{\theta}\left(T^{*} M \backslash 0\right)$. To assure that $f \in \mathcal{D}_{\theta, 0}^{r+1}(T * M \backslash 0)$ we have to show that the map $f^{-1}: S\left(T^{*} M\right) \ni \eta_{y} \longrightarrow \xi_{x} /\left|\xi_{x}\right| \in S\left(T^{*} M\right)$ belongs to $\mathcal{D}_{\theta, 0}^{r+1}\left(S\left(T^{*} M\right)\right)$. To assure that this new $f^{-1} \in \mathcal{D}_{\theta}^{r+1}\left(S\left(T^{*} M\right)\right)$ it would be sufficient that dist $\left(\left(x, \xi_{x} /\left|\xi_{x}\right|\right),(y, \eta)\right)<r_{i n j} S\left(T^{*} M\right)$ and that the corresponding vector field on $S\left(T^{*} M\right)$ would be of class $r+1$. Hence we have to estimate form above dist $(\xi /|\xi|, \eta)$. For this we need an arc from $\xi$ to $\eta$. First consider the parallel translation of $\xi /|\xi|$ to the fiber $S\left(T_{y}^{*} M\right)$ along the geodesic $\exp (s v(x, y)), 0 \leq s \leq 1, s \mapsto P_{s} \xi /|\xi|, \quad P_{1} \xi /|\xi| \in S\left(T_{y}^{*} M\right)$. This is a horizontal geodesic in $S\left(T^{*} M\right)$ covering the geodesic $\exp (s v)$. We obtain length $\left\{P_{s} \xi /|\xi|\right\}=\operatorname{length}\{\exp (s v)\}=|v|$, dist $\left(\xi, P_{1} \xi /|\xi|\right)=\operatorname{dist}(x, y)=$ $|v|$. But $P_{1} \xi /|\xi|$ and $\eta$ lie in the fiber $S\left(T_{y}^{*} M\right)$ which is an euclidean sphere. The distance of two points is the length of the shortest geodesic between them. But this distance can be estimated from above by the distance in $T_{y}^{*} M$ multiplied by a factor $C_{4} \approx 1.8$, i.e., $\operatorname{dist}\left(P_{1} \xi /|\xi|, \eta\right) \leq C_{4}\left|P_{1} \xi /\right| \xi|-\eta|$. 
Now

$$
\begin{array}{r}
\quad|P \xi /| \xi|-\eta|=\left|\frac{P \xi|\eta|-\eta|\xi|}{|\xi|}\right|=\left|\frac{P \xi|\eta|-P \xi|\xi|+P \xi|\xi|-\eta|\xi|}{|\xi|}\right| \\
\leq\left|\frac{P \xi(|\eta|-|\xi|)}{\xi \mid}\right|+|P \xi-\eta|=|| \eta|-| \xi||+|P \xi-\eta| \leq 2|P \xi-\eta| .
\end{array}
$$

If $\xi=\left.\xi_{i} d x^{i}\right|_{x}$ and $P \xi=\left.\left(\xi_{i}+\Delta \xi_{i}\right) d x^{i}\right|_{y}$ then $\xi_{i}+\Delta \xi_{i}$ is the solution $\xi_{i}(|v|)$ of the equation

$$
\frac{d \xi_{i}(t)}{d t}-\Gamma_{i j}^{k} \xi_{k}(t) \frac{d x^{j}(t)}{d t}=0
$$

where $x^{j}(t)$ are the coordinates of the geodesic $\exp \left(\frac{t}{|v|} v\right), \xi_{i}(0)=\xi_{i}$. It is a simple and well known fact that $|\Delta \xi|$ can be estimated as

$$
|\Delta \xi| \leq C_{5} \cdot \Gamma \cdot|v| \cdot|\xi|=C_{6} \operatorname{dist}(x, y) \cdot|\xi|
$$

Hence

$$
|P \xi-\eta|_{y}=\left|\xi_{i} d x_{\mid y}^{i}+\Delta \xi_{i} d x_{\mid y}^{i}-\eta\right| \leq\left|\xi_{i} d x_{\mid y}^{i}-\eta\right|+C_{6} \operatorname{dist}(x, y) \cdot|\xi| .
$$

We insert $\xi_{l} d x^{l}$ from (5.41) and $\eta=\left.\eta_{i} d y^{i}\right|_{y}=\left.\frac{\partial y^{j}}{\partial x^{l}} \eta_{j} d x^{l}\right|_{y}$ and obtain

$$
\begin{aligned}
\left|\xi_{l} d x_{\mid y}^{l}-\eta\right| & \leq\left.\left|-\frac{\partial v^{k}}{\partial x^{l}}\right|_{x} \frac{\partial y^{j}}{\partial v^{k}}\right|_{v^{-1}(y)} \eta_{j}-\left.\left.\frac{\partial y^{j}}{\partial x^{l}}\right|_{y} \eta_{j} d x^{l}\right|_{y}+\left.\left.\left|\frac{\partial H}{\partial x^{l}}\right|_{x} d x^{l}\right|_{y}\right|_{y} \\
5.44) & \leq\left.\left|-\frac{\partial v^{k}}{\partial x^{l}}\right|_{x} \frac{\partial y^{j}}{\partial v^{k}}\right|_{v^{-1}(y)} \eta_{j}-\left.\left.\frac{\partial y^{j}}{\partial x^{l}}\right|_{y} \eta_{j} d x^{l}\right|_{f l}+\left.\left.\left|\frac{\partial H}{\partial x^{l}}\right|_{x} d x^{l}\right|_{y}\right|_{y}
\end{aligned}
$$

Next we use the following uniform expansions on $M$ :

$$
\begin{aligned}
& \left.\frac{\partial\left(y^{1}, \ldots, y^{n}\right)}{\partial\left(v^{1}, \ldots, v^{n}\right)}\right|_{v^{-1}(y)}=\left(\begin{array}{lll}
1 & & 0 \\
& \ddots & \\
0 & & 1
\end{array}\right)+O(\operatorname{dist}(x, y))+o(\operatorname{dist}(x, y)), \\
& \left.\frac{\partial\left(v^{1}, \ldots, v^{n}\right)}{\partial\left(x^{1}, \ldots, x^{n}\right)}\right|_{x}=-\left(\begin{array}{ccc}
1 & & 0 \\
& \ddots & \\
0 & & 1
\end{array}\right)+O(\operatorname{dist}(x, y))+o(\operatorname{dist}(x, y)), \\
& \left.\frac{\partial\left(y^{1}, \ldots, y^{n}\right)}{\partial\left(x^{1}, \ldots, x^{n}\right)}\right|_{x(y)}=\left(\begin{array}{lll}
1 & & 0 \\
& \ddots & \\
0 & & 1
\end{array}\right)+O(\operatorname{dist}(x, y))+o(\operatorname{dist}(x, y)) .
\end{aligned}
$$


Taking this into account and $|\eta|_{y}=1$ we can conclude that the right hand side of (5.44) can be estimated as

$$
\begin{aligned}
(5.44) & \leq C_{7}|\eta| \operatorname{dist}(x, y)+\frac{3}{2}\left|d_{x} H(x, \alpha /|\alpha|)\right| \\
& \leq C_{7} \operatorname{dist}(x, y)+\frac{3}{2}{ }^{b}\left|d_{S} h\right| \leq C_{7} \operatorname{dist}(x, y)+C_{8}|h|_{r+2} .
\end{aligned}
$$

There remains to estimate $|\xi|$ itself .

$$
\begin{aligned}
|\xi|_{x} & \leq\left|\left\langle\alpha, d_{x} v\right\rangle\right|_{x}+\left|d_{x} H(x, \alpha)\right|_{x} \leq c_{2}\left|\left\langle\alpha, d_{x} v\right\rangle\right|_{f l}+C_{8}|h|_{r+2} \\
& \leq c_{2}\left(|\eta|+C_{9}|\eta| \operatorname{dist}(x, y)\right)+C_{8}|h|_{r+2}=c_{2}+C_{10} \operatorname{dist}(x, y)+C_{8}|h|_{r+2} .
\end{aligned}
$$

We now reached our final estimate:

$$
\begin{aligned}
& \operatorname{dist}(\xi, \eta) \\
& \leq \operatorname{dist}(x, y)+2 C_{4}\left[C_{6}|\xi| \operatorname{dist}(x, y)+C_{7} \operatorname{dist}(x, y)+C_{8}|h|_{r+2}\right] \\
& \leq \operatorname{dist}(x, y)+2 C_{4}\left[C_{6}\left(c_{2}+C_{10} \operatorname{dist}(x, y)+C_{8}|h|_{r+2}\right) \operatorname{dist}(x, y)+\right. \\
&\left.\quad+C_{7} \operatorname{dist}(x, y)+C_{8}|h|_{r+2}\right] \\
& \leq C_{3}|h|_{r+2}+2 C_{4}\left[C_{6}\left(c_{2}+C_{10} C_{3}|h|_{r+2}+C_{8}|h|_{r+2}\right) C_{3}|h|_{r+2}+\right. \\
&\left.+C_{7} C_{3}|h|_{r+2}+C_{8}|h|_{r+2}\right] \\
& \leq C_{11}|h|_{r+2}+C_{12}|h|_{r+2}^{2} \leq C_{13}|h|_{r+2}, \quad \text { if }|h|_{r+2} \leq 1 .
\end{aligned}
$$

We conclude, that if

$$
\varepsilon_{1}=\min \left\{1, \frac{\delta}{C_{3}}, \frac{r_{i n j}(S)}{C_{13}}\right\}, \text { and }|h|_{r+2}<\varepsilon_{1}
$$

then there exists a unique vector field $X(h)$ such that

$$
f=\exp X(h) .
$$

To obtain $f \in \mathcal{D}_{0}^{r+1}(S)$ we must in addition show that $|X(h)|_{r+1}<\infty$. This is a rather long and technical estimate, but the established inequalities carry over in a quite natural manner step by step to pointwise norms of derivatives (here we use repeatedly (5.27)) and finally to Sobolev norms, applying the module structure theorem. We omit the details here. We established that $f \in C^{1} \mathcal{D}_{\theta}\left(T^{*} M \backslash 0\right)$ and $\left.f\right|_{S}=\exp X(h) \in C^{1} \mathcal{D}_{0}(S)$.

If we define $\beta$ by (4.6) we obtain $\left(\left.f\right|_{S}=\exp X(h), \beta\right) \in \mathcal{D}_{\theta, 0}^{r+1}(S)$, i.e., the extended $f \in \mathcal{D}_{\theta, 0}^{r+1}\left(T^{*} M \backslash 0\right)$.

Now we sharpen our considerations by proving 
Lemma 5.14. The map $h \longmapsto H \longmapsto f$ given by Lemma 5.13 is a bijection from a neighborhood $\mathcal{V}=\mathcal{V}_{\varepsilon}(0) \subset \Omega^{0,2, r+2}(S)$ onto a neighborhood $\mathcal{U}=$ $\mathcal{U}(i d) \subset \mathcal{D}_{\theta, 0}^{r+1}\left(T^{*} M \backslash 0\right)$ of $i d \in \mathcal{D}_{\theta, 0}^{r+1}\left(T^{*} M \backslash 0\right)$. The inverse mapping is given by

$$
f \longmapsto H=H_{f}, \quad H\left(\alpha_{x}\right)=-\left\langle\alpha_{x}, \exp _{x}^{-1}\left(\pi\left(f^{-1}\left(\alpha_{x}\right)\right)\right)\right\rangle .
$$

Proof. First we recall the local topology of $\mathcal{D}_{\theta, 0}^{r+1}(S)$, giving a neighborhood basis for $i d \in \mathcal{D}_{\theta, 0}^{r+1}(S)$. As established in [10], a neighborhood basis for $i d \in \mathcal{D}_{0}^{r+1}(S)$ is given by $\mathcal{U}=\left\{\mathcal{U}_{\frac{1}{\nu}}(i d)\right\}_{\frac{1}{\nu} \leq r_{i n j}(S)}$, where

$$
\mathcal{U}_{\frac{1}{\nu}}(i d)=\left\{\left.\exp X|| X\right|_{r+1}<\frac{1}{\nu}\right\},(\exp X)(x)=\exp _{x} X(x) .
$$

Then the corresponding basis in $\mathcal{D}_{\theta, 0}^{r+1}(S)$ is given by $\mathcal{U}_{\frac{1}{\nu}, \theta}(i d)=$ $\left\{\mathcal{U}_{\frac{1}{\nu}, \theta}(i d)\right\}_{\frac{1}{\nu} \leq r_{i n j}(S)}$, where

$$
\mathcal{U}_{\frac{1}{\nu}, \theta}(i d)=\mathcal{U}_{\frac{1}{\nu}}(i d) \cap \mathcal{D}_{\theta, 0}^{r+1}(S)
$$

Remark. The $X s$ in (5.49) must not necessarily satisfy $L_{X} \theta=0$ or $L_{X} \theta=u \cdot \theta$. We follow in (5.48), (5.49) the Riemannian exponential, not the integral curves of $X$ with $L_{X} \theta=u \cdot \theta$.

Considering (5.48), we see that $H$ is only well defined if $\exp _{z}^{-1}\left(\pi f^{-1}\left(\xi_{z}\right)\right)$ is well defined, i.e., if

$$
\operatorname{dist}\left(z, \pi f^{-1}\left(\xi_{z}\right)\right)<r_{i n j}(M) .
$$

Clearly, $r_{i n j}\left(S, g_{S}\right) \leq r_{i n j}(M, g)$, since horizontal geodesics project isometrically to geodesics.

For $\varepsilon_{2}$ sufficiently small and $|X|_{r+1}<\varepsilon_{2}$, we have

$$
\operatorname{dist}\left(\xi_{z},(\exp X)^{-1}\left(\xi_{z}\right)\right)<r_{i n j}(S) \leq r_{i n j}(M),
$$

i.e., for $f \in \mathcal{U}_{\varepsilon, \theta}(i d)$, (5.51) is satisfied and

$$
H\left(\xi_{z}\right)=-\left\langle\xi_{z}, \exp _{z}^{-1}\left(\pi f^{-1}\left(\xi_{z}\right)\right)\right\rangle
$$

is well defined. Moreover, the right hand side is homogeneous of degree one. We must still assure that $\left.H\right|_{S} \in \Omega^{0,2, r+2}(S)$. The derivatives of $\left.H\right|_{S}$ lead 
to the derivatives of certain Jacobi fields. Their pointwise norms can be estimated by polynomials on the pointwise norms of the derivatives of $X$ which are additionally square integrable. This has been performed in [10]. At the end we get

$$
\left.H\right|_{S} \in \Omega^{0,2, r+2}(S)
$$

The gain of one Sobolev index comes from the fact $\exp X$ contains already one integration. Moreover given any $\varepsilon_{3}>0$, there exists $\varepsilon_{4}>0$ such that

$$
|X|_{r+1}<\varepsilon_{4} \text { implies }\left.|H|_{S}\right|_{r+2}<\varepsilon_{3} .
$$

The key for proving (5.54) are the Jacobi field constructions, their estimates and the module structure theorem.

Setting $\varepsilon_{3}=\varepsilon_{1}$ from (5.46) and choosing $\varepsilon_{5}=\min \left\{\varepsilon_{2}, \varepsilon_{4}\right\}$, we obtain

$$
\begin{aligned}
& H=H_{f} \text { is for } f \in \mathcal{U}_{\varepsilon_{5}, \theta}(i d) \text { well defined, } \\
& \qquad\left.H\right|_{S} \in \Omega^{0,2, r+2}(S) \text { and }\left.|H|_{S}\right|_{r+2} \leq \varepsilon_{1} .
\end{aligned}
$$

(5.55) now permits to construct the sequence of maps

$$
f \longmapsto H=H_{f} \longmapsto \varphi_{H_{f}} \longmapsto f_{\varphi_{H_{f}}}
$$

In [1] it is proved that (5.56) equals to $i d$. If we set $\varepsilon=\min \left\{\varepsilon_{1}, \varepsilon_{5}\right\}$, the sequence

$$
H \longmapsto \varphi_{H} \longmapsto f=f_{\varphi_{H}} \longmapsto H_{f}
$$

is also well defined and equals to $i d$ according to [1]. We omit the proof that the constructed 1-1 mapping $\Psi: f \longmapsto H_{f} \longmapsto h_{f}=\left.H_{f}\right|_{S}$ is of class $C^{k-r}$.

We summarize our result in

Theorem 5.15. $\left(\mathcal{U}=\mathcal{U}_{\varepsilon, \theta}(i d), \Psi, \Omega^{0,2, r+2}(S)\right)$ is a $C^{k-r}$ chart at id $\in$ $\mathcal{D}_{\theta, 0}^{r+1}\left(T^{*} M \backslash 0\right)$, where

$$
\Psi(f)\left(\alpha_{x}\right)=-\left\langle\alpha_{x}, \exp _{x}^{-1}\left(\pi\left(f^{-1}\left(\alpha_{x}\right)\right)\right)\right\rangle
$$

for all $\alpha_{x} \in T^{*} M \backslash 0$.

Now we are in a position to construct a local section $\sigma: \mathcal{U} \subset \mathcal{D}_{\theta, 0}^{\infty}=$ $\lim _{\leftarrow} \mathcal{D}_{\theta, 0}^{r+1} \longrightarrow\left(\mathcal{U} F^{o, k}(\infty)\right)$. We assume the condition $\left(B_{\infty}\right)$. For $f \in \mathcal{U} \cap \mathcal{D}_{\theta, 0}^{\infty}$ 
and $A \in \mathcal{U} F^{o, k}(f)$ and $a(x, \xi)$ a representative of the classical symbol of $A$ we now can write in analogy to $(5.26)$

$$
A u(x)=(2 \pi)^{-n} \int_{T_{x}^{*} M} \int_{B_{\delta}(x)} \chi(x, y) e^{i \varphi_{H}\left(\alpha_{x}, x\right)} u(y)\left|\operatorname{det} \exp _{*}\right| d y d \xi,
$$

where $\chi$ is a bump function as in (5.26) and $\varphi_{H}$ is the global phase function of $\Gamma(f)$ defined in Lemmas 5.13, 5.14. The formula (5.59) holds modulo $\mathcal{U} \Psi^{-\infty}(M)$.

We define the local section $\sigma$ as follows: Let $f \in \mathcal{U} \cap \mathcal{D}_{\theta, 0}^{\infty}\left(T^{*} M \backslash 0\right)$ and define $\sigma(f) \in\left(\mathcal{U} F^{0, k}(\infty)\right)_{*}$ by

$$
\sigma(f) u(x):=(2 \pi)^{-n} \int_{T_{x}^{*} M} \int_{B_{\delta}(x)} \chi(x, y) e^{i \varphi_{H}\left(\alpha_{x}, y\right)} u(y)\left|\operatorname{det} \exp _{*}\right| d y d \xi
$$

where $\varphi_{H}\left(\alpha_{x}, y\right)=\varphi_{0}\left(\alpha_{x}, y\right)+H\left(\alpha_{x}\right), H=\Psi(f)$, i. e. $\varphi_{H}\left(\alpha_{x}, y\right)=$ $\left\langle\alpha_{x}, \exp _{x}^{-1}(y)\right\rangle-\left\langle\alpha_{x}, \exp _{x}^{-1}\left(\pi\left(f^{-1}\left(\alpha_{x}\right)\right)\right)\right\rangle$.

The operator $\sigma(f)$ is a FIO with smooth phase function $\varphi_{\Psi(f)}$ and amplitude $a=1$. Moreover, $\sigma(f)$ is invertible modulo smoothing operators since $f$ is invertible and its principal symbol is $a=1$, hence $\sigma(f) \in\left(\mathcal{U} F^{0, k}(\infty)\right)_{*}$ for any $k$. Furthermore, $\pi \sigma(f)=f$, hence $\sigma$ is a local section of the exact sequence (5.24).

\section{6. $\left(\mathcal{U} \Psi^{0, k}\right)_{*}$ as ILH Lie group.}

We want to endow $\left(\mathcal{U} \Psi^{0, k}\right)_{*}$ with the structure of an ILH Lie group. Consider $[A] \in \mathcal{U} \Psi^{q}$ (which means $\mathcal{U} \Psi^{q} / \mathcal{U} \Psi^{-\infty}$ ) with principal symbol $a_{q}$ which is globally defined and behaves well under transformations. Let $A_{q}$ be the operator given by (5.26) with total symbol $a_{q}$. Then $\left[A-A_{q}\right] \in \mathcal{U} \Psi^{q-1}$. Let $a_{q-1}$ be the principal symbol of $A-A_{q}$ and $A_{q-1}$ given by (5.26). Then $[A-$ $\left.A_{q}-A_{q-1}\right] \in \mathcal{U} \Psi^{q-2}$. Continuing in the manner, we obtain an assignment

$$
[A] \in \mathcal{U} \Psi^{q} \longmapsto\left(a_{q}(x, \xi), a_{q-1}(x, \xi), \ldots\right)
$$

where $a_{q-1} \in C^{\infty}\left(T^{*} M \backslash 0\right)$, satisfies (5.25) and is homogeneous of degree $q-1$ in $\xi$, hence not square integrable. We consider their restriction to $S\left(T^{*} M\right)$ and denote $\left.a_{q-j}\right|_{S}$ again by $a_{q-j}$. The map (6.1) is still a vector space isomorphism. Fix some $k$ and consider the assignment

$$
[A] \in \mathcal{U} \Psi^{q, k} \longmapsto\left(a_{q}, a_{q-1}, \ldots, a_{-k}\right)
$$


We introduce a uniform Sobolev topology on $\mathcal{U} \Psi^{q, k}$. Let $\delta>0, s>n$ and set

$$
\begin{aligned}
V_{\delta} & =\left\{\left([A],\left[A^{\prime}\right]\right) \in \mathcal{U} \Psi^{q, k} \times \mathcal{U} \Psi^{q, k}||[A]-\left.\left[A^{\prime}\right]\right|_{q, k, s} ^{2}:=\right. \\
& \left.:=\left|a_{q}-a_{q}^{\prime}\right|_{q+k+s}^{2}+\left|a_{q-1}-a_{q-1}^{\prime}\right|_{q+k+s-1}^{2}+\cdots+\left|a_{-k}-a_{-k}^{\prime}\right|_{s}^{2}<\delta^{2}\right\}
\end{aligned}
$$

Lemma 6.1. $L=\left\{V_{\delta}\right\}$ is a basis for a metrizable uniform structure $\mathcal{A}^{q, k, s}$.

We omit the very simple proof.

Let $\mathcal{U} \Psi^{q, k, s}=\overline{\mathcal{U} \Psi^{q, k}} \|_{q, k, s}$ be the completion.

Proposition 6.2. $\mathcal{U} \Psi^{q, k, s}$ is the topological sum of its arc components,

$$
\mathcal{U} \Psi^{q, k, s}=\sum_{i \in I} \operatorname{comp}\left(\left[A_{i}\right]\right)
$$

and each component is a smooth Hilbert manifold. Here

$$
\operatorname{comp}([A])=\left\{\left[A^{\prime}\right] \in \mathcal{U} \Psi^{q, k, s}||[A]-\left[A^{\prime}\right]_{q, k, s} \mid<\infty\right\} .
$$

We omit the simple proof which is performed for spaces of connections or spaces of metrics e.g., in [8], [11].

In $\mathcal{U} \Psi^{0, k}$ composition is well defined.

Proposition 6.3. Composition in $\mathcal{U} \Psi^{0, k}$ extends continuously to $\mathcal{U} \Psi^{0, k, s}$. More precisely, composition is a continuous map

$$
\operatorname{comp}([A]) \times \operatorname{comp}([B])=\operatorname{comp}([A \circ B]) .
$$

Proof. The elements of $\mathcal{U} \Psi^{0, k}$ are dense in $\mathcal{U} \Psi^{0, k, s}$. Fix $[A],[B],[A \circ B] \in$ $\mathcal{U} \Psi^{0, k}$. We have to show that for $\left[A^{\prime}\right] \in \operatorname{comp}([A]),\left[B^{\prime}\right] \in \operatorname{comp}([B])$

$$
\left[A^{\prime} \circ B^{\prime}\right] \in \operatorname{comp}([A \circ B])
$$

and that this map is continuous. The proof of (6.6) will also include the proof of continuity. Represent the operators $[A],[B],[A \circ B]=[C],\left[A^{\prime}\right],\left[B^{\prime}\right],\left[A^{\prime} \circ\right.$ $\left.B^{\prime}\right]=\left[C^{\prime}\right]$ by the symbols 


$$
\begin{aligned}
& a=a(x, \xi)=a_{0}+a_{-1}+\cdots+a_{-k}, \\
& b=b(x, \xi)=b_{0}+b_{-1}+\cdots+b_{-k}, \\
& c=c(x, \xi)=c_{0}+c_{-1}+\cdots+c_{-k}, \\
& a^{\prime}=a^{\prime}(x, \xi)=a_{0}^{\prime}+a_{-1}^{\prime}+\cdots+a_{-k}^{\prime}, \text { etc.. }
\end{aligned}
$$

Then

$$
\begin{gathered}
c_{0}=a_{0} b_{0}, \quad c_{0}^{\prime}=a_{0}^{\prime} b_{0}^{\prime} \\
c_{-1}=a_{0} b_{-1}+a_{-1} b_{0}+\sum_{i=1}^{n} \partial_{x_{i}} a_{0} \partial_{\xi_{i}} b_{0}, \\
c_{-1}^{\prime}=a_{0}^{\prime} b_{-1}^{\prime}+a_{-1}^{\prime} b_{0}^{\prime}+\sum_{i=1}^{n} \partial_{x_{i}} a_{0}^{\prime} \partial_{\xi_{i}} b_{0}^{\prime}, \text { etc. }
\end{gathered}
$$

Then

$$
\begin{aligned}
& \left|a_{0} b_{0}-a_{0}^{\prime} b_{0}^{\prime}\right|_{k+s} \\
& =\left|a_{0} b_{0}-a_{0}^{\prime} b_{0}+a_{0}^{\prime} b_{0}-a_{0}^{\prime} b_{0}^{\prime}\right|_{k+s} \\
& \leq\left|\left(a_{0}-a_{0}^{\prime}\right) b_{0}\right|_{k+s}+\left|a_{0}^{\prime}\left(b_{0}-b_{0}^{\prime}\right)\right|_{k+s} \\
& \leq\left|\left(a_{0}-a_{0}^{\prime}\right) b_{0}\right|_{k+s}+\left|\left(a_{0}^{\prime}-a_{0}\right)\left(b_{0}-b_{0}^{\prime}\right)\right|_{k+s}+\left|a_{0}\left(b_{0}-b_{0}^{\prime}\right)\right|_{k+s} \\
& \leq\left|a_{0}-a_{0}^{\prime}\right|_{k+s}{ }^{b, k+s}\left|b_{0}\right|+C_{0}\left|a_{0}^{\prime}-a_{0}\right|_{k+s}\left|b_{0}-b_{0}^{\prime}\right|_{k+s}+{ }^{b, k+s}\left|a_{0}\right| \cdot\left|b_{0}-b_{0}^{\prime}\right|_{k+s} .
\end{aligned}
$$

Here we applied the module structure theorem in the middle term and in the boundary terms $(5.25)$ for $a_{0}, b_{0}$. Next we have to estimate

$$
\begin{aligned}
\left|c_{-1}-c_{-1}^{\prime}\right|_{k-1+s} \leq & \left|a_{0} b_{-1}-a_{0}^{\prime} b_{-1}^{\prime}\right|_{k-1+s}+\left|a_{-1} b_{0}-a_{-1}^{\prime} b_{0}^{\prime}\right|_{k-1+s} \\
& +\sum_{i=1}^{n}\left|\partial_{x_{i}} a_{0} \partial_{\xi_{i}} b_{0}-\partial_{x_{i}} a_{0}^{\prime} \partial_{\xi_{i}} b_{0}^{\prime}\right|_{k-1+s}
\end{aligned}
$$

Each single term in (6.8) can be estimated as in (6.7). The general case can be proved by a simple but very extensive induction. The key inequality is (6.7) (applied with other indices).

Proposition 6.4. $\left(\mathcal{U} \Psi^{0, k, s}\right)_{*}$ is a Hilbert Lie group.

Proof. According to (6.4), each component is an affine Hilbert space. Proposition 6.3 implies that $\mathcal{U} \Psi^{0, k, s}$ is an affine Hilbert algebra. Then $\left(\mathcal{U} \Psi^{0, k, s}\right)_{*}$ is open and $A \mapsto A^{-1}$ is a homoemorphism. Composition in $\mathcal{U} \Psi^{0, k, s}$ is 
even smooth since it is bilinear and continuous. Hence the same holds in $\left(\mathcal{U} \Psi^{0, k, s}\right)_{*}$.

\section{Remarks.}

1. If the underlying manifold $M$ is compact then $\left(\mathcal{U} \Psi^{0, k, s}\right)_{*}$ consists of one component (as $\mathcal{U} \Psi^{0, k, s}$ does). On open manifolds, $\left(\mathcal{U} \Psi^{0, k, s}\right)_{*}$ consists of uncountably many components (as $\mathcal{U} \Psi^{0, k, s}$ does).

2. If we look in $\left(\mathcal{U} \Psi^{0, k, S}\right)_{*}$ for those $A s$ which are invertible in their own component then this component must be comp $([I])$. This can be proved by easy calculations and estimates.

We established the following inverse systems

$$
\cdots \longrightarrow \mathcal{U} \Psi^{0, k, s+1} \longrightarrow \mathcal{U} \Psi^{0, k, s} \longrightarrow \cdots \longrightarrow \mathcal{U} \Psi^{0, k, s_{0}}
$$

and

$$
\cdots \longrightarrow\left(\mathcal{U} \Psi^{0, k, s+1}\right)_{*} \longrightarrow\left(\mathcal{U} \Psi^{0, k, s}\right)_{*} \longrightarrow \cdots \longrightarrow\left(\mathcal{U} \Psi^{0, k, s_{0}}\right)_{*}
$$

with $\mathcal{U} \Psi^{0, k}=\lim _{\leftarrow} \mathcal{U} \Psi^{0, k, s}$ and $\left(\mathcal{U} \Psi^{0, k}\right)_{*}=\lim _{\leftarrow}\left(\mathcal{U} \Psi^{0, k, s}\right)_{*}$.

The tangent space $T_{[I]}\left(\mathcal{U} \Psi^{0, k, s}\right)_{*}$ can be described as follows:

$$
\begin{aligned}
& T_{[I]}\left(\mathcal{U} \Psi^{0, k, s}\right)_{*} \\
& =T_{[I]}(\operatorname{comp}([I]))_{*}=T_{[I]} \operatorname{comp}([I]) \\
& =T_{[I]}\left([I]+\left\{\left.[A] \in \mathcal{U} \Psi^{0, k, s}|| a_{0}\right|_{k+s} ^{2}+\left|a_{-1}\right|_{k-1+s}^{2}+\cdots+\left|a_{-k}\right|_{s}^{2}<\infty\right\}\right) \\
& =\left\{\left.[A] \in \mathcal{U} \Psi^{0, k, s}|| a_{0}\right|_{k+s} ^{2}+\left|a_{-1}\right|_{k-1+s}^{2}+\cdots+\left|a_{-k}\right|_{s}^{2}<\infty\right\} \\
& =\text { zero component of } \mathcal{U} \Psi^{0, k, s} \\
& \cong \Omega^{0,2, k+s}(S) \oplus \cdots \oplus \Omega^{0,2, s}(S) .
\end{aligned}
$$

This yields an inverse system

$$
\cdots \longrightarrow T_{[I]}\left(\mathcal{U} \Psi^{0, k, s+1}\right)_{*} \longrightarrow T_{[I]}\left(\mathcal{U} \Psi^{0, k, s}\right)_{*} \longrightarrow \cdots
$$

and

$$
\lim _{\leftarrow} T_{[I]}\left(\mathcal{U} \Psi^{0, k, s}\right)_{*}=\left\{[A] \in \mathcal{U} \Psi^{0, k} \mid a_{0}, a_{-1}, \ldots a_{-k} \in \Omega^{0,2, s}(S) \text { for all } s\right\} .
$$

We denote the latter space by $L \mathcal{U} \Psi^{0, k}:=\lim _{\leftarrow} T_{[I]}\left(\mathcal{U} \Psi^{0, k, s}\right)_{*}$. This is in fact a Lie algebra with respect to the bracket of $\Psi$ DOs.

Hence we proved the following 
Theorem 6.5. Assume $\left(M^{n}, g\right)$ open satisfying the conditions $(I)$ and $\left(B_{\infty}\right)$. Then $\left\{\left(\mathcal{U} \Psi^{0, k}\right)_{*},\left(\mathcal{U} \Psi^{0, k, s}\right)_{*} \mid s>n\right\}$ is an ILH Lie group and each $\left(\mathcal{U} \Psi^{0, k, s}\right)_{*}$ is a smooth Hilbert Lie group. Its ILH Lie algebra is $\left\{L \mathcal{U} \Psi^{0, k}, T_{[I]}\left(\mathcal{U} \Psi^{0, k, s}\right)_{*} \mid s>n\right\}$. Here $L \mathcal{U} \Psi^{0, k}$ is isomorphic, as topological vector space, to $\sum_{1}^{k} \Omega^{0,2, \infty}(S)$, where $\Omega^{0,2, \infty}(S)=\bigcap_{s} \Omega^{0,2, s}(S)$.

Corollary 6.6. $\left(\mathcal{U} \Psi^{0}\right)_{*}$ has the structure of a direct limit of ILH Lie groups,

$$
\left(\mathcal{U} \Psi^{0}\right)_{*}=\underset{\vec{k}}{\lim }\left(\mathcal{U} \Psi^{0, k}\right)_{*} .
$$

\section{An ILH Lie group structure for invertible Fourier integral operators.}

We consider our exact sequence (5.24),

$$
I \longrightarrow\left(\mathcal{U} \Psi^{0, k}\right)_{*} \longrightarrow\left(\mathcal{U} F^{0, k}(\infty)\right)_{*} \longrightarrow \mathcal{D}_{\theta, 0}^{\infty} \longrightarrow e
$$

and perform the 4th steps of our program described after Lemma 5.9. We know already that $\left(\mathcal{U} \Psi^{0, k}\right)_{*}$ and $\mathcal{D}_{\theta, 0}^{\infty}$ are ILH Lie groups, in particular they are topological groups.

We first consider an exact sequence of abstract groups

$$
I \longrightarrow \mathcal{H} \stackrel{j}{\longrightarrow} \mathcal{G} \stackrel{\pi}{\longrightarrow} \mathcal{Q} \longrightarrow e
$$

We assume that $\mathcal{H}$ and $\mathcal{Q}$ are topological groups and construct a topology on $\mathcal{G}$ such that (7.1) becomes an exact sequence of topological groups. In a second step we will sharpen the construction to the case of ILH Lie groups. The frame work of this approach is given in Adams-Ratiu-Schmid [2]. We recall without proofs the facts established there and concentrate our attention to the new features coming from the openness of the underlying manifold $M$.

Lemma 7.1. Let

$$
I \longrightarrow H \stackrel{j}{\longrightarrow} G \stackrel{\pi}{\longrightarrow} Q \longrightarrow e
$$

be an exact sequence of groups where $H$ and $Q$ are topological groups. Let $U \subset Q$ be a neighborhood of the identity $e \in Q$ and $\sigma: U \longrightarrow G$ a local section of $\pi$; let $V \subset U$ be a neighborhood of $e \in Q$ such that $V \cdot V^{-1} \subset U$ and assume 
A) the map $V \times V \times H \longrightarrow H$ given by

$$
\left(f_{1}, f_{2}, h\right) \longrightarrow \sigma\left(f_{1}\right) \sigma\left(f_{2}\right)^{-1} h \sigma\left(f_{1} f_{2}^{-1}\right)^{-1}
$$

is continuous;

B) for each $g \in G$ and $W \subset U$ such that $\pi(g) W \pi(g)^{-1} \subset U$, the map $W \times H \longrightarrow H$ given by

$$
(f, h) \longrightarrow g h \sigma(f) g^{-1} \sigma\left(\pi(g) f \pi(g)^{-1}\right)^{-1}
$$

is continuous.

Then $G$ can be made into a topological group such that $j, \pi$ and $\sigma$ are continuous and $\pi$ is open.

Remark. If $Q$ is connected then the condition $A$ ) is already sufficient.

Assume now $\mathcal{H}$ and $\mathcal{Q}$ in (7.1) to be ILH Lie groups with $\mathcal{H}=\lim _{\leftarrow} H^{s}$ and $\mathcal{Q}=\lim _{\leftarrow} Q^{t}, s \geq s_{0}, t \geq t_{0}$. Denote by $\mathcal{H}^{s}$ and $\mathcal{Q}^{t}$ the spaces $\mathcal{H}$ and $\mathcal{Q}$ with the coarser topologies of $\mathcal{H}^{s}$ and $\mathcal{Q}^{t}$, respectively. Then $\mathcal{H}^{s}$ and $\mathcal{Q}^{t}$ are topological groups.

Suppose $U \subset \mathcal{Q}^{t_{0}}$ open (hence open in all $\mathcal{Q}^{t}$ ), $\sigma: U \longrightarrow \mathcal{G}$ a local section and $V \subset U$ open in $\mathcal{Q}^{t_{0}}$ such that $V \cdot V^{-1} \subset U$. Denote $V$ with the topology from $\mathcal{Q}^{t}$ by $V^{t}$. Assume that for each $t \geq t_{0}$ there exists an $s(t)$ such that

$\left.\mathrm{A}^{\prime}\right)$ The map $V^{t} \times V^{t} \times \mathcal{H}^{s(t)} \longrightarrow \mathcal{H}^{s(t)}$ defined by

$$
\left(f_{1}, f_{2}, h\right) \longrightarrow \sigma\left(f_{1}\right) \cdot \sigma\left(f_{2}\right)^{-1} h \sigma\left(f_{1} f_{2}^{-1}\right)^{-1}
$$

is continuous;

B') for each $g \in \mathcal{G}$ and $W \subset U$ with $\pi(g) W \pi(g)^{-1} \subset U$, the map $W^{t} \times$ $\mathcal{H}^{s(t)} \longrightarrow \mathcal{H}^{s(t)}$ defined by

$$
(f, h) \longrightarrow g h \sigma(f) g^{-1} \sigma\left(\pi(g) f \pi(g)^{-1}\right)^{-1}
$$

is continuous.

Then we derive from Lemma 7.1 that for each $t$ the group $\mathcal{G}$ becomes a topological group $\mathcal{G}^{t}$, and we obtain an exact sequence of topological groups for all $t \geq t_{0}$,

$$
I \longrightarrow \mathcal{H}^{s(t)} \stackrel{j}{\longrightarrow} \mathcal{G}^{t} \stackrel{\pi}{\longrightarrow} \mathcal{Q}^{t} \longrightarrow e
$$


Taking the inverse limit topology, we obtain (7.1) as a sequence of topological groups.

We recall the right uniform structure of a topological group $G$. A sequence $\left(x_{n}\right)_{n}$ is a Cauchy sequence with respect to this structure if for any neighborhood $V$ of the identity there exists an $n_{0}$ s. t. $x_{n} x_{m}^{-1} \in V$ for all $m, n \geq n_{0}$.

Lemma 7.2. Let $X$ be a locally Hilbert topological group. Then $X$ is complete in its right uniform structure.

Corollary 7.3. $\overline{\mathcal{H}^{s}}=H^{s}$ and $\overline{\mathcal{Q}^{t}}=Q^{t}$.

Proof. $\mathcal{H}^{s}$ is dense in $H^{s}, \mathcal{Q}^{t}$ is dense in $Q^{t}$. Then apply Lemma 7.2.

Let $G^{t}$ be the completion of $\mathcal{G}^{t}$ with respect to the right uniform structure. It is not yet clear that $G^{t}$ is a topological group or even a group.

Lemma 7.4. Let $\tilde{U}^{t_{0}} \subset Q^{t_{0}}$ be open and $\tilde{U}^{t_{0}} \cap \mathcal{Q}^{t_{0}}=U^{t_{0}}$. For $t \geq t_{0}$, let $\tilde{U}^{t}=\tilde{U}^{t_{0}} \cap Q^{t}$ and $\tilde{V}^{t_{0}} \subset \tilde{U}^{t_{0}}$ open with $\tilde{V}^{t_{0}} \cap \mathcal{Q}^{t_{0}}=V^{t_{0}}$ and $\tilde{V}^{t}=\tilde{V}^{t_{0}} \cap Q^{t}$, $t \geq t_{0}$. Assume that $\sigma: U \longrightarrow \mathcal{G}$ extends to a local section $\tilde{\sigma}: \tilde{U}^{t} \longrightarrow G^{t}$ and that

$\left.A^{\prime \prime}\right)$ The map $V^{t} \times V^{t} \times \mathcal{H}^{s(t)} \longrightarrow \mathcal{H}^{s(t)}$ from $A^{\prime}$ ) actually extends by $\tilde{\sigma}$ to a continuous map $\tilde{V}^{t} \times \tilde{V}^{t} \times H^{s(t)} \longrightarrow H^{s(t)}$.

Then $G^{t}$ is a topological group.

Next we want to endow $G^{t}$ with a manifold structure. Define $\Psi_{I}$ : $\pi^{-1}\left(U^{t}\right) \longrightarrow U^{t} \times H^{s(t)}$ by

$$
\Psi_{I}: g \longmapsto\left(\pi(g), g \sigma(\pi(g))^{-1}\right) .
$$

We try to consider this as a bundle chart and move this around on $G^{t}$ by right translations: Let $g_{0} \in G^{t}, q_{0}=\pi\left(g_{0}\right)$ and define on $\pi^{-1}\left(U q_{0}\right)$

$$
\Psi_{g_{0}}: \pi^{-1}\left(U^{t} q_{0}\right) \longrightarrow U^{t} q_{0} \times H^{s(t)}
$$

by

$$
\Psi_{g_{0}}: g \longmapsto\left(\pi(g), g g_{0}^{-1} \sigma\left(\pi(g) q_{0}^{-1}\right)^{-1}\right)
$$

To obtain an atlas, we need the transition condition that

$$
\Psi_{\tilde{g}_{0}} \circ \Psi_{g_{0}}^{-1}:\left(U^{t} q_{0} \cap U^{t} \tilde{q}_{0}\right) \times H^{s(t)} \longrightarrow\left(U^{t} q_{0} \cap U^{t} \tilde{q}_{0}\right) \times H^{s(t)}
$$


is $C^{k(t)}$. According to our definitions this is the map

$$
(f, h) \longmapsto\left(f, h \sigma\left(f q_{0}^{-1}\right) g_{0} \tilde{g}_{0}^{-1} \sigma\left(f \tilde{q}_{0}^{-1}\right)^{-1}\right) .
$$

Hence we add the condition

C) For $g_{0}, \tilde{g}_{0} \in G^{t}$ with $\pi\left(g_{0}\right)=q_{0}, \pi\left(\tilde{g}_{0}\right)=\tilde{q}_{0}$ assume that the map (7.6), (7.7) is $C^{k(t)}$, where $k(t)$ is an increasing function of $t$.

Finally to construct an ILH Lie group structure for $\mathcal{G}$ we need that multiplication $G^{r+k} \times G^{r} \longrightarrow G^{r}$ is $C^{k}$ for $k \leq k(r)$. As pointed out in [2], p. 30 , this leads to the final condition

D) Assume for $a \in G^{r+k}, b \in G^{r}$ with $\alpha=\pi(a), \beta=\pi(b)$ that the map

$$
\begin{gathered}
U^{r+k} \alpha \times U^{r} \beta \times H^{s(r)} \longrightarrow H^{s(r)}, \\
\left(f_{1}, f_{2}, h\right) \longmapsto \sigma\left(f_{1} \alpha^{-1} a h \sigma\left(f_{2} \beta^{-1}\right) a^{-1} \sigma\left(f_{1}, f_{2} \beta^{-1} \alpha^{-1}\right)^{-1}\right)
\end{gathered}
$$

is $C^{k}$ as long as $k \leq k(r)$.

Summarizing, we obtained

Theorem 7.5. Let

$$
I \longrightarrow \mathcal{H} \longrightarrow \mathcal{G} \longrightarrow \mathcal{Q} \longrightarrow e
$$

be an exact sequence of groups where $\mathcal{H}$ and $\mathcal{Q}$ have ILH Lie group structures. Suppose there exists a local section $\sigma: U \longrightarrow \mathcal{G}$ satisfying the conditions $A^{\prime \prime}$ ), $\left.B^{\prime}\right), C$ ) and $\left.D\right)$. Then $\mathcal{G}$ has an ILH Lie group structure and (7.1) becomes an exact sequence of ILH Lie groups. If $\mathcal{Q}$ is connected then the condition $B^{\prime}$ ) follows from $\left.A^{\prime \prime}\right)$.

For the proofs of 7.1-7.5 we refer to Adams-Ratiu-Schmid [2].

Now we apply Theorem 7.5 to our situation where $\mathcal{H}=\lim _{\leftarrow}\left\{H^{s} \mid s \geq s_{0}\right\}$ with $H^{s}=\left(\mathcal{U} \Psi^{0, k, s}\right)_{*}, s_{0}=n+1, \mathcal{Q}=\lim _{\leftarrow}\left\{Q^{t} \mid t \geq t_{0}\right\}$ with $Q^{t}=\mathcal{D}_{\theta, 0}^{t}$, $t_{0}=n+1$ and $\mathcal{G}=\left(\mathcal{U} F^{0, k}(\infty)\right)_{*}$,

$$
\begin{gathered}
I \longrightarrow\left(\mathcal{U} \Psi^{0, k}\right)_{*} \longrightarrow\left(\mathcal{U} F^{0, k}(\infty)\right)_{*} \longrightarrow \mathcal{D}_{\theta, 0}^{\infty}\left(T^{*} M \backslash 0\right) \longrightarrow e \\
\sigma: \mathcal{U} \subset \mathcal{D}_{\theta, 0}^{\infty}\left(T^{*} M \backslash 0\right) \longrightarrow\left(\mathcal{U} F^{0, k}(\infty)\right)_{*}
\end{gathered}
$$

defined by (5.60). We have to verify the conditions above.

As always now, we assume $\left(M^{n}, g\right)$ with $(I),\left(B_{\infty}\right)$ and

$$
\inf \sigma_{e}\left(\left.\triangle_{1}(S)\right|_{\text {ker } \left.\triangle_{1}(S)\right)^{\perp}}\right)>0 .
$$


Proposition 7.6. Let $\sigma: \mathcal{U} \subset \mathcal{D}_{\theta, 0}^{\infty}\left(T^{*} M \backslash 0\right) \longrightarrow(\mathcal{U} F 0, k(\infty))_{*}$ be defined by (5.60), $V \subset \mathcal{U}$ be a neighborhood of $e \in \mathcal{D}_{\theta, 0}^{\infty}$ such that $V \cdot V^{-1} \in \mathcal{U}$. Then the condition $A)$ is satisfied, i.e., the map

$$
\begin{gathered}
V \times V \times\left(\mathcal{U} \Psi^{0, k}\right)_{*} \longrightarrow\left(\mathcal{U} \Psi^{0, k}\right)_{*}, \\
\left(f_{1}, f_{2}, A\right) \longrightarrow \sigma\left(f_{1}\right) \sigma\left(f_{2}\right)^{-1} A \sigma\left(f_{1} f_{2}^{-1}\right)^{-1},
\end{gathered}
$$

is continuous and extends as $C^{r}$ map to certain Sobolev completions, which will be specified below.

Proof. We are done if we can prove the following fact. Let $A, B \in\left(\mathcal{U} F^{0, k}\right)_{*}$ near the identity. Then the symbols of $A \circ B$ and $A^{-1}$ depend continuously on the symbols and phase functions of $A$ and $B$, i. e. if $\varphi_{H_{1}}, H_{1}=\Psi\left(f_{1}\right)$, $\varphi_{H_{2}}, H_{2}=\Psi\left(f_{2}\right), \varphi_{H}, H=\Psi\left(f_{1} f_{2}\right), \varphi_{H^{-}}, H^{-}=\Psi\left(f_{1}^{-}\right)$, are global phase functions for $A, B, A \circ B, A^{-1}$, respectively, and $a, b, c, c^{-}$are global symbols, $A^{\prime}, B^{\prime}$ operators of the same kind with $\varphi_{H_{1}^{\prime}}, \varphi_{H_{2}^{\prime}}, \varphi_{H^{\prime}} \varphi_{H^{\prime-}}, a^{\prime}$ etc., then

$$
\left|H-H^{\prime}\right|_{\text {Sob }} \leq P_{1}\left(\left|H_{1}-H_{1}^{\prime}\right|_{\text {Sob }},\left|H_{2}-H_{2}^{\prime}\right|_{\text {Sob }}\right),
$$

$$
\left|H^{-}-H^{\prime-}\right|_{\text {Sob }} \leq P_{2}\left(\left|H_{1}-H_{1}^{\prime}\right|_{\text {Sob }}\right),
$$

$$
\begin{aligned}
& \left|c-c^{\prime}\right|_{\text {Sob }} \leq P_{3}\left(\left|a-a^{\prime}\right|_{\text {Sob }},\left|b-b^{\prime}\right|_{\text {Sob }},\left|H_{1}-H_{1}^{\prime}\right|_{\text {Sob }},\left|H_{2}-H_{2}^{\prime}\right|_{\text {Sob }}\right), \\
& \left|a^{-}-a^{\prime-}\right|_{\text {Sob }} \leq P_{4}\left(\left|a-a^{\prime}\right|_{\text {Sob }},\left|H_{1}-H_{1}^{\prime}\right|_{\text {Sob }}\right) .
\end{aligned}
$$

Here the $P_{j}$ are polynomials without constant terms and | | Sob means certain Sobolev norms, the Sobolev index of which remains still open for a moment. Cover $M$ by a uniformly locally finite cover $U=\left\{U_{i}\right\}_{i}$ of normal charts. Then it is a well known fact that there exist constants $C_{1}, C_{2}$ s. t.

$$
C_{1} \sum_{i}||_{\text {Sob }, U_{i}}^{2} \leq||_{\text {Sob }}^{2} \leq C_{2} \sum_{i}||_{\text {Sob }, U_{i}}^{2} .
$$

(7.14) immediately implies that we are done if we can show (7.10)-(7.13) locally, i.e.,

$$
\begin{aligned}
\left|H-H^{\prime}\right|_{S o b, U_{i}} & \leq C \cdot P_{1}\left(\left|H_{1}-H_{1}^{\prime}\right|_{\mathrm{Sob}, U_{i}},\left|H_{2}-H_{2}^{\prime}\right|_{\mathrm{Sob}, U_{i}}\right), \\
\left|H^{-}-H^{\prime-}\right|_{\mathrm{Sob}, U_{i}} & \leq C \cdot P_{2}\left(\left|H_{1}-H_{1}^{\prime}\right|_{\mathrm{Sob}, U_{i}}\right), \\
\left|c-c^{\prime}\right|_{\mathrm{Sob}, U_{i}} & \leq C \cdot P_{3}(\ldots) \\
\left|a^{-}-a^{\prime-}\right|_{\mathrm{Sob}, U_{i}} & \leq C \cdot P_{4}(\ldots) .
\end{aligned}
$$


with $C$ independent of $i$. This is more or less explicitly done in [2], Lemma 4.2 and 4.3 , pp. $32-35$. We recall the initial step. In local coordinates we can write

$$
\begin{aligned}
A u(x) & =(2 \pi)^{-n} \iint e^{i(x-y) \xi+H_{1}(x, \xi)} a(x, \xi) u(y) d y d \xi \\
B u(x) & =(2 \pi)^{-n} \iint e^{i(x-y) \xi+H_{2}(x, \xi)} b(x, \xi) u(y) d y d \xi \\
A \circ B u(x) & =(2 \pi)^{-n} \iint e^{i(x-y) \eta+H_{1}(x, \eta)+y \xi+H_{2}(y, \xi)} a(x, \eta) b(y, \eta) \hat{u}(\xi) d \xi d y d \eta \\
& =(2 \pi)^{-n} \iint e^{i(x \xi+H(x, \xi))} c(x, \xi) \hat{u}(\xi) d \xi
\end{aligned}
$$

with $H_{1}=\Psi\left(f_{1}\right), H_{2}=\Psi\left(f_{2}\right), H=\Psi\left(f_{1} \circ f_{2}\right)$. Hence

$$
c(x, \xi) \sim(2 \pi)^{-n} \iint e^{i \varphi(x, y, \xi, \eta)} a(x, \eta) b(y, \xi) d y d \eta
$$

with $\varphi(x, y, \xi, \eta)=(x-y) \cdot(\eta-\xi)+H_{1}(x, \eta)+H_{2}(y, \xi)-H(x, \xi)$. Locally we have for (7.17) to establish the continuity of the map

$$
\begin{gathered}
S^{0, k} \times S^{0, k} \times \mathcal{W} \times \mathcal{W} \longrightarrow S^{0, k} \\
\left(a(x, \xi), b(x, \xi), H_{1}, H_{2}\right) \longmapsto c(x, \xi)
\end{gathered}
$$

and its extension to a certain Sobolev completion,

$$
S^{0, k, \tilde{s}(t)} \times S^{0, k, \tilde{s}(t)} \times \mathcal{W}^{t+r} \times \mathcal{W}^{t+r} \longrightarrow S^{0, k, \tilde{s}(t)} .
$$

Here $S^{o, k}$ is defined by $\mathcal{U} S^{0} / \mathcal{U} S^{-k-1}, \mathcal{U} S^{q}=\mathcal{U} S^{q}(B) \equiv \mathcal{U} S^{q}\left(B \times \mathbb{R}^{n}\right)$. If we could establish the continuity of (7.20)-(7.22), then we would have (7.17) by difference constructions with the gotten formulas, if these formulas permit such a construction. This is in fact the case. We refer to [2], p. 34-35. The main point is to calculate or estimate (7.19). This has been done in [2] by the method of stationary phase (as one would expect). Finally the Sobolev index in (7.17) and (7.20) is $\tilde{s}(t)=2(t-k-1)$. The estimate (7.15) is trivial as we have see from the last representation for $A \circ B$. The same holds for (7.16) and (7.18) which follows from Lemma 4.3 in [2] after some calculations.

Now Lemma 7.1 and Proposition 7.6 imply the following 
Theorem 7.7. $\left(\mathcal{U} F^{0, k}(\infty)\right)_{*}$ is a topological group, (5.24) is an exact sequence of topological groups and the local section $\sigma$ is continuous.

We write in the sequel $\mathcal{U} F^{0, k}$ instead of $\mathcal{U} F^{0, k}(\infty)$ since we consider only that space. Now let $H^{s}=\left(\mathcal{U} \Psi^{0, k}\right)_{*}^{s}$ and $Q^{t}=\left(\mathcal{D}_{\theta, 0}^{\infty}\left(T^{*} M \backslash 0\right)\right)^{t}$ the space $\mathcal{D}_{\theta, 0}^{\infty}$ with coarser topology of $\mathcal{D}_{\theta, 0}^{t}\left(T^{*} M \backslash 0\right)$. Proposition 7.6 implies that for $t>2 n$ and $s(t)=t-2(k+1)>n$ the map

$$
\begin{gathered}
V^{t} \times V^{t} \times\left(\mathcal{U} \Psi^{0, k}\right)_{*}^{s(t)} \longrightarrow\left(\mathcal{U} \Psi^{0, k}\right)_{*}^{s(t)} \\
\left(f_{1}, f_{2}, A\right) \longmapsto \sigma\left(f_{1}\right) \sigma\left(f_{2}\right)^{-1} A \sigma\left(f_{1} f_{2}^{-1}\right)^{-1},
\end{gathered}
$$

is at least continuous. Hence we obtain from Proposition 7.1 that $\left(\mathcal{U} F^{o, k}\right)_{*}$ becomes a topological group $\left(\mathcal{U} F^{o, k}\right)_{*}^{t}$ s. t.

$$
I \longrightarrow\left(\mathcal{U} \Psi^{0, k}\right)_{*}^{s(t)} \stackrel{j}{\longrightarrow}\left(\mathcal{U} F^{0, k}\right)_{*}^{t} \stackrel{\pi}{\longrightarrow}\left(\mathcal{D}_{\theta, 0}^{\infty}\left(T^{*} M \backslash 0\right)\right)^{t} \longrightarrow e
$$

is an exact sequence of topological groups for $t \geq t_{0}=\max \{2 n, n+2(k+1)\}$. Let $\left(\mathcal{U} F^{0, k, t}\right)_{*}$ be the completion with respect to the right uniform structure. We will show that this is a topological group. For this we have to show that the local section $\sigma$ extends to a local section

$$
\tilde{\sigma}: \tilde{\mathcal{U}}^{t} \longrightarrow\left(\mathcal{U} F^{0, k, t}\right)_{*}
$$

and the map in condition $A^{\prime}$ ) extends to a continuous map $A^{\prime \prime}$ )

$$
\tilde{V}^{t} \times \tilde{V}^{t} \times\left(\mathcal{U} \Psi^{0, k, S(t)}\right)_{*} \longrightarrow\left(\mathcal{U} \Psi^{0, k, S(t)}\right)_{*} .
$$

Consider first the extension $\sigma \longrightarrow \tilde{\sigma}$. Let $f \in \tilde{\mathcal{U}}^{t}$. The $\tilde{\sigma}(f)$ should be an FIO of order 0 with symbol $a(x, \xi)=\sum_{j=0}^{k} a_{-j}(x, \xi)$ where $\left.\left(a_{-j}-a_{-j}^{\infty}\right)\right|_{S} \in$ $\Omega^{0,2, k+s(t)}(S)$ for a smooth $a_{-j}^{\infty} \in \mathcal{U} S^{-j}$ and phase function $\varphi_{h}$ generated by $H=\Psi(f)$ with $f \in \mathcal{D}_{\theta, 0}^{t}$ and $\left.H\right|_{S} \in \Omega^{0,2, k+1}(S)$. The definition (5.26) still makes sense as oscillatory integral of $a(x, \xi)$. The $H(x, \xi)$ can be differentiated enough times and $t>2 n$ will be sufficient for this. The continuity of the extension follows from the procedures in Proposition 7.6, i.e., we have the condition A") for $t>2 n$ and $s(t)>n$. Hence we have established

Theorem 7.8. Assume $t>2 n$ and $s(t)=t-2(k+1)>n$. Then

$$
I \longrightarrow\left(\mathcal{U} \Psi^{0, k, s(t)}\right)_{*} \stackrel{j}{\longrightarrow}\left(\mathcal{U} F^{0, k, t}\right)_{*} \stackrel{\pi}{\longrightarrow}\left(\mathcal{D}_{\theta}^{t}\left(T^{*} M \backslash 0\right)\right)^{t} \longrightarrow e
$$


is an exact sequence of topological groups such that $j$ and $\pi$ are continuous and $\pi$ is open. Moreover, (5.60) defines a continuous local section

$$
\tilde{\sigma}: \mathcal{U}^{t} \subset \mathcal{D}_{\theta, 0}^{t}\left(T^{*} M \backslash 0\right) \longrightarrow\left(\mathcal{U} F^{0, k, t}\right)_{*} .
$$

It remains to assure the conditions C) and D).

Lemma 7.9. Assume $t>2 n, s(t)=t-2(k+1)>n$. For $A_{0}, \tilde{A}_{0} \in$ $\left(\mathcal{U} F^{0, k, t}\right)_{*}$ let $\pi\left(A_{0}\right)=f_{0}, \pi\left(\tilde{A}_{0}\right)=\tilde{f}_{0}$. Then the condition $\left.C\right)$ is satisfied, i.e., the map

$$
\begin{gathered}
\mathcal{U}^{t} \cdot f_{0} \cap \mathcal{U}^{t} \cdot \tilde{f}_{0} \times\left(\mathcal{U} \Psi^{0, k, s(t)}\right)_{*} \longrightarrow\left(\mathcal{U} \Psi^{0, k, s(t)}\right)_{*}, \\
(f, A) \longmapsto A \sigma\left(f f_{0}^{-1}\right) A_{0} \tilde{A}_{0}^{-1} \sigma\left(f \tilde{f}_{0}^{-1}\right)^{-1}
\end{gathered}
$$

is of class $C^{t}$.

Proof. According to Theorem 6.5, multiplication in $\left(\mathcal{U} \Psi^{0, k, s}\right)_{*}$ is smooth. Hence it suffices to show that

$$
f \longmapsto \sigma\left(f f_{0}^{-1}\right) A_{0} \tilde{A}_{0}^{-1} \sigma\left(f \tilde{f}_{0}^{-1}\right)^{-1}
$$

is $C^{t}$. For this it is sufficient to show that the map

$$
f \longmapsto \sigma(f) A \sigma\left(f f_{A}\right)^{-1}
$$

is $C^{t}$ for $f$ and $f f_{A}$ near $i d$, where we have set $A=A_{0} \tilde{A}_{0}^{-1}, f_{A}=\pi(A)$. But this follows from Proposition 7.6.

Hence we have

Theorem 7.10. Assume $t>2 n, s(t)=t-2(k+1)>n$. Then $\left(\mathcal{U} F^{0, k, t}\right)_{*}$ is a Hilbert manifold of class $C^{t}$ modeled by $\Omega^{0,2, t+1}(S) \times\left(\mathcal{U} \Psi^{0, k, s(t)}\right)_{*}$

\section{Remarks.}

1. Charts in $\left(\mathcal{U} F^{0, k, t}\right)_{*}$ are defined by right translation of a chart at $I$. This implies automatically that right translation in $\left(\mathcal{U} F^{0, k, t}\right)_{*}$ is $C^{t}$.

2. As we have seen that $\left(\mathcal{U} \Psi^{0, k, s(t)}\right)_{*}$ consists of uncountable many components. The same holds then also for $j\left(\mathcal{U} \Psi^{0, k, s(t)}\right)_{*}$.

The last condition we have to verify is D). 
Lemma 7.11. Assume $t>2 n, s(t)=t-2(k+1)>n$. Then for $A \in$ $\left(\mathcal{U} F^{0, k, t+r}\right)_{*}, B \in\left(\mathcal{U} F^{0, k, t}\right)_{*}, f_{A}=\pi(A) f_{B}=\pi(B)$. Then the condition $D)$ is satisfied, i.e., the map

$$
\begin{gathered}
\mathcal{U}_{f_{A}}^{t+r} \times \mathcal{U}_{f_{B}}^{t+r} \times\left(\mathcal{U} \Psi^{0, k, s(t)}\right)_{*} \longrightarrow\left(\mathcal{U} \Psi^{0, k, s(t)}\right)_{*}, \\
\left(f_{1}, f_{2}, P\right) \longrightarrow \sigma\left(f_{1} f_{A}^{-1}\right) A P \sigma\left(f_{2}, f_{B}^{-1}\right) A^{-1} \sigma\left(f_{1} f_{2} f_{B}^{-1} f_{A}^{-1}\right.
\end{gathered}
$$

is of class $C^{l}$ for $l=\min \{r, t\}$.

Proof. We have that the map in condition A') (hence B') is $C^{t}$ and multiplication in $\mathcal{D}_{\theta, 0}^{\infty}\left(T^{*} M \backslash 0\right)$ is $C^{r}$ as a map $\mathcal{D}_{\theta, 0}^{t+r}\left(T^{*} M \backslash 0\right) \times \mathcal{D}_{\theta, 0}^{t}\left(T^{*} M \backslash 0\right) \longrightarrow$ $\mathcal{D}_{\theta, 0}^{t}\left(T^{*} M \backslash 0\right)$.

Summarizing, we state our final main result.

Theorem 7.12. Assume $\left(M^{n}, g\right)$ is an open Riemannian manifold satisfying the conditions $(I)$ and $\left(B_{\infty}\right)$ of bounded geometry and the condition

$$
\inf \sigma_{e}\left(\triangle_{1}\left(S\left(T^{*} M\right)\right),\left.g_{S}\right|_{\left(\operatorname{ker} \triangle_{1}\right)^{\perp}}\right)>0 .
$$

Then for any $k \in \mathbf{Z}_{+}$

1. $\left\{\mathcal{D}_{\theta, 0}^{\infty}\left(T^{*} M \backslash 0\right), \mathcal{D}_{\theta, 0}^{r}\left(T^{*} M \backslash 0\right) \mid r \geq n+1\right\}$ is an ILH Lie group.

2. $\left\{\left(\mathcal{U} \Psi^{0, k}\right)_{*},\left(\mathcal{U} \Psi^{0, k, s}\right)_{*} \mid s \geq n+1\right\}$ is an ILH Lie group and each $\left(\mathcal{U} \Psi^{0, k, s}\right)_{*}$ is a smooth Hilbert Lie group.

3. $\left\{\left(\mathcal{U} F^{0, k}\right)_{*},\left(\mathcal{U} F^{0, k, t}\right)_{*} \mid t>\max \{2 n, n+2(k+1)\}\right\}$ is an ILH Lie group with the following properties:

a. $\left(\mathcal{U} F^{0, k, t}\right)_{*}$ is a $C^{t}$ Hilbert manifold modeled on $\Omega^{0,2, t+1}\left(S\left(T^{*} M\right)\right) \times$ $\left(\mathcal{U} \Psi^{0, k, t-2(k+1)}\right)_{*}$. Each component of $\left(\mathcal{U} F^{0, k, t}\right)_{*}$ is modeled by $\Omega^{0,2, t+1}(S) \oplus \Omega^{0,2, k+t-2(k+1)}(S) \oplus \cdots \oplus \Omega^{0,2, t-2(k+1)}(S)$.

b. The inclusion $\left(\mathcal{U} F^{0, k, t+1}\right)_{*} \hookrightarrow\left(\mathcal{U} F^{0, k, t+1}\right)_{*}$ is $C^{t}$.

c. The group multiplication $\left(\mathcal{U} F^{0, k}\right)_{*} \times\left(\mathcal{U} F^{0, k}\right)_{*} \longrightarrow\left(\mathcal{U} F^{0, k}\right)_{*}$ extends to a $C^{l}$ map

$$
\left(\mathcal{U} F^{0, k, t+r}\right)_{*} \times\left(\mathcal{U} F^{0, k, t}\right)_{*} \longrightarrow\left(\mathcal{U} F^{0, k, t}\right)_{*},
$$

$l=\min \{r, t\}$. 
d. The inversion $\left(\mathcal{U} F^{0, k}\right)_{*} \longrightarrow\left(\mathcal{U} F^{0, k}\right)_{*}$ extends to a $C^{l}$ map

$$
\left(\mathcal{U} F^{0, k, t+r}\right)_{*} \longrightarrow\left(\mathcal{U} F^{0, k, t}\right)_{*},
$$

$$
l=\min \{r, t\} .
$$

e. Right multiplication for $A \in\left(\mathcal{U} F^{0, k, t}\right)_{*}$ is a $C^{t}$ map $R_{A}$ : $\left(\mathcal{U} F^{0, k, t}\right)_{*} \longrightarrow\left(\mathcal{U} F^{0, k, t}\right)_{*}$

We finish with a few remarks concerning the corresponding Lie algebras with differ slightly from the statements in [2]. First we present the version of [2], 5.2 for the open case.

Lemma 7.13. The Lie algebra of $\left(\mathcal{U} F^{0, k, s}\right)_{*}$ is $\operatorname{Comp}_{0} \widetilde{\mathcal{U}}^{1, k, s}$, the 0component of the completed space $\widetilde{\mathcal{U} \Psi}^{1, k, s}$ of formal pseudodifferential operators of order one modulo those of order $-k-1$ with pure imaginary principal symbol. The Lie bracket corresponds to the commutator bracket.

Proof. Let $c(t)$ be a $C^{1}$ curve in $\left(\mathcal{U} F^{0, k, s}\right)_{*}$ with $c(0)=I$. We can write $c(t)=P(t) \sigma(\pi(c(t)))$, where $P(t)$ is a $C^{1}$ curve in $\mathcal{U} \Psi^{1, k, s}$ such that $P(0)=I$ and $\sigma(\pi(c(t)))$ has the local expression

$$
\sigma(\pi(c(t))) u(x)=(2 \pi)^{-n} \iint e^{i\left(\varphi_{0}(x, y, \xi)+H_{1}(x, \xi)\right)} u(y) d y d \xi
$$

with $\varphi(x, y, \xi)=\langle x-y, \xi\rangle, H_{0}(x, \xi)=0,\left.H_{1}(x, \xi)\right|_{S} \in \Omega^{0,2, k+s+1}(S)$. Differentiation at $t=0$ yields $c^{\prime}(0)=P^{\prime}(0)+A$, where locally

$$
A u(x)=(2 \pi)^{-n} \iint e^{i \varphi_{0}(x, y, \xi)} i H_{0}^{\prime}(x, \xi) u(y) d y d \xi .
$$

$H_{t}(x, \xi)$ is homogeneous of degree one in $\xi$ for each $t$. Hence the same holds for $H_{0}^{\prime}(x, \xi), H_{0}^{\prime} \in i \cdot \mathcal{H}^{1} \Omega^{0,2, k+s+1}\left(T^{*} M \backslash 0\right)$. This implies $A \in i$. $\operatorname{Comp}_{0} \widetilde{\mathcal{U} \Psi}^{1, k, s}$. Moreover, as we have seen in section 6 after 6.4 that $P^{\prime}(0) \in$ $C o m p_{0} \mathcal{U} \Psi^{0, k, s}$, so $c^{\prime}(0) \in C o m p_{0} \widetilde{\mathcal{U}}^{1, k, s}$. It is easy to see that the map $c \mapsto c^{\prime}(0)$ is surjective and that the Lie bracket is the commutator bracket.

Hence we have 
Theorem 7.14. The exact sequence of ILH Lie groups

$$
I \longrightarrow\left(\mathcal{U} \Psi^{0, k, s}\right)_{*} \longrightarrow\left(\mathcal{U} F^{0, k, s}\right)_{*} \longrightarrow \mathcal{D}_{\theta, 0}^{s+k}\left(T^{*} M \backslash 0\right) \longrightarrow e
$$

has as corresponding sequence of ILH Lie algebras

(7.23) $0 \longrightarrow \operatorname{Comp}_{0} \mathcal{U} \Psi^{0, k, s} \longrightarrow \operatorname{Comp}_{0} \widetilde{\mathcal{U} \Psi}^{1, k, s}$

$$
\stackrel{\varrho}{\longrightarrow} \mathcal{H}^{1} \Omega^{0,2, s+k+1}\left(T^{*} M \backslash 0\right) \longrightarrow 0,
$$

where $\varrho=\frac{1}{i} \times$ principal symbol.

\section{Remarks.}

1. As exact sequence of vector spaces (7.23) corresponds to

$$
\begin{aligned}
0 \longrightarrow \sum_{i=0}^{k} \Omega^{0,2, i+s}(S) \longrightarrow\left(\sum_{i=0}^{k} \Omega^{0,2, i+s}(S)\right) \oplus & \Omega^{0,2, k+s+1}(S) \\
& \longrightarrow \Omega^{0,2, k+s+1}(S) \longrightarrow 0 .
\end{aligned}
$$

2. The space $\mathcal{M}^{\infty}\left(I, B_{\infty}\right)=\lim \mathcal{M}^{r}\left(I, B_{\infty}\right)$ splits into an uncountable $\overleftarrow{r}$

number of components.

$$
\mathcal{M}^{\infty}\left(I, B_{\infty}\right)=\sum_{i \in I} \operatorname{comp}\left(g_{i}\right)
$$

where

$$
\begin{aligned}
\operatorname{comp}(g)= & \left\{g^{\prime} \mid g^{\prime} \text { satisfies }(I) \text { and }\left(B_{\infty}\right), g \text { and } g^{\prime}\right. \\
& \text { are quasiisometric and }\left|g-g^{\prime}\right|_{g, r} \\
& :=\left(\int\left(\left|g-g^{\prime}\right|_{g, x}^{2}+\sum_{i=0}^{r-1}\left|\left(\nabla^{g}\right)^{i}\left(\nabla^{g}-\nabla^{g^{\prime}}\right)\right|_{g, x}^{2}\right) d v o l_{x}(g)\right)^{\frac{1}{2}} \\
& <\infty \text { for all } r\} .
\end{aligned}
$$

$g^{\prime} \in \operatorname{comp}(g)$ implies that $\left(S_{g}\left(T^{*} M\right), g_{S}\right)$ and $\left(S_{g^{\prime}}\left(T^{*} M\right), g_{S}^{\prime}\right)$, are quasiisometric and $g_{S}^{\prime} \in \operatorname{comp}\left(g_{S}\right)$. Moreover, all functional spaces which we considered and which enter into the construction of $\mathcal{U} \Psi^{0, k, s}, \mathcal{U} F^{0, k, s}, \mathcal{D}^{s} S_{\theta, 0}$ are invariants of $\operatorname{comp}(g)$ like the initial spaces $\mathcal{U} \Psi^{q}, \mathcal{U} F^{q}$. We obtain that the ILH Lie group structure of $\mathcal{U} F^{0, k}$ is an invariant of comp $(g)$. We refer to [8] where we constructed the spaces $\mathcal{M}^{r}\left(I, B_{k}\right)$. 


\section{References.}

[1] M.R. Adams, T. Ratiu and R. Schmid, A Lie Group Structure for Pseudodifferential Operators, Math. Ann., 273 (1986), 529-551.

[2] M.R. Adams, T. Ratiu and R. Schmid, A Lie Group Structure for Fourier Integral Operators, Math. Ann., 276 (1986), 19-41.

[3] M.R. Adams, T. Ratiu and R. Schmid, The Lie group structure of diffeomorphism groups and invertible Fourier integral operators, with applications, MSRI Publications, 4, V. Kac (Ed), (1985), 1-69.

[4] M. Adler, On a trace functional for formal pseudo-differential operators and the symplectic structure for the Korteweg-de Vries type equations, Invent. Math., 50 (1979), 219-248.

[5] D.E. Blair, Contact Manifolds in Riemannian Geometry, Lecture Notes in Mathematics, 509, Berlin, 1976.

[6] D.E. Blair, On the set of metrics associated to a symplectic or contact form, Bull. Inst. Math. Academica Sinica, 11(2) (1983), 297-308.

[7] J. Cheeger, M. Gromov M. Taylor, Finite propagation speed, kernel estimates for functions of the Laplace operator, and the geometry of complete Riemannian manifolds, J. Diff. Geom., 17 (1982), 15-53.

[8] J. Eichhorn, Spaces of Riemannian metrics on open manifolds, Results in Mathematics, 27 (1995), 256-283.

[9] J. Eichhorn, Elliptic operators on noncompact manifolds, TeubnerTexte Math., 106 (1988), 4-169.

[10] J. Eichhorn, The manifold structure of maps between open manifolds, Ann. of Global Analysis and Geometry, 11 (1993), 253-300.

[11] J. Eichhorn, Gauge theory on open manifolds of bounded geometry, Int. Journ. Mod. Physics, 7 (1993), 3927-3977.

[12] J. Eichhorn and J. Fricke, The Module Structure Theorem for Sobolev Spaces on Open Manifolds, Math. Nachr., 195 (1998).

[13] J. Eichhorn and J. Fricke, Important diffeomorphism groups on open manifolds, in preparation. 
[14] J. Eichhorn and Yu. Kordjukov, Differential operators with Sobolev coefficients, in preparation.

[15] J. Eichhorn and R. Schmid, Form preserving diffeomorphism groups on open manifolds, Ann. Global Analysis and Geometry, 14 (1996), $147-176$.

[16] Yu. Kordjukov, $L_{p}$-Theory of Elliptic Differential Operators on Manifolds of Bounded Geometry, Acta Applicandae Mathematicae, 23 (1991), 223-260.

[17] H. Omori, Infinite dimensional Lie transformation groups, Lecture Notes in Mathematics, 427, Berlin, 1974.

[18] T. Ratiu and R. Schmid, The differential structure of three remarkable diffeomorphism groups, Math. Z., 177 (1981), 81-100.

[19] R. Schmid, Infinite Dimensional Hamiltonian Systems, Monographs and Textbooks in Physical Sciences, Biblionopolis, Napoli, 1987.

[20] M.A. Shubin, Weak Block property and weight estimates for elliptic operators, Seminaire Equations aux Derivees Partielles, Ecole Polytechnique, Centre de Mathematiques, 1989-1990.

[21] A. Weinstein, Lectures on symplectic manifolds, CBMS Conference Series, 29, AMS, Providence, 1977.

INSTITUT FÜR MATHEMATIK

UNIVERSITÄT GREIFSWALD

JAHNSTR. 15A

D-17487 GREIFSWALD

GERMANY

E-mail address: eichhorn@rz.uni-greifswald.de

Department of Mathematics

EMORY UNIVERSITY

Atlanta, Georgia 30322

E-mail address: rudolf@mathcs.emory.edu

Received June 9, 1999. 